\title{
Time-dependent density-functional theory for extended systems
}

\author{
Silvana Botti ${ }^{1,2}$, Arno Schindlmayr ${ }^{1,3,4}$, Rodolfo Del Sole ${ }^{1,5}$ and \\ Lucia Reining ${ }^{1,2}$ \\ ${ }^{1}$ European Theoretical Spectroscopy Facility (ETSF) \\ ${ }^{2}$ Laboratoire des Solides Irradiés, CNRS-CEA, École Polytechnique, 91128 Palaiseau, \\ France \\ ${ }^{3}$ Institut für Festkörperforschung, Forschungszentrum Jülich, 52425 Jülich, Germany \\ ${ }^{4}$ Fritz-Haber-Institut der Max-Planck-Gesellschaft, Faradayweg 4-6, 14195 \\ Berlin-Dahlem, Germany \\ ${ }^{5}$ CNR-INFM Institute for Statistical Mechanics and Complexity, and Dipartimento di \\ Fisica dell'Università di Roma "Tor Vergata", 00133 Roma, Italy \\ E-mail: silvana.botti@polytechnique.fr
}

\begin{abstract}
For the calculation of neutral excitations, time-dependent density functional theory (TDDFT) is an exact reformulation of the many-body time-dependent Schrödinger equation, based on the knowledge of the density instead of the many-body wavefunction. The density can be determined in an efficient scheme by solving one-particle noninteracting Schrödinger equations - the Kohn-Sham equations. The complication of the problem is hidden in the -unknown- time-dependent exchange and correlation potential that appears in the Kohn-Sham equations and for which it is essential to find good approximations. Many approximations have been suggested and tested for finite systems, where even the very simple adiabatic local density approximation (ALDA) has often proved to be successful. In the case of solids, ALDA fails to reproduce optical absorption spectra, that are instead well described by solving the Bethe-Salpeter equation of manybody perturbation theory (MBPT). On the other hand, ALDA can lead to excellent results for loss functions (at vanishing and finite momentum transfer). In view of that and thanks to recent successful developments of improved linear response kernels derived from MBPT, TDDFT is today considered a promising alternative to MBPT for the calculation of electronic spectra, even for solids. After reviewing the fundamentals of TDDFT within linear response, we discuss different approaches and a variety of applications to extended systems.
\end{abstract}

Submitted to: Rep. Prog. Phys.

PACS numbers: 71.15.Mb, 71.10.-w, 71.35.Cc, 71.45.Gm 


\section{Introduction}

Most of today's quantum mechanical theoretical research in condensed matter physics and chemistry is not aimed at finding new fundamental interactions or basic laws - it deals with solving the Schrödinger equation of a well-known Hamiltonian, and extracting useful information from the solution. This Hamiltonian, however, describes a many-body problem, and for a number of electrons well above 10 it is impossible to even dream of a full numerically exact solution. Moreover the exact solution would yield a wealth of information that could hardly be understood without further analysis and simplification, and contain many details that, for a given situation or question, one is probably not interested in [1]. Therefore it is often more appropriate to reformulate the problem from the start, working with effective Hamiltonians or selected expectation values that are suitable for the solution of a reduced problem. This procedure will ideally simplify both the calculation and the analysis of the desired quantities.

Density-Functional Theory (DFT) [2] is a prominent example of such an approach. It has been designed for the calculation of ground-state properties. It is based on knowledge of the density $n(\mathbf{r})$ instead of the full many-body wavefunction $\Psi\left(\mathbf{r}_{1}, \mathbf{r}_{2}, \ldots ., \mathbf{r}_{N}, \sigma_{1}, \sigma_{2}, \ldots ., \sigma_{N}\right)$ of the $N$-particle system. DFT can be formulated in the Kohn-Sham (KS) approach [3] where an efficient one-particle non-interacting Schrödinger equation - the Kohn-Sham equation yields eigenvalues $\varepsilon_{i}$ and orbitals $\varphi_{i}(\mathbf{r})$. The orbitals and eigenvalues do in general not have a direct physical meaning, but the former can be used to construct the true density of the interacting system according to $n(\mathbf{r})=\sum_{i}\left|\varphi_{i}(\mathbf{r})\right|^{2}$. The complication of the problem is hidden in the - unknown- exchange and correlation (xc) total energy $E_{\mathrm{xc}}$ and the exchangecorrelation potential $v_{\mathrm{xc}}[n](\mathbf{r})$ that appears in the Kohn-Sham equation. Very efficient approximations have been proposed, such as the Local Density Approximation (LDA) [3] or Generalised Gradient Approximations (GGA) [4, 5], and many ground state properties are today calculated from first principles with a precision of a few percent, like lattice parameters or phonon frequencies [6]. There exist, however, ground state properties for which even in simple systems standard approximations do less well: cohesive energies in particular can easily be off by $10 \%$ in LDA (because of errors in calculating the isolated atoms that enter this total energy difference), and failures are reported for static response properties, like the dielectric constant $\epsilon_{\infty}$, which is often substantially overestimated [7]. Other problems arise e.g. in the description of strongly correlated systems [8], or of the van der Waals dispersion attraction [9]. These problems in calculating ground state properties can be traced back to limits of validity of the employed approximations.

Another problem of static ground state DFT-Kohn-Sham is the fact that excitations, such as those measured in the optical response to a time-dependent electric field, are in principle not accessible. This is not a question of the available approximations, but of the fact that the theory is not meant to describe these phenomena. In fact, even if one could calculate the exact Kohn-Sham eigenvalues, their differences would not necessarily be close to measured excitation energies. By definition, they do not stand for electron addition or removal energies either [10]. The fact that the Kohn-Sham gap is in general reported to be too small with respect to measured gaps does hence a priori not tell us anything about the quality of a chosen approximation for the exchange-correlation potential.

If one wishes to work with an efficient Hamiltonian that in principle yields eigenvalues meant to be electron addition or removal energies, or excitation energies, more than just the static ground state density has to be calculated. Such energies can be found in essentially two ways.

One, by studying particle propagation and fluctuations in the system. This yields 
correlation functions that can then be related to response functions yielding e.g. linear response for optical absorption. These correlation functions are one-or two body Green's functions [11] (or higher order ones, for problems beyond the scope of this review). The one-body Green's function (that can essentially be understood as a time-dependent particle and hole density matrix) has phase fluctuations (or, in frequency $(\omega-)$ space, poles) given by electron addition and removal energies, measured e.g. in photoemission or inverse photoemission experiments. The particle-hole part of the two-particle Green's function, in turn, has poles at the energies of neutral excitations. A contraction of the four-point reducible part $L\left(\mathbf{r}, \mathbf{r}_{1}, \mathbf{r}^{\prime}, \mathbf{r}_{1}^{\prime}, \omega\right)$ of the two-particle Green's function leads to the two-point response functions $\chi\left(\mathbf{r}, \mathbf{r}^{\prime}, \omega\right)$ that determine measurable spectra, like absorption or electron energy loss spectra (EELS). Many-body perturbation Theory (MBPT) yields a framework where suitable approximations for these Green's functions can be found; in particular the Bethe-Salpeter Equation (BSE) is a good starting point for approximations for $\chi[11,12,13,14]$. The price to be payed for a physically intuitive and in general quite reliable description is however relatively high in terms of computational cost, because now quantities like $L\left(\mathbf{r}, \mathbf{r}_{1}, \mathbf{r}^{\prime}, \mathbf{r}_{1}^{\prime}, \omega\right)$ appear, instead of the density $n(\mathbf{r})$.

Second, by actually (on paper or in the computer) exposing the system to a timedependent external potential and calculating the evolution of the density in time. The response function $\chi$, for example, can then directly be determined from the linear response relation $\left.n^{(1)}(\mathbf{r}, \omega)=\int d^{3} r^{\prime} \chi\left(\mathbf{r}, \mathbf{r}^{\prime}, \omega\right) v_{\text {ext }}^{(1)} \mathbf{r}^{\prime}, \omega\right)$ between the variation in the external potential and the induced density. This route has become accessible thanks to the extension of DFT to its time-dependent generalisation, TD-DFT $[15,16,17,18,19]$. Put on a rigorous basis by the Runge-Gross theorem [16], one can understand that in TDDFT the quantum-mechanical "trajectory" of the system under the influence of a time-dependent external potential is found by searching for the extrema of an action (instead of the minimisation of a total energy, as done for the ground state), by analogy to the case of classical mechanics. One obtains hence the time-dependent Kohn-Sham equations as generalisation of the static case, and from these, response functions describing neutral excitations of a system [20]. At this point the difficulty resides in finding suitable approximations for the time-dependent exchangecorrelation potential $v_{\mathrm{xc}}[n](\mathbf{r}, t)$; note that now the functional dependence is on the density in the whole space and at all past times.

Many approximations have been suggested and tested for finite systems. Even the very simple adiabatic local density approximation (ALDA, also called Time-Dependent LDA (TDLDA)) where $v_{\mathrm{xc}}^{A L D A}[n](\mathbf{r}, t)=v_{\mathrm{xc}}^{L D A}(n(\mathbf{r}, t))$ has been proved to be very successful in many cases $[15,21]$, although the lack of a long range $(1 / r)$ decay of the potential can lead to serious problems for questions like Rydberg states [22]. The latter shortcoming of the LDA potential is not so crucial in solids where the electron density is quite homogeneous (compared to an atom in empty space); instead, the wrong long-range behaviour of the linear response kernel $f_{\mathrm{xc}}\left(\mathbf{r}, \mathbf{r}^{\prime}, t, t^{\prime}\right)=\delta v_{\mathrm{xc}}[n](\mathbf{r}, t) / \delta n\left(\mathbf{r}^{\prime}, t^{\prime}\right)$ can cause serious problems [14]. In fact in the ALDA this kernel is proportional to $\delta\left(\mathbf{r}-\mathbf{r}^{\prime}\right)$, whereas in non-metallic systems it should decay as $1 /\left|\mathbf{r}-\mathbf{r}^{\prime}\right|$ [23]. This shortcoming already shows up, e.g., in the calculation of polarizabilities for molecular chains [24]. In the case of absorption spectra of solids, where the imaginary part of the dielectric function $\epsilon$ for vanishing wavevector $\mathbf{q}$ (corresponding to a macroscopic average) is calculated, the lack of a $1 / q^{2}$ divergence (stemming from the Fourier transform of $1 /\left|\mathbf{r}-\mathbf{r}^{\prime}\right|$ ) can lead to drastic failures. For example, the ALDA is not able to reproduce bound excitons [14]. On the other hand, for finite momentum transfer or when the loss function $-\operatorname{Im}\left(\epsilon^{-1}\right)$ is the quantity of interest (e.g. in Electron Energy Loss or Inelastic X-ray Scattering spectra) this term is not dominant, and ALDA can lead to very good results (see e.g. [25, 26, 27, 28]). For this reason, and because of recent successful developments of 
improved linear response kernels derived from MBPT [29, 30, 31, 32, 33, 34, 35], TDDFT is today considered to be a promising alternative to MBPT for the calculation of electronic spectra, even for solids.

This situation is the main motivation for summarising here the state-of-the art of the TDFFT approach for extended systems. This review is centred on the calculation of linear response, which actually constitutes the overwhelming majority of work done in the field. Moreover, it is limited to the calculation of excitation of valence electrons. TDDFT is also used to determine core electron spectra (see e.g. [36]); however, this case is in many respects more similar to the situation in finite systems and will therefore not be treated here. Complementary to the present review, a discussion of time-dependent current density functional theory for periodic systems can be found in Ref. [37]. A comprehensive review of many aspects of TDDFT can is Ref. [38].

After a brief review of the fundamentals of TDFFT for which several more detailed reviews can be consulted (see e.g. [19, 37, 21]), we will concentrate on questions that are more specific to extended systems, with a short comparison to finite systems. Rather than giving an exhaustive summary of all results that have been obtained in this rapidly expanding field, selected applications are presented to illustrate major points, such as the importance of crystal local field effects or the effect of contributions derived from MBPT. A short outlook follows the conclusions.

\section{Fundamentals}

As the quantum-mechanical treatment of stationary and time-dependent systems differs in many aspects, it is not straightforward to generalize the mathematical framework of static density-functional theory. For example, the total energy, which plays a central role in the original Hohenberg-Kohn theorem [2], is not a conserved quantity in the presence of timedependent external fields, and there is hence no variational principle for it on the basis of the density that can be exploited. In this section we start by discussing the theoretical foundations of TDDFT with a special emphasis on the linear density-response function and its connection to the electronic excitation spectrum. We will use atomic units throughout the paper (i.e. $e^{2}=\hbar=m_{\mathrm{e}}=1$ ).

\subsection{The Runge-Gross theorem}

The evolution of a (non-relativistic) spin-unpolarized interacting many-electron system is governed by the time-dependent Schrödinger equation

$$
\mathrm{i} \frac{\partial}{\partial t} \Psi(\{\mathbf{r}\}, t)=\hat{H}(\{\mathbf{r}\}, t) \Psi(\{\mathbf{r}\}, t), \quad \Psi\left(\{\mathbf{r}\}, t_{0}\right) \text { given },
$$

where $\hat{H}$ is the Hamiltonian operator of the system and $\{\mathbf{r}\}=\left\{\mathbf{r}_{1}, \ldots, \mathbf{r}_{N}\right\}$ are the spatial coordinates of the $N$ electrons. The Hamiltonian can be written in the form

$$
\hat{H}(\{\mathbf{r}\}, t)=\sum_{i=1}^{N}\left(-\frac{1}{2} \nabla_{i}^{2}+v_{\mathrm{ext}}\left(\mathbf{r}_{i}, t\right)\right)+\frac{1}{2} \sum_{i \neq j}^{N} v\left(\mathbf{r}_{i}-\mathbf{r}_{j}\right),
$$

where $v_{\text {ext }}(\mathbf{r}, t)$ is the time-dependent external potential and $v\left(\mathbf{r}_{i}-\mathbf{r}_{j}\right)=1 /\left|\mathbf{r}_{i}-\mathbf{r}_{j}\right|$ the Coulomb interaction. Being interested in spectroscopy, we consider scenarios where the system is initially at rest in a static potential $v_{\text {ext }}(\mathbf{r}, t)=v_{\text {ext }}^{0}(\mathbf{r})$, before a time-dependent perturbation is switched on at $t=t_{0}$ in order to probe the response of the electron system. 
Under these circumstances the initial state at $t_{0}$ is given by the stationary ground-state wave function $\Psi\left(\{\mathbf{r}\}, t_{0}\right)=\Psi_{0}(\{\mathbf{r}\}) \exp \left(-\mathrm{i} E_{0} t_{0}\right)$, where $E_{0}$ denotes the ground-state energy. This initial wave function is determined up to an irrelevant phase factor for non-degenerate systems. By virtue of the Hohenberg-Kohn theorem it is also a functional of the static ground-state density $n\left(\mathbf{r}, t_{0}\right)=n_{\mathrm{GS}}(\mathbf{r})$. We only admit physical potentials that are finite everywhere and vary smoothly in time, so that they can be expanded into a Taylor series about the initial time $t_{0}$

$$
v_{\text {ext }}(\mathbf{r}, t)=\sum_{k=0}^{\infty} \frac{c_{k}(\mathbf{r})}{k !}\left(t-t_{0}\right)^{k} \quad \text { with } \quad c_{k}(\mathbf{r})=\left.\frac{\partial^{k}}{\partial t^{k}} v_{\text {ext }}(\mathbf{r}, t)\right|_{t=t_{0}}
$$

The theoretical basis of TDDFT is the Runge-Gross theorem [16], which asserts the one-toone correspondence between the external potential and the density, thus playing the same role as the Hohenberg-Kohn theorem in static density-functional theory. Of course, for a given external potential it is always possible, in principle, to solve the time-dependent Schrödinger equation (2.1); the density is then given by

$$
n(\mathbf{r}, t)=N \int d^{3} r_{2} \int d^{3} r_{3} \ldots \int d^{3} r_{N}\left|\Psi\left(\mathbf{r}, \mathbf{r}_{2}, \ldots, \mathbf{r}_{N}, t\right)\right|^{2} .
$$

What remains to be proved, in order to demonstrate the one-to-one correspondence, is that if two potentials $v_{\text {ext }}(\mathbf{r}, t)$ and $v_{\text {ext }}^{\prime}(\mathbf{r}, t)$ differ by more than a purely time-dependent function, then the associated densities $n(\mathbf{r}, t)$ and $n^{\prime}(\mathbf{r}, t)$ must be distinct. The addition of a purely time-dependent function is exempt because it only changes the phase of the wave function but not the density. One assumes that both systems evolve from the same initial groundstate wave function $\Psi\left(\{\mathbf{r}\}, t_{0}\right)$. The expansion coefficients of the two potentials around $t_{0}$ are denoted by $c_{k}(\mathbf{r})$ and $c_{k}^{\prime}(\mathbf{r})$, and one defines $u_{k}(\mathbf{r})=c_{k}(\mathbf{r})-c_{k}^{\prime}(\mathbf{r})$. If the potentials differ by more than a purely time-dependent function, then at least one coefficient $u_{k}(\mathbf{r})$ is not a mere constant but a spatially varying function. For the proof of the Runge-Gross theorem given in [16] one makes use of the current density

$$
\begin{aligned}
\mathbf{j}(\mathbf{r}, t)=-\frac{\mathrm{i}}{2} N \int d^{3} r_{2} \int d^{3} r_{3} \ldots \int d^{3} r_{N} & \left\{\Psi^{*}\left(\mathbf{r}, \mathbf{r}_{2}, \ldots, \mathbf{r}_{N}, t\right) \nabla \Psi\left(\mathbf{r}, \mathbf{r}_{2}, \ldots, \mathbf{r}_{N}, t\right)\right. \\
& \left.-\nabla \Psi^{*}\left(\mathbf{r}, \mathbf{r}_{2}, \ldots, \mathbf{r}_{N}, t\right) \Psi\left(\mathbf{r}, \mathbf{r}_{2}, \ldots, \mathbf{r}_{N}, t\right)\right\}
\end{aligned}
$$

which can also be written in a second quantization formalism as

$$
\mathbf{j}(\mathbf{r}, t)=-\frac{\mathrm{i}}{2}\left\langle\Psi(t)\left|\hat{\psi}^{\dagger}(\mathbf{r})[\nabla \hat{\psi}(\mathbf{r})]-\left[\nabla \hat{\psi}^{\dagger}(\mathbf{r})\right] \hat{\psi}(\mathbf{r})\right| \Psi(t)\right\rangle
$$

The time evolution of the current density can be discussed by means of the equation of motion

$$
\mathrm{i} \frac{d}{d t} \mathbf{j}(\mathbf{r}, t)=\langle\Psi(t)|[\hat{\mathbf{j}}(\mathbf{r}), \hat{H}(t)]| \Psi(t)\rangle .
$$

Moreover, $\mathbf{j}(\mathbf{r}, t)$ is related to the density through the continuity equation

$$
\frac{\partial}{\partial t} n(\mathbf{r}, t)=-\nabla \cdot \mathbf{j}(\mathbf{r}, t)
$$

This identity expresses the conservation of the total particle number in a differential form: the change in the number of electrons within a certain volume equals the flux through its 
surface. In the first step one shows that the current densities $\mathbf{j}(\mathbf{r}, t)$ and $\mathbf{j}^{\prime}(\mathbf{r}, t)$ induced by the two potentials differ. To this effect one examines the time derivative

$$
\begin{aligned}
\mathrm{i} \frac{d}{d t}\left\{\mathbf{j}(\mathbf{r}, t)-\mathbf{j}^{\prime}(\mathbf{r}, t)\right\}_{t=t_{0}} & =\left\langle\Psi_{0}\left|\left[\hat{\mathbf{j}}(\mathbf{r}), \hat{H}\left(t_{0}\right)-\hat{H}^{\prime}\left(t_{0}\right)\right]\right| \Psi_{0}\right\rangle \\
= & \left\langle\Psi_{0}\left|\left[\hat{\mathbf{j}}(\mathbf{r}), \hat{v}_{\text {ext }}\left(\mathbf{r}, t_{0}\right)-\hat{v}_{\text {ext }}^{\prime}\left(\mathbf{r}, t_{0}\right)\right]\right| \Psi_{0}\right\rangle \\
& =\operatorname{in}\left(\mathbf{r}, t_{0}\right) \nabla\left\{v_{\text {ext }}\left(\mathbf{r}, t_{0}\right)-v_{\text {ext }}^{\prime}\left(\mathbf{r}, t_{0}\right)\right\}=n\left(\mathbf{r}, t_{0}\right) \nabla u_{0}(\mathbf{r}),
\end{aligned}
$$

which follows from the definition (2.6) together with the known evolution of the current density (2.7). If $u_{0}(\mathbf{r})$ is not a constant, then the right-hand side is non-zero, and consequently the derivatives of the current densities at $t_{0}$ must be distinct. The potential might also differ by a coefficient $u_{k}$ with $k \neq 0$; in this case one can take an appropriate higher time derivative

$$
\frac{d^{k+1}}{d t^{k+1}}\left\{\mathbf{j}(\mathbf{r}, t)-\mathbf{j}^{\prime}(\mathbf{r}, t)\right\}_{t=t_{0}}=n\left(\mathbf{r}, t_{0}\right) \nabla u_{k}(\mathbf{r}),
$$

with the non-constant $u_{k}(\mathbf{r})$ establishing hence that at least one term in the Taylor expansions of $\mathbf{j}(\mathbf{r}, t)$ and $\mathbf{j}^{\prime}(\mathbf{r}, t)$ differs. This implies that the current densities themselves deviate for $t>t_{0}$. In the second step one proves that the corresponding densities also differ. For this purpose one takes the $(k+1)$ st time derivative of the continuity equation (2.8) and again examine the difference

$$
\begin{aligned}
& \frac{\partial^{k+2}}{\partial t^{k+2}}\left\{n(\mathbf{r}, t)-n^{\prime}(\mathbf{r}, t)\right\}_{t=t_{0}}=-\nabla \cdot \frac{\partial^{k+1}}{\partial t^{k+1}}\left\{\mathbf{j}(\mathbf{r}, t)-\mathbf{j}^{\prime}(\mathbf{r}, t)\right\}_{t=t_{0}}= \\
&-\nabla \cdot\left\{n\left(\mathbf{r}, t_{0}\right) \nabla u_{k}(\mathbf{r})\right\}
\end{aligned}
$$

between the two systems. If the quantity on the right-hand side is non-zero, then the $(k+2)$ nd terms in the Taylor expansions of $n(\mathbf{r}, t)$ and $n^{\prime}(\mathbf{r}, t)$ around $t_{0}$ differ, and the densities themselves must hence deviate for $t>t_{0}$. The original proof [16] refers only to finite systems, where both the potential and the density decay to zero at large distances, but for extended systems it is easy to see that the right-hand side of (2.11) vanishes only if $u_{k}(\mathbf{r})$ is of the form

$$
u_{k}(\mathbf{r})=u_{k}(\mathbf{0})-\int_{\mathbf{0}}^{\mathbf{r}} \frac{n\left(\mathbf{0}, t_{0}\right)}{n\left(\mathbf{r}^{\prime}, t_{0}\right)} \mathbf{E}_{k} \cdot d \mathbf{r}^{\prime},
$$

with an arbitrary but constant vector $\mathbf{E}_{k}$. As the density is always positive, $u_{k}(\mathbf{r})$ then grows beyond all bounds as $|\mathbf{r}| \rightarrow \infty$, which implies that at least one of the potentials $v_{\text {ext }}(\mathbf{r}, t)$ or $v_{\text {ext }}^{\prime}(\mathbf{r}, t)$ becomes infinite. However, this case was explicitly excluded. For all finite physical potentials the right-hand side of (2.11) is indeed non-zero. This concludes the proof of the Runge-Gross theorem. Note that potentials of the type (2.12) are also incompatible with the Hohenberg-Kohn theorem in static density-functional theory: as the energy of the electrons can always be lowered by a translation in the direction of the field vector, there is no ground-state solution [39].

The Runge-Gross theorem is, in fact, a very strong statement: From the knowledge of the density alone it is possible to deduce the external potential and hence the manybody wave function, which in turn determines every observable of the system. Therefore, all observables can ultimately be regarded as functionals of the density. We note that, in contrast to more general cases $[40,41]$, there is no additional initial-state dependence in this scenario, because the stationary wave function at $t_{0}$ itself is determined by the static ground-state density $n_{0}(\mathbf{r})=n\left(\mathbf{r}, t_{0}\right)$ of the unperturbed system. 


\subsection{The time-dependent Kohn-Sham equations}

The Runge-Gross theorem states that all observables are functionals of the density, but it contains no prescription on how this central quantity can actually be calculated. To overcome the analogous problem in static density-functional theory, Kohn and Sham [3] suggested to use an auxiliary system of non-interacting electrons moving in an effective local potential, which is designed in such a way that the densities of the non-interacting system and the real interacting electrons coincide. This scheme has the big advantage to include the exact non-interacting kinetic energy, which represents almost all the true kinetic energy of the $N$-electron system. The main task is then to find a good approximation for this a priori unknown effective potential. This idea was generalized to the time-dependent case, where the Kohn-Sham electrons obey [16]

$$
\mathrm{i} \frac{\partial}{\partial t} \varphi_{j}(\mathbf{r}, t)=\left(-\frac{1}{2} \nabla^{2}+v_{\mathrm{KS}}[n](\mathbf{r}, t)\right) \varphi_{j}(\mathbf{r}, t)
$$

and the density is given by

$$
n(\mathbf{r}, t)=\sum_{j=1}^{N}\left|\varphi_{j}(\mathbf{r}, t)\right|^{2} .
$$

The Kohn-Sham scheme assumes that one can always find a local potential $v_{\mathrm{KS}}[n](\mathbf{r}, t)$ with the property that the orbitals obtained from (2.13) reproduce the given density of an interacting electron system, but the validity of this assumption, known as "non-interacting $v$-representability", is not obvious and requires a careful examination. If such a potential exists, however, then by virtue of the Runge-Gross theorem it is unique up to a purely timedependent function. Giving a constructive proof, van Leeuwen [42] showed that an effective local potential with the desired property exists if one can find a stationary wave function that yields the initial density $n\left(\mathbf{r}, t_{0}\right)$ and is the ground state of a non-interacting electron system. The problem is thus reduced to the question of non-interacting $v$-representability in static density-functional theory. Despite much progress, the latter is still unresolved. Examples of well-behaved densities that do not correspond to the ground state of a noninteracting system are known $[43,44]$; the implications of this discovery remain however unclear. In actual calculations, where the initial Kohn-Sham wave function is obtained from the constrained minimization of a smooth approximate energy functional, a solution can always be found [45].

If non-interacting $v$-representability is assumed, then $v_{\mathrm{KS}}[n](\mathbf{r}, t)$ is determined completely by the requirement that (2.14) equals the density of the real interacting electron system. As in the case of ground state DFT, one has then to find an explicit expression for the effective potential that can be exploited to construct useful approximations. For this purpose it is convenient to employ the same separation

$$
v_{\mathrm{KS}}[n](\mathbf{r}, t)=v_{\mathrm{ext}}(\mathbf{r}, t)+v_{\mathrm{H}}[n](\mathbf{r}, t)+v_{\mathrm{xc}}[n](\mathbf{r}, t)
$$

as in static density-functional theory. The first term is the external potential, the second is the Hartree potential

$$
v_{\mathrm{H}}[n](\mathbf{r}, t)=\int d^{3} r^{\prime} \frac{n\left(\mathbf{r}^{\prime}, t\right)}{\left|\mathbf{r}-\mathbf{r}^{\prime}\right|}
$$

and the third incorporates all remaining exchange and correlation effects. In the static case one can exploit the variational principle and determine the orbitals of the Kohn-Sham electrons in such a way that the total energy is minimized; all potential terms are then 
obtained as functional derivatives of the corresponding energy contributions with respect to the density. The energy in turn is a well defined physical quantity and amenable to approximations. In systems driven by time-dependent external fields the total energy is not a conserved quantity and there cannot be minimization principle. There exists, however, a quantity analogous to the energy, the quantum-mechanical action functional

$$
A[\Phi]=\int_{t_{0}}^{t_{1}} d t\left\langle\Phi(t)\left|\left(\mathrm{i} \frac{\partial}{\partial t}-\hat{H}(t)\right)\right| \Phi(t)\right\rangle,
$$

which has the property that its derivative with respect to a $N$-body function $\langle\Phi(t)|$ vanishes at the true many-body wave function, i.e., the solution of the Schrödinger equation

$$
\left.\frac{\delta A[\Phi]}{\delta\langle\Phi(t)|}\right|_{|\Phi(t)\rangle=|\Psi(t)\rangle}=\left(\mathrm{i} \frac{\partial}{\partial t}-\hat{H}(t)\right)|\Psi(t)\rangle=0 .
$$

Therefore, it is possible to solve the time-dependent problem by searching for the stationary point of the action. In contrast to the energy in the static case, the stationary point is not necessarily a minimum, however. Furthermore, the value of the action itself does not provide any relevant additional information, since for the true many-body wavefunction $A[\Psi]=0$.

By virtue of the Runge-Gross theorem we may consider the action as a functional of the density. The obvious definition of $A[n]$ is to evaluate $(2.17)$ at the wave function $\Phi[n](\{\mathbf{r}\}, t)$ that evolves from the given initial state and yields the density $n(\mathbf{r}, t)$. In analogy to the total energy in static density-functional theory, one would expect that the true density makes this functional stationary and can thus be identified. A suitable decomposition of the action would then define the exchange-correlation potential in terms of the functional derivative

$$
v_{\mathrm{xc}}(\mathbf{r}, t)=\frac{\delta A_{\mathrm{xc}}[n]}{\delta n(\mathbf{r}, t)} .
$$

Unfortunately, this procedure is doomed to failure. A first problem arises because the density determines the potential only up to a purely time-dependent function. Therefore, the wave function $\Phi[n](\{\mathbf{r}\}, t)$ and the value of the action derived from it are not unique. Even if the phase of the wave function is fixed by imposing additional constraints, there is another more fundamental problem, which becomes evident if one examines the second functional derivative

$$
\frac{\delta v_{\mathrm{xc}}(\mathbf{r}, t)}{\delta n\left(\mathbf{r}^{\prime}, t^{\prime}\right)}=\frac{\delta^{2} A_{\mathrm{xc}}[n]}{\delta n(\mathbf{r}, t) \delta n\left(\mathbf{r}^{\prime}, t^{\prime}\right)} .
$$

Whereas the expression on the right-hand side is symmetric in $(\mathbf{r}, t)$ and $\left(\mathbf{r}^{\prime}, t^{\prime}\right)$, the exchangecorrelation potential can only be influenced by the density at earlier times. Therefore, causality dictates that the left-hand side must vanish for $t<t^{\prime}$ but not for $t>t^{\prime}$. The symmetry and causality requirements contradict each other and cannot be satisfied simultaneously. One is hence forced to conclude that a differentiable functional $A_{\mathrm{xc}}[n]$ with the property (2.19) does not exist.

This causality dilemma [46, 47] was eventually resolved by van Leeuwen [45] using the time-contour method due to Keldysh [48]. In this approach to non-equilibrium dynamics the physical time $t$ is parametrized by an underlying parameter $\tau$ called pseudotime in such a way that $t(\tau)$ runs from $t_{0}$ to $t_{1}$ and back to $t_{0}$ if $\tau$ runs along the contour $C$ illustrated in figure 1. As pseudotime values on the forward and backward branches are distinct, the ordering along the contour differs from that on the physical time axis. The solution of the dilemma hence consists in satisfying the causality and symmetry requirements in different 


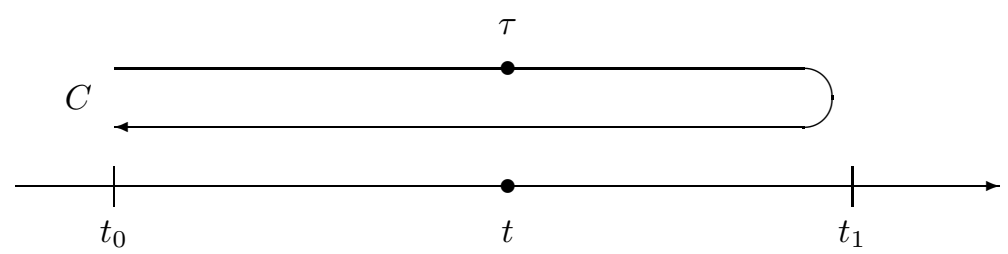

Figure 1. The Keldysh contour $C$, starting at $t_{0}$ and turning back at $t_{1}$. Pseudotime values $\tau$ on the forward and backward branches are distinct.

variable spaces. To this effect the action is first defined as a functional of the external potential in a form that does not explicitly contain $\partial / d t$

$$
\tilde{A}[U]=\mathrm{i} \ln \left\langle\Psi\left(t_{0}\right)\left|T_{C} \exp \left(-\mathrm{i} \int_{C} d \tau t^{\prime}(\tau) \hat{H}(\tau)\right)\right| \Psi\left(t_{0}\right)\right\rangle .
$$

For the derivation of (2.21) the reader can see Ref. [45]. The potential $U(\mathbf{r}, \tau)$ is contained in the Hamiltonian, and $T_{C}$ sorts the subsequent operators in order of ascending pseudotime arguments from right to left. For physical potentials of the form $U(\mathbf{r}, \tau)=v_{\text {ext }}(\mathbf{r}, t(\tau))$ the value of the action is zero, because the contributions along the two time-contour directions cancel each other, but its derivative can be non-zero. In fact, the action (2.21) is defined in such a way that its functional derivative yields the density

$$
\frac{\delta \tilde{A}[U]}{\delta U(\mathbf{r}, \tau)}=n(\mathbf{r}, \tau) \text {. }
$$

An unambiguous functional of the density can then be constructed by means of a Legendre transform

$$
A[n]=-\tilde{A}[U]+\int_{C} d \tau t^{\prime}(\tau) \int d^{3} r U(\mathbf{r}, \tau) n(\mathbf{r}, \tau) .
$$

Finally, for practical purposes the action is decomposed according to

$$
A[n]=A_{\mathrm{KS}}[n]-\frac{1}{2} \int_{C} d \tau t^{\prime}(\tau) \int d^{3} r \int d^{3} r^{\prime} \frac{n(\mathbf{r}, \tau) n\left(\mathbf{r}^{\prime}, \tau\right)}{\left|\mathbf{r}-\mathbf{r}^{\prime}\right|}-A_{\mathrm{xc}}[n] .
$$

The first term is the action of the non-interacting Kohn-Sham system, whose Legendre transform $\tilde{A}_{\mathrm{KS}}\left[U_{\mathrm{KS}}\right]$ is defined in analogy to (2.21) in terms of the initial Kohn-Sham wave function and the effective local potential. The second term is related to the Hartree potential, and the third gives rise to the exchange-correlation potential

$$
v_{\mathrm{xc}}(\mathbf{r}, t)=\left.\frac{\delta A_{\mathrm{xc}}[n]}{\delta n(\mathbf{r}, \tau)}\right|_{n=n(\mathbf{r}, t)} .
$$

Defining the action in the pseudotime domain instead of the real time axis guarantees the proper symmetry of the second functional derivative in $(\mathbf{r}, \tau)$ and $\left(\mathbf{r}^{\prime}, \tau^{\prime}\right)$. On the other hand, the exchange-correlation potential (2.25), which is obtained by inserting the physical time argument after performing the functional derivative with respect to $n(\mathbf{r}, \tau)$, respects causality on the time axis. From a theoretical point of view, all quantities that enter the Kohn-Sham scheme are thus well defined, and working approximations can be derived by finding a suitable expression for the action functional, for example through an expansion in powers of the Coulomb interaction. This approach is known as the time-dependent 
optimized effective-potential method [49]. Unfortunately, the leading term, which is linear in the Coulomb interaction and retains only exchange and no correlation [50], has already a high computational cost. In fact, at present the design of specific approximations for the exchange-correlation potential in TDDFT is still at a very early stage; we will discuss some promising approaches later in this review. Today, most calculations take however a pragmatic point of view and simply use one of the established functionals of static density-functional theory. The most popular choice is the adiabatic local-density approximation (ALDA) [15], which is obtained by evaluating the standard LDA potential with the time-dependent density $n(\mathbf{r}, t)$ :

$$
v_{\mathrm{xc}}^{\mathrm{ALDA}}[n](\mathbf{r}, t)=\left.v_{\mathrm{xc}}^{\mathrm{HEG}}[n](\mathbf{r}, t)\right|_{n=n(\mathbf{r}, t)} .
$$

The adiabatic approach is a drastic simplification, however, and a priori only justified for systems with a weak time-dependence that are always locally close to equilibrium. This adds to the problems that are due to the spatial locality of the LDA.

\subsection{Linear-response theory}

If the time-dependent external perturbation in $v_{\text {ext }}(\mathbf{r}, t)=v_{\text {ext }}^{(0)}(\mathbf{r})+v_{\text {ext }}^{(1)}(\mathbf{r}, t)$ is weak, then linear-response theory can be exploited to describe the dynamics of a system more efficiently than a full solution of the Kohn-Sham equations (2.13). In this case the density is expanded in orders of $v_{\text {ext }}^{(1)}(\mathbf{r}, t)$ according to $n(\mathbf{r}, t)=n^{(0)}(\mathbf{r})+n^{(1)}(\mathbf{r}, t)+\ldots$, where the first-order correction is given by

$$
n^{(1)}(\mathbf{r}, t)=\int_{-\infty}^{\infty} d t^{\prime} \int d^{3} r^{\prime} \chi\left(\mathbf{r}, \mathbf{r}^{\prime}, t-t^{\prime}\right) v_{\mathrm{ext}}^{(1)}\left(\mathbf{r}^{\prime}, t^{\prime}\right)
$$

in terms of the linear density-response function

$$
\chi\left(\mathbf{r}, \mathbf{r}^{\prime}, t-t^{\prime}\right)=\left.\frac{\delta n(\mathbf{r}, t)}{\delta v_{\mathrm{ext}}\left(\mathbf{r}^{\prime}, t^{\prime}\right)}\right|_{v_{\mathrm{ext}}\left(\mathbf{r}^{\prime}, t^{\prime}\right)=v_{\mathrm{ext}}^{(0)}\left(\mathbf{r}^{\prime}\right)} .
$$

Causality requires $\chi\left(\mathbf{r}, \mathbf{r}^{\prime}, t-t^{\prime}\right)=0$ for $t<t^{\prime}$, of course, because the density cannot be influenced by later variations of the potential. To calculate the linear density-response function in practice one exploits the fact that the density of the real system is identical to that of the non-interacting Kohn-Sham electrons. As the latter move in the effective potential $v_{\mathrm{KS}}\left(\mathbf{r}^{\prime \prime}, t^{\prime \prime}\right)$, one starts by applying the chain rule for functional derivatives

$$
\chi\left(\mathbf{r}, \mathbf{r}^{\prime}, t-t^{\prime}\right)=\int_{-\infty}^{\infty} d t^{\prime \prime} \int d^{3} r^{\prime \prime} \frac{\delta n(\mathbf{r}, t)}{\delta v_{\mathrm{KS}}\left(\mathbf{r}^{\prime \prime}, t^{\prime \prime}\right)} \frac{\delta v_{\mathrm{KS}}\left(\mathbf{r}^{\prime \prime}, t^{\prime \prime}\right)}{\delta v_{\mathrm{ext}}\left(\mathbf{r}^{\prime}, t^{\prime}\right)} .
$$

The first term on the right-hand side corresponds to the linear density-response function $\chi_{\mathrm{KS}}\left(\mathbf{r}, \mathbf{r}^{\prime \prime}, t-t^{\prime \prime}\right)$ of the non-interacting Kohn-Sham system, since the effective potential plays the role of the "external potential" of the Kohn-Sham system. It can be calculated explicitly from time-dependent perturbation theory and is given by

$$
\chi_{\mathrm{KS}}\left(\mathbf{r}, \mathbf{r}^{\prime \prime}, \omega\right)=\lim _{\eta \rightarrow+0} \sum_{j=1}^{\infty} \sum_{k=1}^{\infty}\left(f_{j}-f_{k}\right) \frac{\varphi_{j}^{*}(\mathbf{r}) \varphi_{k}(\mathbf{r}) \varphi_{j}\left(\mathbf{r}^{\prime \prime}\right) \varphi_{k}^{*}\left(\mathbf{r}^{\prime \prime}\right)}{\omega-\varepsilon_{k}+\varepsilon_{j}+\mathrm{i} \eta}
$$

in frequency space. The energies $\varepsilon_{j}$ appearing in the denominator are the eigenvalues of the unperturbed stationary Kohn-Sham wave functions $\varphi_{j}(\mathbf{r})$. In order to evaluate the second 
term in (2.29) one uses the separation (2.15), which yields

$$
\frac{\delta v_{\mathrm{KS}}\left(\mathbf{r}^{\prime \prime}, t^{\prime \prime}\right)}{\delta v_{\mathrm{ext}}\left(\mathbf{r}^{\prime}, t^{\prime}\right)}=\delta\left(\mathbf{r}^{\prime \prime}-\mathbf{r}^{\prime}\right) \delta\left(t^{\prime \prime}-t^{\prime}\right)+\frac{\delta v_{\mathrm{H}}\left(\mathbf{r}^{\prime \prime}, t^{\prime \prime}\right)}{\delta v_{\mathrm{ext}}\left(\mathbf{r}^{\prime}, t^{\prime}\right)}+\frac{\delta v_{\mathrm{xc}}\left(\mathbf{r}^{\prime \prime}, t^{\prime \prime}\right)}{\delta v_{\mathrm{ext}}\left(\mathbf{r}^{\prime}, t^{\prime}\right)}
$$

As both the Hartree potential and the exchange-correlation potential are functionals of the density, one can apply the chain rule once more and rewrite these two contributions as

$$
\frac{\delta v_{\mathrm{H}}\left(\mathbf{r}^{\prime \prime}, t^{\prime \prime}\right)}{\delta v_{\mathrm{ext}}\left(\mathbf{r}^{\prime}, t^{\prime}\right)}=\int_{-\infty}^{\infty} d t^{\prime \prime \prime} \int d^{3} r^{\prime \prime \prime} \frac{\delta v_{\mathrm{H}}\left(\mathbf{r}^{\prime \prime}, t^{\prime \prime}\right)}{\delta n\left(\mathbf{r}^{\prime \prime \prime}, t^{\prime \prime \prime}\right)} \frac{\delta n\left(\mathbf{r}^{\prime \prime \prime}, t^{\prime \prime \prime}\right)}{\delta v_{\mathrm{ext}}\left(\mathbf{r}^{\prime}, t^{\prime}\right)}
$$

and analogously for $\delta v_{\mathrm{xc}}\left(\mathbf{r}^{\prime \prime}, t^{\prime \prime}\right) / \delta v_{\text {ext }}\left(\mathbf{r}^{\prime}, t^{\prime}\right)$. The last term on the right-hand side of (2.32) is easily recognized as the linear density-response function $\chi\left(\mathbf{r}^{\prime \prime \prime}, \mathbf{r}^{\prime}, t^{\prime \prime \prime}-t^{\prime}\right)$. The functional derivative of the Hartree potential with respect to the density follows from the definition (2.16) and simply equals the Coulomb potential

$$
\frac{\delta v_{\mathrm{H}}\left(\mathbf{r}^{\prime \prime}, t^{\prime \prime}\right)}{\delta n\left(\mathbf{r}^{\prime \prime \prime}, t^{\prime \prime \prime}\right)}=\frac{1}{\left|\mathbf{r}^{\prime \prime}-\mathbf{r}^{\prime \prime \prime}\right|} \delta\left(t^{\prime \prime}-t^{\prime \prime \prime}\right)
$$

The last term of (2.31) contains the so-called exchange-correlation kernel

$$
f_{\mathrm{xc}}\left(\mathbf{r}^{\prime \prime}, \mathbf{r}^{\prime \prime \prime}, t^{\prime \prime}-t^{\prime \prime \prime}\right)=\left.\frac{\delta v_{\mathrm{xc}}\left(\mathbf{r}^{\prime \prime}, t^{\prime \prime}\right)}{\delta n\left(\mathbf{r}^{\prime \prime \prime}, t^{\prime \prime \prime}\right)}\right|_{n\left(\mathbf{r}^{\prime \prime \prime}, t^{\prime \prime \prime}\right)=n^{(0)}\left(\mathbf{r}^{\prime \prime \prime}\right)} .
$$

After collecting all terms and performing a Fourier transform to frequency space, whereby convolutions on the time axis turn into simple multiplications, one obtains the final integral equation $[20]$

$$
\begin{aligned}
& \chi\left(\mathbf{r}, \mathbf{r}^{\prime}, \omega\right)=\chi_{\mathrm{KS}}\left(\mathbf{r}, \mathbf{r}^{\prime}, \omega\right) \\
&+\int d^{3} r^{\prime \prime} \int d^{3} r^{\prime \prime \prime} \chi_{\mathrm{KS}}\left(\mathbf{r}, \mathbf{r}^{\prime \prime}, \omega\right)\left(\frac{1}{\left|\mathbf{r}^{\prime \prime}-\mathbf{r}^{\prime \prime \prime}\right|}+f_{\mathrm{xc}}\left(\mathbf{r}^{\prime \prime}, \mathbf{r}^{\prime \prime \prime}, \omega\right)\right) \chi\left(\mathbf{r}^{\prime \prime \prime}, \mathbf{r}^{\prime}, \omega\right) .
\end{aligned}
$$

The TDDFT equations in the linear-response regime can be cast in numerous different forms. For solids, in most implementations the integral equation (2.35) is solved routinely by projecting all quantities onto a suitable set of basis functions. Very often, one uses a plane wave representation within the pseudotential approximation (see e.g. [51, 52]), but localized basis sets can equally be used to allow for all-electron calculations (see e.g. [53]). Equation (2.35) thus turns into a matrix equation $\chi(\omega)=\chi_{\mathrm{KS}}(\omega)+\chi_{\mathrm{KS}}(\omega)\left[v+f_{\mathrm{xc}}(\omega)\right] \chi(\omega)$ for example in reciprocal lattice vectors in case of periodic systems where $\chi=\chi_{\mathbf{G}, \mathbf{G}^{\prime}}(\mathbf{q})$. If one wishes only obtain an absorption spectrum or the loss function at a given momentum transfer (see definitions in section 3) only one component of the matrix $\chi_{\mathbf{G G}^{\prime}}(\mathbf{q}, \omega)$ is required. This can be obtained by solving a linear system, thus avoiding a numerically involved matrix inversion [51].

Alternatively, absorption spectra can be calculated by propagating the full TD KohnSham equations in real-time [54]. This description decreases storage requirements, it allows the entire frequency-dependent dielectric function to be calculated at once, and the scaling with the number of atoms is quite favorable. However, the prefactor is fairly large as such calculations typically require $\approx 10000$ time-steps with a time-step of $\approx 10^{-3}$ fs [55].

Another efficient approach, based on linear-response within Ghosh and Dhara's timedependent density functional formalism [56], was proposed [57]. It uses an iterative scheme 
in real-space, in which the density and the potential are updated in each cycle, thereby avoiding the explicit evaluation of the Kohn-Sham response kernels.

Finally, a method to calculate the dynamical polarizability using only occupied states has been proposed recently [58]. The dynamical polarizability is represented by a matrix continued fraction whose coefficients can be obtained from a Lanczos method. This methods scales favorably with system size, and it may become useful for large scale systems. At present there is however only a single application to the benzene molecule [58].

\subsection{Excitation energies}

In static DFT the interpretation of the one-particle Kohn-Sham eigenvalues $\varepsilon_{j}$ as quasiparticle energies is not formally justified and it leads to the well known problem of the underestimation of transition energies. In the framework of TDDFT the relevant information about the excited states is contained in the linear density-response function: in fact it can be shown that the true excitation energies are the poles of $\chi\left(\mathbf{r}, \mathbf{r}^{\prime}, \omega\right)$. In contrast to other attempts to calculate electronic excitations within a density-functional framework [59, 60, 61], TDDFT has the great advantage that it is not restricted to a subset of excited states but, in principle, yields the complete excitation spectrum.

In order to see this, one can calculate the density charge due to the external potential at first order. The stationary eigenstates of the original unperturbed Hamiltonian are labeled by $\Psi_{j}(\{\mathbf{r}\}, t)=\Psi_{j}(\{\mathbf{r}\}) \exp \left(-\mathrm{i} E_{j} t\right)$, where $E_{j}$ denotes the corresponding energy eigenvalues. After the onset of the time-dependent perturbation it is possible to expand the wave function $\Psi(\{\mathbf{r}\}, t)$ that evolves from the ground state $\Psi^{(0)}(\{\mathbf{r}\}, t)=\Psi_{0}(\{\mathbf{r}\}) \exp \left(-\mathrm{i} E_{0} t\right)$ in orders of $v_{\text {ext }}^{(1)}(\mathbf{r}, t)$. The first-order correction is

$$
\begin{aligned}
& \Psi^{(1)}(\{\mathbf{r}\}, t)=-\mathrm{i} \sum_{j=0}^{\infty} \Psi_{j}(\{\mathbf{r}\}, t) \int_{-\infty}^{t} d t^{\prime} \int d^{3} r_{1}^{\prime} \ldots \int d^{3} r_{N}^{\prime} \Psi_{j}^{*}\left(\left\{\mathbf{r}^{\prime}\right\}, t^{\prime}\right) \\
&\left(\sum_{i=1}^{N} v_{\text {ext }}^{(1)}\left(\mathbf{r}^{\prime}{ }_{i}, t^{\prime}\right)\right) \Psi_{0}\left(\left\{\mathbf{r}^{\prime}\right\}, t^{\prime}\right) .
\end{aligned}
$$

The corresponding change in the density is

$$
\begin{aligned}
n^{(1)}(\mathbf{r}, t)=N \int d^{3} r_{2} \ldots \int d^{3} r_{N}\{ & {\left[\Psi^{(1)}\left(\mathbf{r}, \mathbf{r}_{2}, \ldots, \mathbf{r}_{N}, t\right)\right]^{*} \Psi^{(0)}\left(\mathbf{r}, \mathbf{r}_{2}, \ldots, \mathbf{r}_{N}, t\right)+} \\
& {\left.\left[\Psi^{(0)}\left(\mathbf{r}, \mathbf{r}_{2}, \ldots, \mathbf{r}_{N}, t\right)\right]^{*} \Psi^{(1)}\left(\mathbf{r}, \mathbf{r}_{2}, \ldots, \mathbf{r}_{N}, t\right)\right\} . }
\end{aligned}
$$

In order to simplify the notation we introduce the overlap functions

$$
n_{j}(\mathbf{r})=N \int d^{3} r_{2} \ldots \int d^{3} r_{N} \Psi_{0}^{*}\left(\mathbf{r}, \mathbf{r}_{2}, \ldots, \mathbf{r}_{N}\right) \Psi_{j}\left(\mathbf{r}, \mathbf{r}_{2}, \ldots, \mathbf{r}_{N}\right),
$$

and after inserting (2.36) and (2.38) into (2.37) we obtain

$$
\begin{aligned}
n^{(1)}(\mathbf{r}, t)=\int_{-\infty}^{\infty} d t^{\prime} \int d^{3} r^{\prime} & {\left[-\mathrm{i} \sum_{j=0}^{\infty}\left(n_{j}(\mathbf{r}) n_{j}^{*}\left(\mathbf{r}^{\prime}\right) e^{-\mathrm{i}\left(E_{j}-E_{0}\right)\left(t-t^{\prime}\right)}\right.\right.} \\
& \left.\left.-n_{j}^{*}(\mathbf{r}) n_{j}\left(\mathbf{r}^{\prime}\right) e^{\mathrm{i}\left(E_{j}-E_{0}\right)\left(t-t^{\prime}\right)}\right) \Theta\left(t-t^{\prime}\right)\right] v_{\text {ext }}^{(1)}\left(\mathbf{r}^{\prime}, t^{\prime}\right) .
\end{aligned}
$$


Comparing this expression with (2.27), one finds that the term in square brackets equals the linear density-response function $\chi\left(\mathbf{r}, \mathbf{r}^{\prime}, t-t^{\prime}\right)$. The Heaveside step function $\Theta\left(t-t^{\prime}\right)$ has been introduced to replace the integral over time $\int_{-\infty}^{t} d t^{\prime}$ with $\int_{-\infty}^{\infty} d t^{\prime}$. After a Fourier transform to frequency space, and using $\Theta(t)=\mathrm{i} / 2 \pi \lim _{\eta \rightarrow 0+} \int_{-\infty}^{\infty} d \omega \frac{1}{\omega+\mathrm{i} \eta} e^{-\mathrm{i} t \omega}$ one arrives at the Lehmann representation of the density response function:

$$
\chi\left(\mathbf{r}, \mathbf{r}^{\prime}, \omega\right)=\lim _{\eta \rightarrow 0+} \sum_{j=1}^{\infty}\left(\frac{n_{j}(\mathbf{r}) n_{j}^{*}\left(\mathbf{r}^{\prime}\right)}{\omega-E_{j}+E_{0}+\mathrm{i} \eta}-\frac{n_{j}^{*}(\mathbf{r}) n_{j}\left(\mathbf{r}^{\prime}\right)}{\omega+E_{j}-E_{0}+\mathrm{i} \eta}\right)
$$

where $\eta$ is a positive infinitesimal. From (2.40) it is evident that the poles of $\chi\left(\mathbf{r}, \mathbf{r}^{\prime}, \omega\right)$ correspond to the exact excitation energies $E_{j}-E_{0}$. Furthermore, all quantities on the righthand side depend only on the Hamiltonian of the unperturbed stationary system. By virtue of the Hohenberg-Kohn theorem the linear density-response function is hence a functional of the static ground-state density $n_{0}(\mathbf{r})$.

The form of (2.40) is valid for finite systems with discrete eigenvalues. As the energies $E_{j}$ of the eigenstates of the many-electron system are real, it appears that the poles of $\chi\left(\mathbf{r}, \mathbf{r}^{\prime}, \omega\right)$ are at real energies. For extended systems, on the other hand, the spectrum is continuous, and the sum in (2.40) turns into an integral that gives rise to a branch cut along the real energy axis. The infinitely close-lying resonances thus merge into broad structures that can be identified with elementary quasiparticles, such as plasmons or excitons. As these structures have a certain width, they are described by poles in the complex plane with a real part, which corresponds to the energy of the excitation, and a finite imaginary part, whose inverse is proportional to the excitation lifetime.

\section{TDDFT in practise: approximations and problems}

Linear response theory can be applied now to study the response of an extended system to a small time-dependent perturbation $v_{\text {ext }}(\mathbf{r}, t)$. The linear variation of the density induced by the perturbation is given by (2.27). As a consequence of the polarisation of the system due to the applied perturbation, the total potential becomes a sum of the external potential and the induced potential: $v_{\text {tot }}=v_{\text {ext }}+v_{\text {ind }}$. The basic quantity that gives information about the screening of the system in linear response is the microscopic dielectric function $\epsilon$, that relates the total potential $v_{\text {tot }}$ to the applied potential $v_{\text {ext }}$ :

$$
v_{\text {tot }}(\mathbf{r}, t)=\int_{-\infty}^{\infty} d t^{\prime} \int d^{3} r^{\prime} \epsilon^{-1}\left(\mathbf{r}, \mathbf{r}^{\prime}, t-t^{\prime}\right) v_{\mathrm{ext}}\left(\mathbf{r}^{\prime}, t^{\prime}\right)
$$

The microscopic dielectric function $\epsilon$ and the reducible polarizability $\chi$ are hence related by

$$
\epsilon^{-1}\left(\mathbf{r}, \mathbf{r}^{\prime}, t-t^{\prime}\right)=\delta\left(\mathbf{r}-\mathbf{r}^{\prime}\right) \delta\left(t-t^{\prime}\right)+\int d^{3} r^{\prime \prime} v\left(\mathbf{r}-\mathbf{r}^{\prime \prime}\right) \chi\left(\mathbf{r}^{\prime \prime}, \mathbf{r}^{\prime}, t-t^{\prime}\right)
$$

For periodic systems, the most natural way to deal with spatial periodicity is to apply a Fourier transform and rewrite (3.2) in reciprocal space

$$
\epsilon_{\mathbf{G G}^{\prime}}^{-1}(\mathbf{q}, \omega)=\delta_{\mathbf{G G}^{\prime}}+v_{\mathbf{G}}(\mathbf{q}) \chi_{\mathbf{G G}^{\prime}}(\boldsymbol{q}, \omega),
$$

where $\mathbf{G}$ is a vector of the reciprocal lattice, while $\mathbf{q}$ is a vector in the first Brillouin zone. In (3.3) a Fourier transform has also been applied to move from time to frequency space. 
From the microscopic dielectric function one has to obtain measurable quantities. In the case of absorption spectra, this means to calculate the imaginary part of the macroscopic dielectric function $[62,63,64]$

$$
\epsilon_{\mathrm{M}}(\omega)=\lim _{\mathbf{q} \rightarrow 0} \frac{1}{\left[\epsilon_{\mathbf{G G}^{\prime}}^{-1}(\mathbf{q}, \omega)\right]_{\mathbf{G}, \mathbf{G}^{\prime}=0}} .
$$

We have dealt so far with the response to a potential whose electric field is longitudinal with respect to the wavevector. Light, instead, is a transverse perturbation, i.e. its electric field is perpendicular to the wavevector. Hence, it would seem inappropriate to use the present treatment. However, since the light wavevector is very small, one can think to rotate it such as to have it parallel to the electric field and apply the present formalism [65]. The validity of this approach has been rigorously demonstrated for cubic crystals in Ref. [13]. Furthermore, we observe that, in general, for anisotropic systems (3.3) and (3.4) depend on the direction of the vector $\mathbf{q}$ (i.e. on the polarisation of the incoming radiation), thus both microscopic and macroscopic responses are described by a dielectric tensor, instead of simple scalar functions.

The same quantity $\epsilon_{\mathrm{M}}$ is also related to electron energy loss spectra (EELS) for vanishing momentum transfer, through the loss function $-\operatorname{Im}\left\{1 / \epsilon_{\mathrm{M}}\right\}$. For non-vanishing momentum transfer $\mathbf{Q}=\mathbf{q}+\mathbf{G}$, the loss function is $-\operatorname{Im}\left\{\epsilon_{\mathbf{G G}}^{-1}(\mathbf{q}, \omega)\right\}$. In this case the longitudinal formulation of the dielectric response is obviously appropriate. From (3.3) it follows that the loss function can be related to the linear density response function $\chi$ :

$$
\operatorname{EEL}(\mathbf{q}+\mathbf{G}, \omega)=-v_{\mathbf{G}}(\mathbf{q}) \operatorname{Im}\left\{\chi_{\mathbf{G G}}(\mathbf{q}, \omega)\right\},
$$

where the $\mathbf{G G}^{\prime}$-matrix $\chi$ can be obtained by solving the Dyson-like screening equation (2.35). In (2.35) the full response function is expressed in terms of the independent-particle $\chi_{\mathrm{KS}}$ via a kernel composed by two terms, the bare Coulomb potential and the exchangecorrelation contribution $f_{\mathrm{xc}}$. A similar expression can be written also in the case of the optical absorption, provided one builds a modified response function $\bar{\chi}$ :

$$
\operatorname{Im}\left\{\epsilon_{\mathrm{M}}\right\}=-\lim _{\mathbf{q} \rightarrow 0} v_{\mathbf{G}}(\mathbf{q}) \operatorname{Im}\left\{\bar{\chi}_{\mathbf{G G}^{\prime}}\right\}
$$

which satisfies the Dyson-like screening equation

$$
\bar{\chi}=\chi_{\mathrm{KS}}+\chi_{\mathrm{KS}}\left(\bar{v}+f_{\mathrm{xc}}\right) \bar{\chi},
$$

where the modified Coulomb interaction is defined as

$$
\bar{v}_{\mathbf{G}}=\left\{\begin{array}{ll}
v_{\mathbf{G}} & \text { for } \quad \mathbf{G} \neq 0 \\
0 & \text { for } \quad \mathbf{G}=0
\end{array} .\right.
$$

Following [14], the description of both absorption and EELS for $\mathbf{q} \rightarrow 0$ can be unified by introducing the generalised spectrum $A(\omega)$ and a generalised function $X_{\mathbf{G G}^{\prime}}(\mathbf{q}, \omega)$. The function $X$ stands for the modified response function $\bar{\chi}$ in the case of absorption and for the reducible response function $\chi$ in the case of EEL:

$$
\left.\begin{array}{c}
\text { Abs } \\
\operatorname{EEL}
\end{array}\right\}=A(\omega)=-\operatorname{Im}\left\{\lim _{\mathbf{q} \rightarrow 0} v_{\mathbf{G}=0}(\mathbf{q}) X_{\mathbf{G}=\mathbf{G}^{\prime}=0}(\mathbf{q}, \omega)\right\} .
$$

In any formulation, the basic ingredients to obtain either the absorption or the EELS are the Kohn-Sham eigenfunctions and eigenenergies that enter the expression for the independentparticle Kohn-Sham response function $\chi_{\mathrm{KS}}(2.30)$. These are usually obtained through a 
ground-state DFT calculation using an approximate exchange-correlation potential. In the total kernel of (2.35) and (3.7) $\bar{v}$ accounts for classical depolarisation effects (also known as crystal local field effects (LFE) in a solid). It reflects the microscopic induced Hartree potential created by polarizable inhomogeneities in the system. The apparently subtle difference between absorption and EEL, i.e. the inclusion or the exclusion of the long-range term $v_{\mathbf{0}}$, is crucial for extended systems - for example, $v_{\mathbf{0}}$ is responsible for the plasmons - , whereas its contribution in finite systems becomes vanishingly small [66]. The term $f_{\mathrm{xc}}$ is a complex quantity that contains all non-trivial many-body effects. Its analytical expression is unknown.

We have therefore two key approximations: (i) the ground-state exchange-correlation potential, and (ii) the exchange-correlation kernel. Of course, these two quantities are in principle linked, due to the fact that the exchange-correlation kernel is the functional derivative of the time dependent exchange-correlation potential. The relative importance of the two approximations depends, as we will see in the following, on the physical system under study. For example, when dealing with finite systems it is often essential to have good Kohn-Sham eigenstates - and therefore a good ground-state exchange-correlation potential - while the role of the exchange-correlation kernel is less relevant [67, 22]. The opposite is usually true for extended systems, where a good approximation to the exchange-correlation kernel turns out to be essential, especially when it comes to describe optical absorption spectra [14].

Searching for approximations several approaches are possible. For the ground-state exchange-correlation potential, 40 years of development have led to a swarm of functionals (for more information, see one of the numerous review articles on the subject, e.g. [68]). For the exchange-correlation kernel, one can either look for a good approximation for the time-dependent exchange-correlation potential and then use the definition of $f_{\mathrm{xc}}(2.34)$ or find directly an expression for the exchange-correlation kernel. Either choice has clear advantages and disadvantages. On the one hand, the exchange-correlation kernel is a simpler object, in the sense that it is a functional of the ground-state density, and is therefore amenable to more controllable approximations. On the other hand, if we are in possession of a good time-dependent exchange-correlation functional, we can tackle both linear and non-linear response properties. Instead, only linear response is accessible through the knowledge of the exchange-correlation kernel. Most often, the standard approximations used for $v_{\mathrm{xc}}(\mathbf{r}, t)$ are adiabatic, and lead to very simple $f_{\mathrm{xc}}$ 's.

\subsection{Basic approximations: $R P A$ and $T D L D A$}

The lowest level approximation to perform real calculations consists in setting to zero the terms in (2.35) and (3.7) coming from the microscopic components of the induced Hartree potential $(\bar{v}=0)$ and the variations of the exchange-correlation potential $\left(f_{\mathrm{xc}}=0\right)$. By comparing (3.4) with (3.6), with vanishing $\bar{v}$ and $f_{\mathrm{xc}}$, it is possible to see that this is equivalent to neglect all the off-diagonal components of the matrix $\epsilon_{\mathbf{G G}^{\prime}}^{-1}$. We will refer to this as the independent particle approximation (IPA). The excitation energies in the IPA are simply given by the differences between the eigenenergies of the unoccupied and occupied KohnSham states, which are used to build $\chi_{\mathrm{KS}}$. Independently of the quality of the states entering in $\chi_{\mathrm{KS}}$, this usually leads to absorption peaks that are systematically red-shifted in relation to the experimental spectra [14]. This is a consequence of the well known gap problem of DFT: the gap between filled and empty states is substantially underestimated [69].

By neglecting only the exchange-correlation kernel we obtain the so-called random-phase 
approximation (RPA) [63, 64]

$$
f_{\mathrm{xc}}^{\mathrm{RPA}}=0 .
$$

In this case, the only part of the total kernel in (2.35) and (3.7) which is taken into account is the classical Coulomb term. This term describes the well known Lindhard theory of screening with the addition of LFE [70]. Although very simple, the RPA yields results of reasonable accuracy for a wide-range of systems, and it is still widely employed in actual calculations. We will see some examples in the following of this section.

In the next step in the ladder of complexity come the already mentioned adiabatic approximations. At the level of the time-dependent exchange-correlation potential the adiabatic approximation implies

$$
v_{\mathrm{xc}}^{\text {adiabatic }}[n](\mathbf{r}, t)=\tilde{v}_{\mathrm{xc}}[n(t)](\mathbf{r}, t),
$$

where $\tilde{v}_{\mathrm{xc}}[n]$ is some given ground-state exchange-correlation functional. Note that, regardless of the choice of $\tilde{v}_{\mathrm{xc}}[n]$, the resulting kernel is instantaneous: $f_{\mathrm{xc}}\left(\mathbf{r}, \mathbf{r}^{\prime}, t, t^{\prime}\right)=$ $\delta\left(t-t^{\prime}\right) f_{\mathrm{xc}}\left(\mathbf{r}, \mathbf{r}^{\prime}\right)$, i.e., its Fourier transform is frequency independent (it can be of course non-local in space). If the LDA static potential is inserted in (3.11), one obtains the most common functional of TDDFT: the ALDA potential. Using definition (2.34), one can then derive the local and static ALDA (also called TDLDA) exchange-correlation kernel [17, 18]

$$
f_{\mathrm{xc}}^{\mathrm{TDLDA}}\left(\mathbf{r}, \mathbf{r}^{\prime}, t, t^{\prime}\right)=\left.\delta\left(\mathbf{r}-\mathbf{r}^{\prime}\right) \delta\left(t-t^{\prime}\right) \frac{d^{2} e_{\mathrm{xc}}^{H E G}(n)}{d n^{2}}\right|_{n=n_{\mathrm{GS}}(\mathbf{r})},
$$

where $e^{\mathrm{HEG}}$ is the energy per unit volume of the homogeneous electron gas, and $n_{\mathrm{GS}}$ is the ground-state electron density.

It is clear that the TDLDA retains all the problems already present in the LDA. The most important of these are perhaps, for neutral finite systems, the incorrect asymptotic behaviour of the LDA potential (instead of decaying as $-1 / r$, the LDA exchange-correlation potential goes to zero exponentially) and, for infinite systems, its local dependence on the density. These drawbacks can not be corrected by using in (3.11) most of the GGAs [71], nor the more modern meta-GGAs functionals [72, 73].

Nevertheless, for the calculation of optical response spectra in large variety of finite systems, the TDLDA has proved to be able to reproduce low energy peaks with an accuracy of around $0.1-0.4 \mathrm{eV}$ [74]. For solids, the situation is a bit more complicated. EEL or X-ray scattering (IXS) spectra are often of good quality, especially when the transferred momentum $\mathbf{q}$ is finite $[75,26,25]$. Instead, the description of $\operatorname{Im}\left\{\epsilon_{M}(\omega)\right\}$ for vanishing momentum transfer, which is the case in optical response, is perhaps the best known failure of TDLDA. Note that in an extended system the TDLDA kernel for a vanishing $\mathbf{q}$ yields always a relatively small correction to the RPA results, because it is constant for $q \rightarrow 0$ and multiplied with $\chi_{\mathrm{KS}}$ that goes to 0 as $q^{2}$. It can hence only have an effect via the LFE [27]. We will discuss this issue more in detail in the following of this section.

It is clear from the above that the behaviour of the different approximations depends strongly on the spectroscopy and on the dimensionality of the physical system. Therefore, it is interesting to present an overview of results for finite systems (molecules, clusters) to be compared to analogous results for infinite systems. In view of that, the following two subsections handle separately optical absorption in finite (section 3.2) and periodic systems (section 3.3). We then turn to EEL and IXS in section 3.4. Finally, we try to understand the failures of the TDLDA (section 3.5), and discuss possible routes to overcome them. 


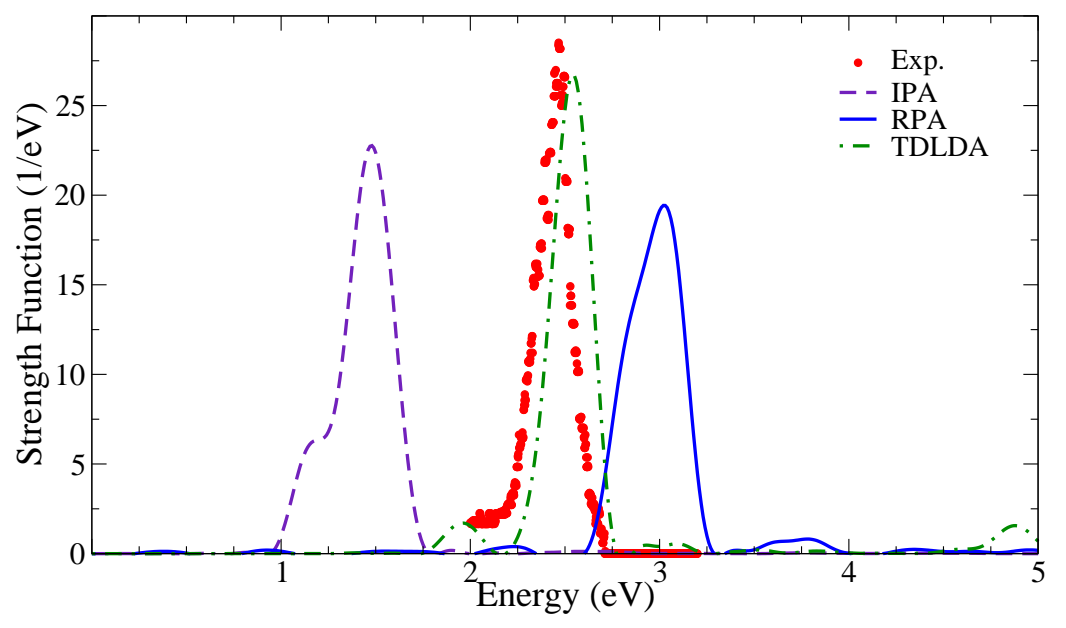

Figure 2. Photoabsorption spectrum for a Na8 cluster [14]. Dots: experimental data; dashed line:IPA Kohn-Sham transitions; solid line: RPA; dashed-dotted line: TDLDA.

\subsection{Finite systems}

The first calculations of excitation energies within TDDFT were performed before the formal demonstration of the Runge-Gross theorem. In 1977 Ando determined intersubband transitions in semiconductor heterostructures [76]. Shortly after, Zangwill and Soven [15] applied TDLDA to the calculation of the photo absorption cross section of rare gas atoms, obtaining a very good agreement with experimental data.

However, it was only in recent years that TDDFT became one of the most popular tools for the calculation of excitation properties. By now, the TDLDA kernel (3.12) has been successfully applied to atoms, organic and biological molecules, metallic and semiconducting clusters, fullerenes, etc $[77,78,79,74,80]$. Besides some more problematic cases (see section 3.5) the calculated excitation energies and absorption spectra are, in general, in excellent agreement with available experimental data. From the plethora of available applications of TDDFT to finite systems [81], we show here only two illustrative examples: (i) $\mathrm{Na}_{8}$, a prototype metallic cluster, and (ii) the polycyclic aromatic hydrocarbons (PAHs).

The absorption spectra of a $\mathrm{Na}_{8}$ cluster [14] are shown in figure 2. The IPA calculation (dashed line) is compared to a RPA (solid line) and a TDLDA result (dashed-dotted line). The experimental spectrum [82] is also plotted (dots). As a general feature, present in both metallic and semiconducting clusters, we find that the RPA peaks are blue-shifted with respect to the IPA peaks. Adding the TDLDA kernel brings a further correction, this time as a shift towards lower energies. The resulting transition energies then accurately reproduce the experiment. It is important to observe that, already in the RPA, absorption at low energies is correctly suppressed with respect to IP calculations. In fact this suppression of the oscillator strength is essentially due to the induced classical depolarisation potential. From (3.4) it can be observed that the LFE come from the off-diagonal terms of the matrix $\epsilon_{\mathbf{G G}^{\prime}}$. In other words, they express the fact that the electronic response of an inhomogeneous structure is position-dependent (and not only distance-dependent). It is intuitive that such an effect has to be the stronger the larger the inhomogeneity of the system. Since a finite object represents a strong inhomogeneity in the otherwise empty space, it is not surprising that the LFE are particularly important for this kind of systems. 


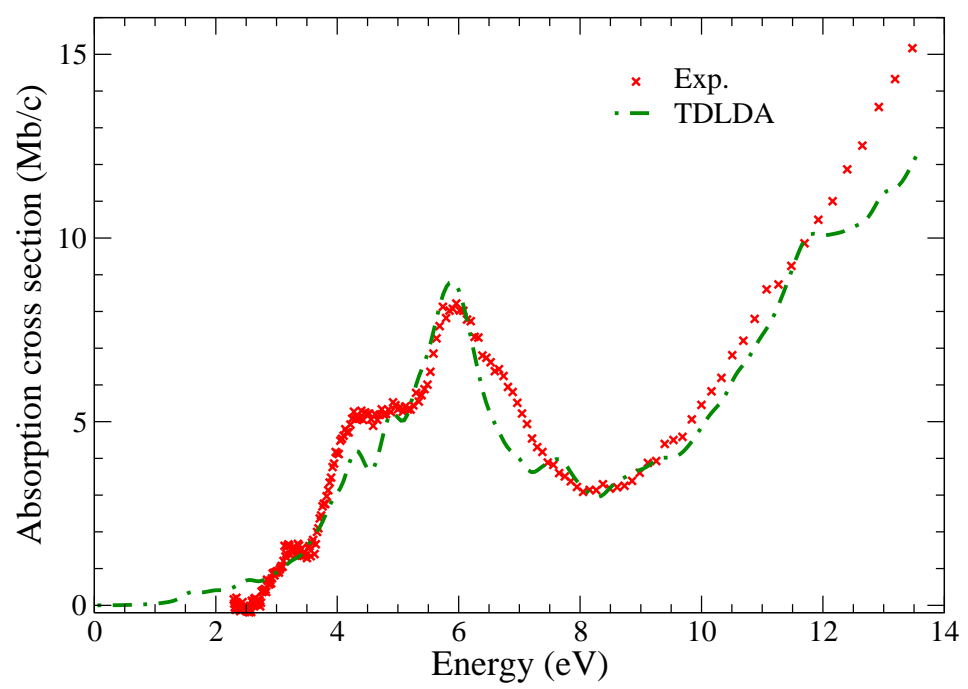

Figure 3. Comparison between the calculated weighted sum of the spectra[83] (dotteddashed line) for neutral PAHs and the experimental results of Joblin et al [84](crosses) for a natural mixture of neutral PAHs with average $N_{\mathrm{C}}=24$.

To illustrate the wide range of applicability of TDDFT, we now turn to the modelling of interstellar photophysics [83]. Due to their spectral properties, their high photostability and the fact that they are carbon-based, free gas-phase PAHs (in different charge and hydrogenation states) are commonly thought to be an important component of the interstellar medium. However, to compare with the measured spectrum of the interstellar medium, laboratory data of the individual PAHs are necessary. These are, however, often scarce and difficult to obtain. An alternative are numerical experiments based on TDDFT.

In figure 3 the dotted-dashed line represents the calculation by Malloci et al [83] of the photo-absorption cross-section up to the vacuum ultraviolet for a mixture of 20 different neutral PAHs, ranging in size from naphthalene $\left(\mathrm{C}_{10} \mathrm{H}_{8}\right)$ to dicoronylene $\left(\mathrm{C}_{48} \mathrm{H}_{20}\right)$. The overall spectrum for the PAH mixture is obtained as a weighted sum of the spectra for the single $\mathrm{C}_{n} \mathrm{H}_{m}$ molecules. (The statistical weights are assumed to be inversely proportional to the total number of carbon atoms $N_{\mathrm{C}}$ of each molecule. The average $N_{\mathrm{C}}$ of the theoretical sample is $N_{\mathrm{C}}=23.55$ ). The experimental spectrum measured by Joblin et al [84] for a natural mixture of neutral PAHs with average $N_{\mathrm{C}}=24$ is also plotted in figure 3 (crosses). Two distinct features can be observed in the spectrum: (i) a collective broad absorption peak, resulting from the sum of the $\pi \rightarrow \pi^{*}$ transitions at $\simeq 6 \mathrm{eV}$, and characterised by distinct structures due to the coincidence of relatively strong transitions in different molecules; and (ii) a smooth far-UV rise. The agreement between theory and experiment is very good and validates the use of TDLDA calculations as a substitute for laboratory data when the latter are lacking.

We have just witnessed snapshots of the quality of TDLDA calculations for finite systems. However, it should not be forgotten that in some cases TDLDA is not adequate to describe excitations of finite systems: a typical example is the failure in reproducing Rydberg series [22]. Moreover, when the molecules becomes more extended, this quality in general degrades. An example is the calculation of optical properties of long conjugated polymers $[24,85]$. The problem is related to a non-local dependence of the exchange-correlation 


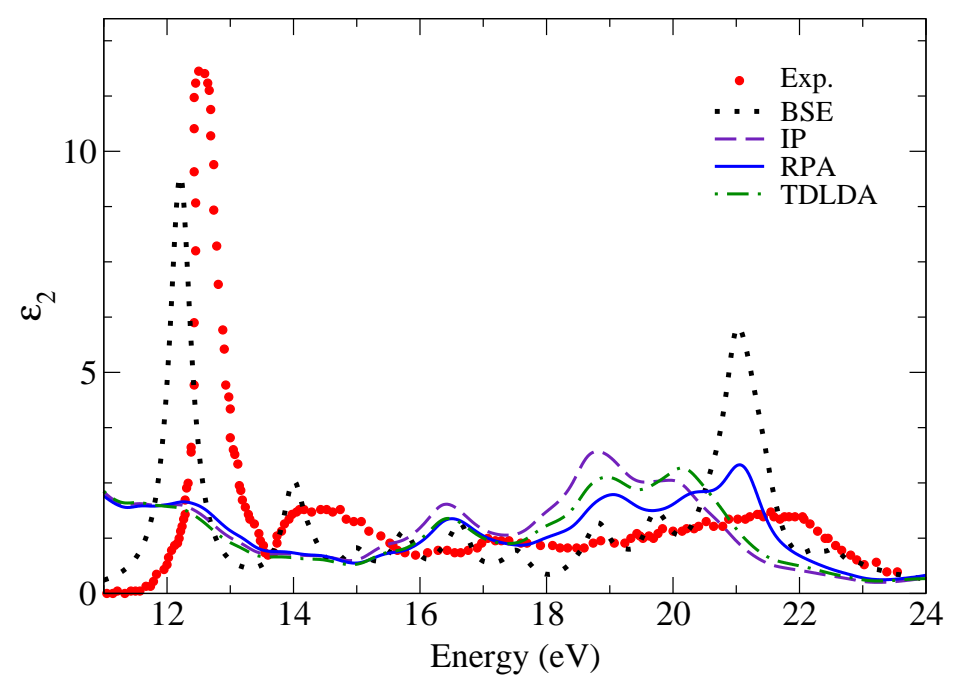

Figure 4. Imaginary part of the macroscopic dielectric function for LiF [87]. Dots: experiment [88]; dotted-line: BSE calculation; dashed line: IPA calculation; solid line: RPA calculation; dot-dashed line: TDLDA calculation.

potential: In a system with an applied electric field, the exact exchange-correlation potential develops a linear part that counteracts the applied field [24, 86]. This term is completely absent in both the LDA and the GGA. It is present in more non-local functionals like the EXX (see section 7).

In the following we will investigate more closely this problem.

\subsection{Optical absorption in extended systems}

The simplest approach to the optical properties of semiconductors or wide-gap insulators within TDDFT is the TDLDA. In view of the excellent quality of the results obtained for the photoabsorption of clusters, one could perhaps expect that the same would occur for extended systems. This is unfortunately not the case. As we can see in figure 4 for the optical absorption of a LiF crystal, the TDLDA (dash-dotted line) induces only some minor modifications with respect to the RPA (solid line), and both are very far from the experimental curve (dots). The largest disagreement concerns the absence of the strong excitonic peak at about $12.5 \mathrm{eV}$. Good agreement can be found using the many-body BetheSalpeter approach (dotted line) at the price of a significantly larger computational effort [89, 90, 91, 92]: in that framework, electron addition and removal energies as well as the electron-hole interaction are explicitly calculated within many-body Green's function theory (see section 7).

This situation is quite general and is found in a wide range of semiconductors $(\mathrm{Si}-\mathrm{see}$ also the inset of figure 5 - Ge, GaAs, etc.) and wide-band gap semiconductors or insulators (diamond, $\mathrm{MgO}, \mathrm{SiO}_{2}$, etc.). It is typical for absorption, as opposed to loss spectroscopies, even when both techniques are employed to study the same system. A detailed analysis of the problem will be the subject of section 6.2 . 


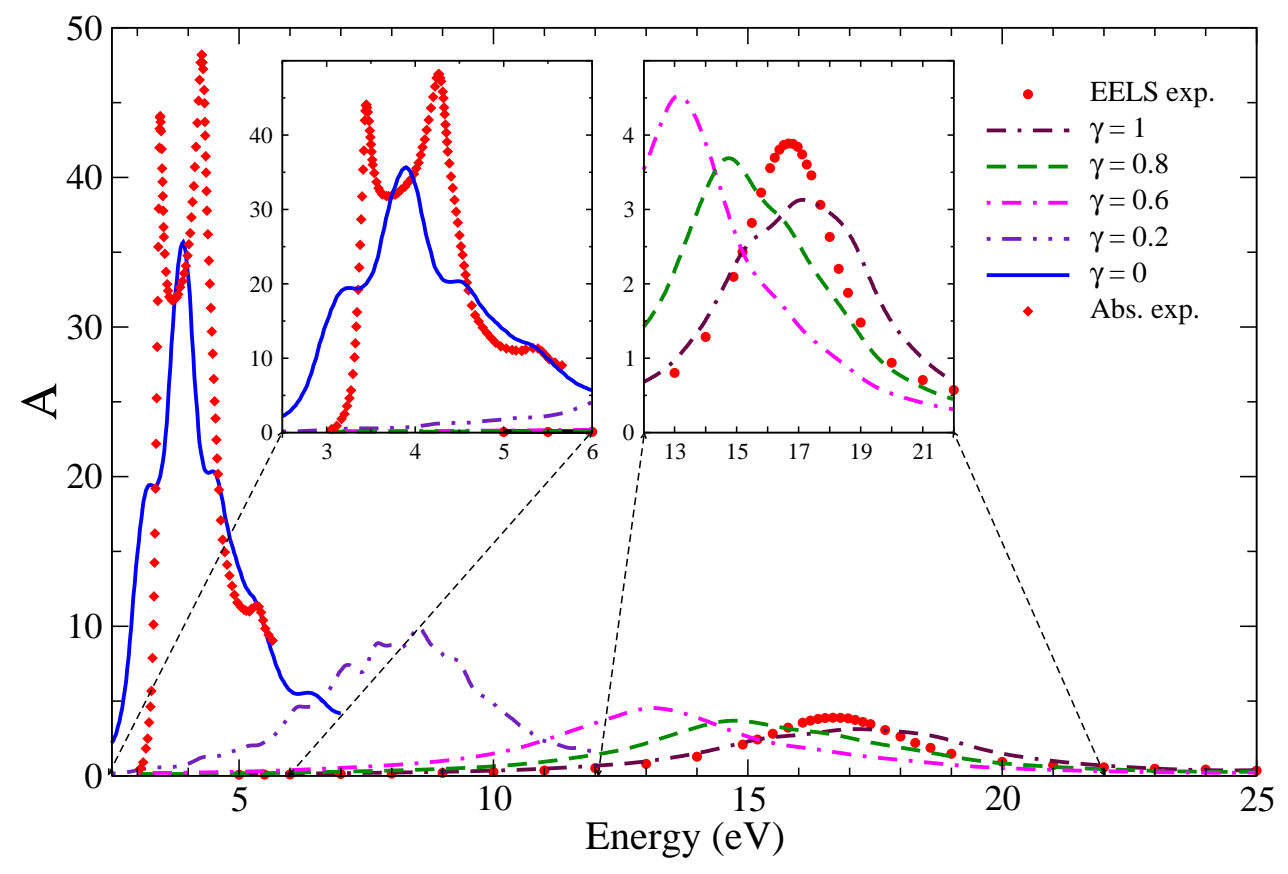

Figure 5. Continuous connection between EELS and absorption spectrum of bulk silicon, via $v_{\mathbf{0}}[66]$. Experiments from references [93] (absorption) and [94] (EELS).

\subsection{EELS and IXSS in extended systems}

In figure 5 we can observe both the absorption and EELS at vanishing $q$ (within RPA) for bulk silicon [66]. To interpret this picture it is useful to use the generalised spectrum $A(\omega)$ of (3.9). The modified RPA polarisation function $X$ (3.9) can be further generalised as

$$
X(\omega)=\left(1-\chi_{\mathrm{KS}} \gamma v_{\mathbf{0}}-\chi_{\mathrm{KS}} \bar{v}\right)^{-1} \chi_{\mathrm{KS}} .
$$

If $\gamma=1, A(\omega)=$ EELS, and if $\gamma=0, A(\omega)=$ Abs. Moreover, it is possible to follow the evolution of the spectrum when $\gamma$ varies continuously from 1 to 0 . Figure 5 shows how the EELS turns continuously into the absorption when $v_{\mathbf{0}}$ is switched off. This exemplifies the action of the long-range component $v_{\mathbf{0}}$, that is responsible for the huge difference between an EELS and an absorption spectrum.

Let us go back to the EEL spectrum of bulk silicon: a comparison between the experimental and the RPA spectrum is shown in the second inset of figure 5 . Olevano and Reining $[95,14]$ showed that the TDLDA gives better agreement with experiment than RPA, even though the difference is small (see figure 16 of [14]). Some improvement can be found when the BSE approach is used (see again figure 16 of [14]). But since the full BSE calculation of a valence plasmon is still a computationally involved task, the use of TDLDA (or even RPA) is often well justified.

When the electron density does not present particular inhomogeneities, it can be enough to include only $v_{\mathbf{0}}$ in the kernel $(2.35)$ to obtain an accurate calculation of the loss spectra of extended systems. In the case of layered or low dimensional structures [96], or in presence of localised states [97, 98], also the contribution of $\bar{v}$ becomes essential and only a RPA, or often better TDLDA, calculation can yield a good agreement with the experimental data. 


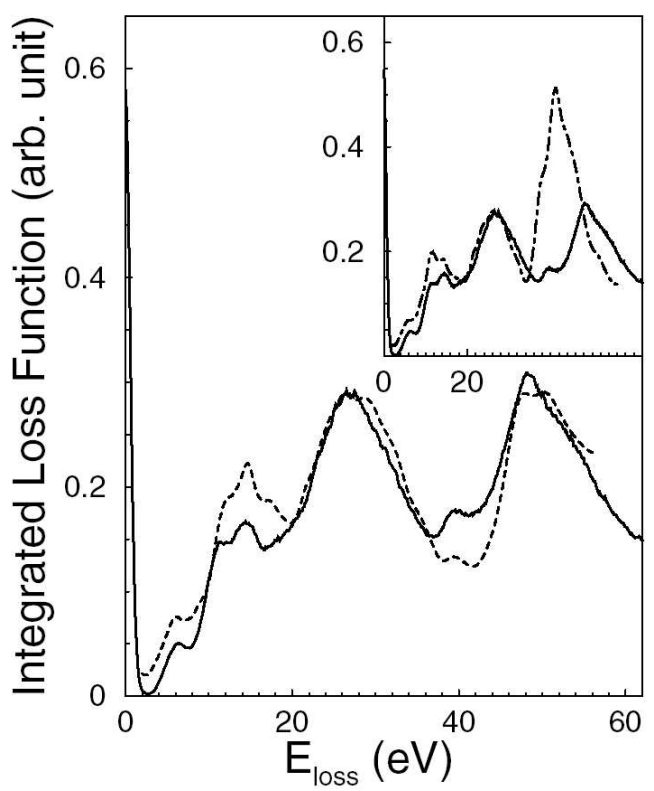

Figure 6. Integrated loss function of $\mathrm{TiO}_{2}$ for $q \simeq 0.4 \AA^{-1}$ )[97]. Solid line: Experiment. Dashed line: RPA calculation. The inset compares the IPA calculations (dashed line) with experiment (solid line).

The similarity between RPA and TDLDA is a quite general feature for the loss function at small transferred momentum q. It holds, e.g., for the loss function of graphite [96], and for the integrated loss function of $\mathrm{TiO}_{2}$ [97]. A good agreement with experimental spectra was obtained within RPA also for the EELS of diamond [27] and $\mathrm{ZrO}_{2}$ [98], always at low momentum transfer. As a general rule, when q gets larger, the contributions of LFE and of the exchange-correlation kernel within TDLDA become more important. Also in this case RPA and TDLDA allow a good agreement with experiment, as it is shown by recent calculations of IXSS at the RPA and TDLDA level for Al [25], rutile $\mathrm{TiO}_{2}$ [75] and various $3 d$ transition metals [26]. In some cases, TDLDA can give a sizable improvement with respect to RPA [28].

The case of $\mathrm{TiO}_{2}$, illustrated in figure 6, is particularly interesting: at small momentum transfer the IPA spectrum yields results up to about $25 \mathrm{eV}$ very similar to those obtained within the RPA or the TDLDA. At higher energies the IPA picture breaks down as it cannot describe correctly the structure originated by transitions from the localised semi-core states. The agreement with the experimental data is obtained only within RPA and TDLDA (the TDLDA spectrum is very similar to the RPA one and therefore it is not shown in figure 6).

These statements often hold when the momentum transfer is small. For large momentum transfer, the LFE become more important and, with the increasing contribution of $\mathbf{G} \neq 0$ terms also the influence of the TDLDA kernel increases. In fact, by comparing IPA, RPA and TDLDA calculations of IXSS for $\mathrm{TiO}_{2}$, Gurtubay et al [75] proved that at large momentum transfer the calculations agree with the experiment only when LFE are included, even at low 


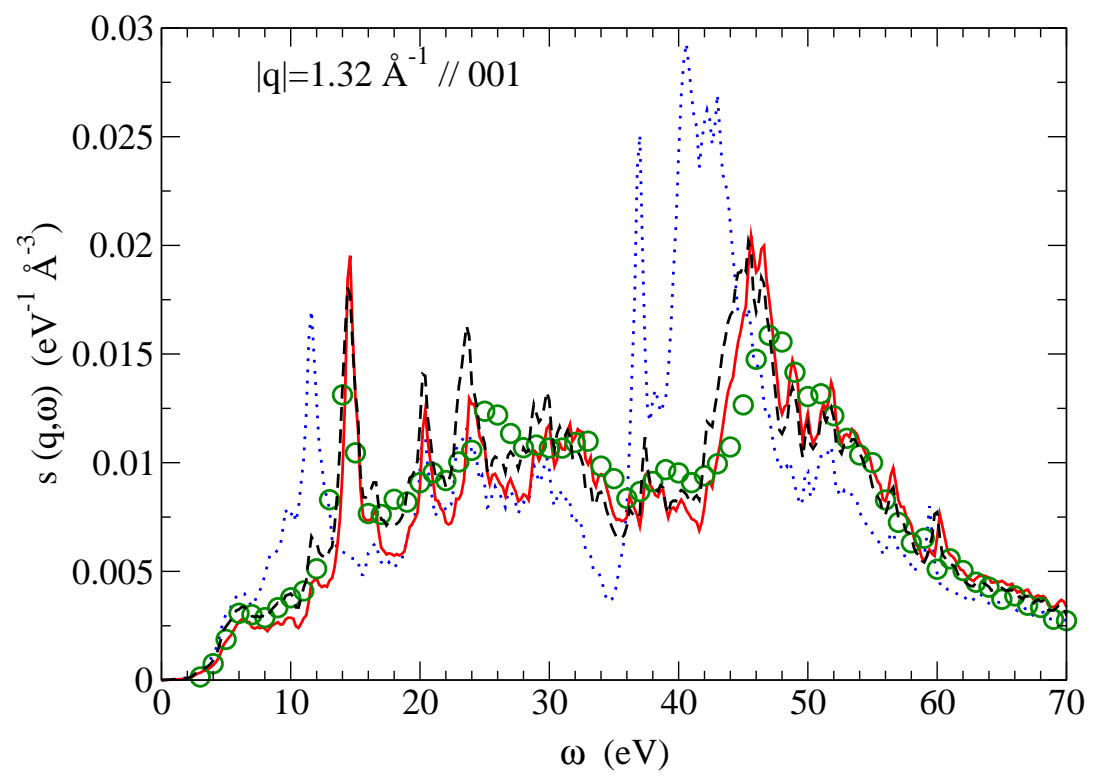

Figure 7. Dynamical structure factor of $\mathrm{TiO}_{2}$ at $|\mathbf{q}|=1.32 \AA^{-1}$ along the [001] direction [75]. Solid (dotted) line: TDLDA spectrum with (without) LFE. Dashed line: RPA spectrum with LFE. Circles: IXS measurements [75].

energies (see figure 7 ).

In conclusion, we can state that the TDLDA is often very reliable for EELS and IXSS (both for small and large momentum transfer), and for photoabsorption in finite systems. LFE often give a sizable contribution to this success. One of the main remaining problems is the optical absorption in extended systems.

\subsection{What is missing in RPA and TDLDA?}

We can summarise now the situation as it was explored so far:

- For excitation properties of finite systems, in general, RPA and TDLDA work quite well. There are of course many exceptions, most of which are related to the incorrect tail of the LDA (or GGA) exchange-correlation potential at large $r$. Some problems related to this deficiency are the already mentioned impossibility to reproduce Rydberg series, the overestimation of polarizabilities in long chain molecules, the large underestimation of ionisation energies or the wrong description of any situation where the electrons are pushed to regions far away from the nuclei (e.g. by a strong laser). These issues can be solved by the use of functionals with the correct asymptotic behaviour, like the EXX or the adiabatic LB94. [99].

- For extended systems, EELS and IXSS at small and large momentum transfer are often well reproduced within TDLDA. Instead, TDLDA fails in the calculation of optical $(q=0)$ spectra of non-metallic solids [23]. To explain this failure, the wrong asymptotic behaviour of the exchange-correlation potential is less relevant, while the wrong asymptotic limit of the exchange-correlation kernel is crucial. For infinite systems, the $q=0$ component of $\chi_{\mathrm{KS}}$ vanishes as $q^{2}$. It is then clear from the response equation (3.7) that if $f_{\mathrm{xc}}$ has to correct the non-interacting response for $q \rightarrow 0$ it will have 
to contain a term that behaves asymptotically as $1 / q^{2}$ when $q \rightarrow 0$. This term will be particularly important in absorption calculations, where the Coulomb part of the kernel does not contain $v_{\mathbf{0}} \sim 1 / q^{2}$. This crucial term cannot be found in the local or gradient-corrected approximations.

Finally, we recall that the TDLDA exchange-correlation potential is local in time. Few attempts to derive functionals which are nonlocal in time, i.e. that include memory effects, have been done so far. By analogy with hydrodynamics, Dobson et al assumed that in the electron liquid memory resides not with each fixed point $\mathbf{r}$, but rather within each separate "fluid element" [100]. Thus the element which arrives at location $\mathbf{r}$ at time $t$ "remembers" what happened to it at earlier times when it was at locations different from its present location r. Using this concept, Dobson et al proposed a functional that satisfies Galilean invariance and Ehrenfest's theorem. Unfortunately, no applications of this functional exist to date. This approach was further extended by Tokatly within time-dependent current DFT [101]. Furthermore, the frequency dependence of the exchange-correlation kernel has been proved to be essential to describe charge transfer between open-shell species [102] and double excitations[103, 104, 105, 106, 107]. An example of frequency dependent model exchangecorrelation kernel will be presented in section 8.1.

Several attempts have been done to correct shortcomings of RPA and TDLDA. In the next section we will start by considering the case of metallic systems and discuss explicit density functional beyond the ALDA.

\section{Explicit density functionals}

Explicit density functionals are expressions for the exchange-correlation kernel that are defined directly in terms of the electron density. Most of the commonly used approximations, such as the ALDA, belong to this class of functionals. The majority is derived from numerical results for the homogeneous electron gas that are cast into a parametrised form, thus allowing a transfer to other systems. The prevalence of explicit density functionals is largely due to the fact that their evaluation is typically cheap and adds little computational overhead to practical calculations. However, the physical content of a given parametrisation, especially when transferred to a very different material, and its accuracy for the study of excited states are not always clear. Some problems of the RPA and the ALDA were already discussed in the previous section. In the following we consider a wider range of explicit density functionals that go beyond these basic approximations and analyse their performance for extended systems.

\subsection{Dynamic exchange-correlation effects in the electron gas}

In the following we examine the influence of the exchange-correlation kernel on the excitation spectrum of the homogeneous electron gas, based on [108]. As the effective ground-state potential is a trivial constant for this model system, the choice of the kernel is the only source of errors, whose impact can thus be clearly identified. In addition, the relative simplicity of the homogeneous electron gas makes it possible to explore accurate numerical constructions that go significantly beyond basic approximations like the RPA and ALDA. Note however that their applicability to inhomogeneous semiconductors with a finite band gap, which most of this review is concerned with, is an entirely different question, because the electronic properties of metallic and non-metallic materials deviate fundamentally. Specifically, we here consider the following schemes. 
(a) In the RPA (3.10) all dynamic exchange and correlation effects are ignored by setting the kernel to zero.

(b) The ALDA replaces the wavevector- and frequency-dependent kernel of the electron gas by its long-wave-length and static limit (for inhomogeneous systems, this value is then used at each point in space according to the local density, see (3.12)):

$$
f_{\mathrm{xc}}^{\mathrm{ALDA}}=\lim _{q \rightarrow 0} f_{\mathrm{xc}}^{\mathrm{HEG}}(q, \omega=0) .
$$

Note that for the homogeneous gas the exact kernel in reciprocal space is of course not a matrix but has only a scalar dependence on the absolute value of $q$. Also note that, contrary to the case of semiconductors and insulators mentioned in earlier sections, no $1 / q^{2}$ divergence appears.

(c) In their original application of TDDFT to excited states Petersilka, Gossmann and Gross (PGG) [20] derived an exchange-only kernel within the approximation of Krieger, Li and Iafrate [109]. Designed for small atoms, the PGG formula is, in fact, exact for twoelectron exchange, but deviations are expected for extended systems. In particular, it does not contain the frequency dependence of the exact exchange kernel [110]. The formula for the non-local PGG kernel $f_{\mathrm{xc}}^{\mathrm{PGG}}(q)$ in reciprocal space for the homogeneous electron gas is given in [111].

(d) Burke, Petersilka and Gross (BPG) [112] proposed a hybrid formula that improves further the excitation spectra of atoms by incorporating correlation as well as a selfinteraction correction. It combines expressions for symmetric and antisymmetric spin orientations from different approximations in a spin density-functional formalism. For the unpolarised homogeneous electron gas this kernel reduces to

$$
f_{\mathrm{xc}}^{\mathrm{BPG}}(q)=\frac{1}{2}\left[f_{\mathrm{xc}, \uparrow \uparrow}^{\mathrm{PGG}}(q)+f_{\mathrm{xc}, \uparrow \downarrow}^{\mathrm{ALDA}}\right]
$$

(e) A good parametrisation of the static exchange-correlation kernel for the homogeneous electron gas was given by Corradini, Del Sole, Onida and Palummo [113] (CDOP), who used the Monte Carlo results of Moroni, Ceperley and Senatore [114] for the static local-field factor $G(q)=-f_{\mathrm{xc}}(q) / v(q)$. Unlike the original data, this parametrisation is not restricted to metallic densities, because it incorporates the known asymptotic limits for high and low densities. By construction, the CDOP kernel becomes identical to the ALDA in the long wave-length limit.

(f) Finally, we consider a parametrisation of the dynamic local-field factor of the homogeneous electron gas proposed by Richardson and Ashcroft [115] (RA), including the corrections given in [111], which stems from the summation of self-energy, exchange and fluctuation terms in the diagrammatic expansion of the polarisation function. It satisfies many important sum rules and reproduces the exact asymptotic expressions for small and large wave vectors. At intermediate wave vectors and frequencies it provides a realistic description of the position and magnitude of extrema, which are related to the pair distribution function evaluated at zero separation. Because of this careful derivation one can expect the RA expression to be very close to the exact dynamic exchange-correlation kernel of the homogeneous electron gas and to give an accurate account of the plasmon dispersion. In the absence of experimental data we therefore use the RA results as a reference in order to assess the performance of simpler approximations. The parametrisation was originally given on the imaginary frequency axis; here we use its continuation to the full complex plane.

Among the many other expressions with practical relevance we mention the early parameterisations of the static local-field factor by Hubbard [116], Vashishta and Singwi 
$[117,118]$ and Utsumi and Ichimaru [119], which are now superseded by more accurate Monte Carlo results, and an attempt by Gross and Kohn [17] to retain the frequency dependence within the local-density approximation at long wave lengths. Unfortunately, the latter violates a number of exact conditions, especially the harmonic-potential theorem [120]. The dynamic and non-local exact exchange kernel, an implicit density functional constructed from the Kohn-Sham wave functions, as well as other orbital dependent functionals, are discussed later in section 5 .

The poles of the linear density-response function of the homogeneous electron gas

$$
\chi(q, \omega)=\frac{\chi_{\mathrm{KS}}(q, \omega)}{1-\chi_{\mathrm{KS}}(q, \omega)\left[v(q)+f_{\mathrm{xc}}(q, \omega)\right]}
$$

stem from two different sources. The singularities of the Kohn-Sham density-response function in the numerator correspond to independent electron-hole pair excitations; they form a continuum that is bounded by the lines $\frac{1}{2} q^{2}-q k_{\mathrm{F}} \leq \omega \leq \frac{1}{2} q^{2}+q k_{\mathrm{F}}$. In addition, the zeroes of the denominator give rise to a distinct plasmon branch $\omega_{q}$, which describes resonant collective charge oscillations of the electron system. We focus on the latter, since the plasmon dispersion gives a direct measure for the quality of the kernel. Before scrutinising the numerical results we first discuss what can be deduced from an analytic expansion of the plasmon dispersion up to second order in $q[121]$ :

$$
\omega_{q}=\omega_{\mathrm{pl}}\left[1+\left(\frac{9}{10 k_{\mathrm{TF}}^{2}}+\frac{f_{\mathrm{xc}}\left(0, \omega_{\mathrm{pl}}\right)}{8 \pi}\right) q^{2}+\mathrm{O}\left(q^{4}\right)\right],
$$

where $k_{\mathrm{TF}}=2(3 n / \pi)^{1 / 6}$ is the Thomas-Fermi wave vector. As none of the above $f_{\mathrm{xc}}$ diverges, all curves approach the classical plasma frequency $\omega_{\mathrm{pl}}=(4 \pi n)^{1 / 2}$ in the long wave-length limit. The kernel only introduces corrections in second order, where the element $f_{\mathrm{xc}}\left(0, \omega_{\mathrm{pl}}\right)$ appears. The ALDA contains by construction (4.1) the correct long wave-length limit, but its neglect of the frequency dependence introduces an error in the parabolic term. The CDOP formula produces the same second-order term as the ALDA since the two kernels, that are both static, coincide for $\lim _{q \rightarrow 0}$. Also the PGG and BPG functionals are static approximations; moreover they do not approach the correct long wave-length limit of the homogeneous electron gas and therefore generate a different parabolic coefficient. The RA kernel, which incorporates the full frequency dependence, is the only parametrisation that is formally exact beyond the trivial zeroth order.

The calculated plasmon dispersions for $r_{\mathrm{s}}=4$, obtained from a numerical search for the zeroes of the denominator of (4.3) in the complex frequency plane, are shown in figure 8 . The results are representative for the whole range of metallic densities [108]. As predicted, all curves start at the classical plasma frequency. For small wave vectors only a minor spread of the results is observed, because the factor $9 /\left(10 k_{\mathrm{TF}}^{2}\right)$ in $(4.4)$ outweighs the contribution of the kernel. However, a slight downward shift compared to the RPA is clearly visible for all non-trivial approximations, because dynamic exchange and correlation effects combine to lower the energy of the electron system. The ALDA and the CDOP formula produce curves that are initially very close to the reference RA result, indicating that the neglected frequency dependence is of little consequence as long as the correct long wave-length limit is reproduced. This point is emphasised further by the relatively large deviation for the static PGG kernel, which stems precisely from its incorrect behaviour at $q \rightarrow 0$. The BPG curve, as expected, lies between the ALDA and PGG results.

At larger wave vectors, where the parabolic expansion (4.4) is no longer valid, the differences between the considered approximations become more pronounced. The dispersion 


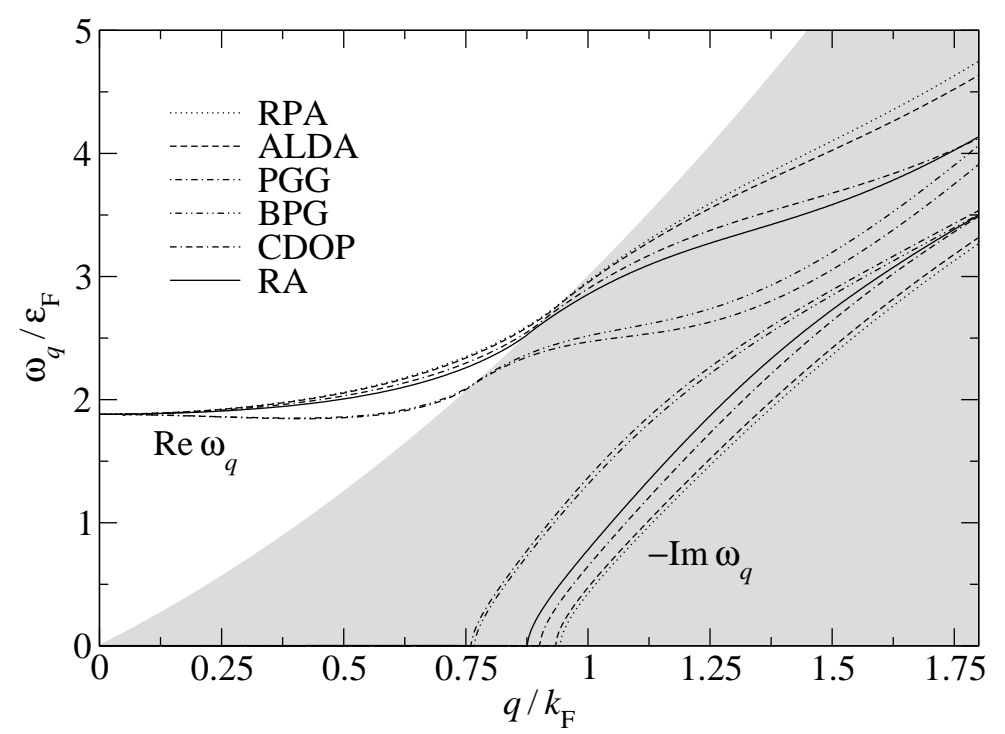

Figure 8. Plasmon dispersion for the homogeneous electron gas at $r_{\mathrm{s}}=4$ calculated with different approximations for the exchange-correlation kernel (see text). The grey-shaded area marks the electron-hole pair continuum. The finite imaginary part of the plasmon energy in this region is also shown (see also [108]).

resulting from the static ALDA kernel remains close to the RPA and yields too high energies, while the CDOP result begins to deviate slightly from the RA curve after the onset of damping in the electron-hole pair continuum. This discrepancy must be attributed to the static nature of the CDOP kernel. Furthermore, it can be seen that the strong downward shift of the exchange-only PGG formula leads to an even larger error in absolute terms than the underestimation of dynamic exchange and correlation effects in both the ALDA and the RPA. The hybrid BPG formula, which combines the PGG and ALDA parameterisations, profits from a partial cancellation of errors but improves only marginally upon PGG.

Due to the possible decay into electron-hole pairs, the plasmon energy contains a non-zero imaginary part, also displayed in figure 8 , whose inverse is proportional to the excitation lifetime. As a general rule, all kernels yield the same quality of approximation for the imaginary part as for the real part of the plasmon energy. At small wave vectors all static kernels predict a vanishing imaginary part, which corresponds to an unphysical infinite lifetime. This artifact results from modelling $f_{\mathrm{xc}}(q, \omega)$ as a purely real quantity by evaluating it at $\omega=0$. In contrast, the exact kernel has a finite imaginary part at non-zero frequencies, which for small wave vectors is related to the multi-pair component of the linear densityresponse function [122]. Such multi-pair decay channels are ignored in the RPA and related schemes, which is ultimately the reason for their qualitatively wrong behaviour. Mermin's modification of the Lindhard dielectric function avoids the problem of infinite lifetimes [123], but the correction based on relaxation times is introduced in a phenomenological manner that makes it unsuitable for ab initio calculations. Among the expressions considered here, only the dynamic RA parametrisation correctly predicts a finite plasmon lifetime over the entire frequency range, although outside the electron-hole pair continuum the imaginary part of the plasmon energy is several orders of magnitude smaller than the real part and not discernible in the figure.

The good agreement between the static CDOP parametrisation on the one hand 
and the dynamic RA result on the other over a large wave-vector and density interval indicates that the frequency dependence of the kernel plays a weak role for the plasmon dispersion in the homogeneous electron gas. In contrast, the significant discrepancy between static approximations like the ALDA that contain the correct long wave-length limit and others, such as PGG, which do not, suggests that a correct parametrisation of the wavevector dependence is crucial. Similar conclusions concerning the relative importance of the frequency and wave-vector dependence were also reported for the correlation energy of the homogeneous electron gas [111].

\subsection{Extension to inhomogeneous systems}

As the homogeneous electron gas allows a highly accurate treatment of the kernel that includes the full wave-vector and frequency dependence, it is tempting to transfer these results to real inhomogeneous systems. Such an approach is mathematically justified if the variation of the density $n_{G S}(\mathbf{r})=\bar{n}+\Delta n(\mathbf{r})$ with $\int \Delta n(\mathbf{r}) \mathrm{d}^{3} r=0$ from a homogeneous charge distribution $\bar{n}$ is small, i.e., $|\Delta n(\mathbf{r}) / \bar{n}| \ll 1$. In this case the kernel can safely be approximated as [3]

$$
f_{\mathrm{xc}}\left(\mathbf{r}, \mathbf{r}^{\prime}, \omega\right) \approx f_{\mathrm{xc}}^{\mathrm{HEG}}\left(\bar{n},\left|\mathbf{r}-\mathbf{r}^{\prime}\right|, \omega\right) .
$$

It should be noted that rapid density oscillations leading to large gradients are not explicitly excluded, as long as the magnitude of the oscillations themselves remains sufficiently small.

Unfortunately, the approximation (4.5) is of rather little practical use, because the condition of an almost constant electron density is almost never fulfilled for real materials. However, for $\omega=0$ a very similar expression with a much wider range of validity can also be obtained under less restrictive assumptions. The derivation is based on the observation that the static exchange-correlation kernel, although non-local, is actually short-ranged and decays rapidly if the separation $\left|\mathbf{r}-\mathbf{r}^{\prime}\right|$ significantly exceeds the inverse Fermi wave vector. Furthermore, it depends only on the electronic structure in the vicinity of the points $\mathbf{r}$ and $\mathbf{r}^{\prime}$. This is a manifestation of the "nearsightedness" principle [124] and caused by the destructive interference of wave functions in quantum-mechanical many-particle systems over a typical de Broglie wave length. The nearsightedness principle is strictly valid only in equilibrium and hence does not apply to the dynamic exchange-correlation kernel at non-zero frequencies. Therefore, it fails to cover spectroscopies like EELS or optical absorption that include a finite energy transfer, but the static kernel may be useful in other situations, such as total-energy calculations [3, 125].

In combination with the short range of the static exchange-correlation kernel, the nearsightedness principle implies that the approximation [3, 125]

$$
f_{\mathrm{xc}}\left(\mathbf{r}, \mathbf{r}^{\prime}, 0\right) \approx f_{\mathrm{xc}}^{\mathrm{HEG}}\left(\bar{n}\left(\mathbf{r}, \mathbf{r}^{\prime}\right),\left|\mathbf{r}-\mathbf{r}^{\prime}\right|, 0\right)
$$

is valid if the density variation is small on a length scale given by a few inverse Fermi wave vectors. The definition of the average density $\bar{n}\left(\mathbf{r}, \mathbf{r}^{\prime}\right)$ is arbitrary from a mathematical point of view; as long as it reduces to $\bar{n}=n_{G S}(\mathbf{r})=n_{G S}\left(\mathbf{r}^{\prime}\right)$ in the limit of a homogeneous density distribution, the expression (4.6) is exact to zeroth order. It is reasonable to expect, however, that a suitable choice might improve the performance of the functional for realistic inhomogeneities. Indeed, it has been demonstrated that some possible and, at first sight, plausible definitions like the mid-point density $\bar{n}\left(\mathbf{r}, \mathbf{r}^{\prime}\right)=n_{G S}\left(\frac{1}{2}\left(\mathbf{r}+\mathbf{r}^{\prime}\right)\right)$ can even lead to divergences in total-energy calculations for strongly inhomogeneous systems, such as atoms and surfaces [126]. The reason for the observed unphysical behaviour is that $\bar{n}\left(\mathbf{r}, \mathbf{r}^{\prime}\right)$ approaches zero in this case if one spatial argument moves far into the vacuum 
region while the other remains inside the system. As a consequence, the corresponding Fermi wave vector also approaches zero, and the kernel becomes long-ranged, so that spatial integrations pick up many incorrect contributions. The problem is avoided by the choice $\bar{n}\left(\mathbf{r}, \mathbf{r}^{\prime}\right)=\frac{1}{2}\left[n_{G S}(\mathbf{r})+n_{G S}\left(\mathbf{r}^{\prime}\right)\right]$, which guarantees a finite density and Fermi wave vector in the same situation and is thus recommended. It can indeed be argued to constitute the most logical choice, because it represents a smooth function by its value at some average point of its arguments (Weierstraß), not of the parametrisation of its arguments [125].

Finally, it should be borne in mind that the approximation (4.6) is still derived by an expansion starting from the homogeneous electron gas. Therefore, its validity is restricted to systems where perturbation theory is applicable, i.e., where the inhomogeneity of the density does not alter the physical properties of the system qualitatively. This includes, for example, many bulk metals. On the other hand, it fails to improve the optical absorption spectra of semiconductors, like silicon [127], because the Fourier transform of (4.6) tends to the finite value of the ALDA kernel in the long wave-length limit. Strictly speaking, of course, the restriction of the nearsightedness principle to static phenomena already precludes the use of (4.6) from the outset in this case.

\section{Orbital-dependent functionals}

In contrast to explicit density functionals, orbital-dependent functionals are constructed from the Kohn-Sham wave functions (of course they still depend implicitly on the electron density through the self-consistency condition). Although the computational cost is typically much higher than the straightforward evaluation of a parametrisation in terms of the density, this approach has the advantage that it offers a systematic route to successively more accurate approximations. Orbital-dependent functionals can be obtained in various ways. One can get a potential and kernel from a functional derivative of a suitable approximation of the action, for example by expanding the latter in powers of the Coulomb interaction; this is outlined in subsection 5.2 below. Alternatively, one can exploit links between many-body perturbation theory (MBPT) and the density-functional formulation. In the next subsection we briefly show how known results can be obtained from a linearised version of this link; section 6 is dedicated to a more detailed discussion of this connection, including results that do not involve a linearisation.

\subsection{Potentials and kernels from a linearised Sham-Schlüter equation}

The density-functional and the MBPT framework are linked by the requirement that the former has to yield the correct density given by the one-particle Green's function $G$ of the latter, via $n(\mathbf{r}, t)=-i G\left(\mathbf{r}, \mathbf{r}^{\prime}, t, t^{+}\right)$, for the static as well as for the time-dependent case. (Note that in the latter case it is recommended to use Keldysh Green's functions in order to obtain the physical densities via the simple relation above [128]). From the Dyson equation one obtains hence

$$
0=\int d 2 d 3 G_{\mathrm{KS}}(1,2)\left(\Sigma(2,3)-v_{\mathrm{xc}}(2) \delta(2,3)\right) G\left(3,1^{+}\right),
$$

where a number " 1 " represent position, spin and time $\left(\mathbf{r}_{1}, \sigma_{1}, t_{1}\right)$ and $\Sigma$ is the self-energy $[129,130]$. This is the so-called Sham-Schlüter equation $[131,128]$. If $G$ is replaced by $G_{\mathrm{KS}}$ everywhere (including in the construction of $\Sigma$, as symbolised by $\Sigma^{\mathrm{KS}}$ ) the solution of this linearised equation yields for the potential 


$$
v_{\mathrm{xc}}(1)=-i \int d 2 d 3 \int d 4 \chi_{\mathrm{KS}}^{-1}(1,2) G_{\mathrm{KS}}(2,3) \Sigma^{\mathrm{KS}}(3,4) G_{\mathrm{KS}}\left(4,2^{+}\right) .
$$

The kernel $f_{\mathrm{xc}}$, the functional derivative with respect to the density of $v_{\mathrm{xc}}$, has a contribution $f^{(2)}$ that stems from the derivative of $\Sigma^{K S}$ and other terms $f^{(1)}$ coming from the derivative of the Green's functions and the inverse response function [132]. Both are explicitly orbitaldependent.

As an example, one can use the simplest case where $\Sigma^{K S}$ is approximated by the (KohnSham) Fock operator $\Sigma_{\mathrm{x}}^{\mathrm{KS}}(1,2)=i G_{K S}(1,2) v(2,1)$. In that case, (5.2) yields the so-called exact-exchange (EXX) OEP potential [110, 133]

$$
v_{\mathrm{EXX}}(1)=-i \int d 2 d 3 \int d 4 \chi_{\mathrm{KS}}^{-1}(1,2) G_{\mathrm{KS}}(2,3) \Sigma_{\mathrm{x}}^{\mathrm{KS}}(3,4) G_{\mathrm{KS}}\left(4,2^{+}\right),
$$

where all KS quantities are calculated self-consistently using the EXX potential. The contributions to the kernel become

$$
\begin{aligned}
f^{(2), \operatorname{EXx}}(1,2)=\int d 3 d 4 d 5 d 6 \chi_{K S}^{-1}(1,3) G_{\mathrm{KS}}(3,4) G_{\mathrm{KS}}(5,3) & \\
& \times v(4,5) G_{\mathrm{KS}}(4,6) G_{\mathrm{KS}}(6,5) \chi_{\mathrm{KS}}^{-1}(6,2)
\end{aligned}
$$

(which is nothing else but the electron-hole attraction term of time-dependent-EXX, TD$\operatorname{EXX}[50,133,132])$. The rest of the terms - which has the difficult task to open the bandgap with respect to the KS one in TD-EXX - reads

$$
\begin{aligned}
& f^{(1), \operatorname{EXX}}(1,2)=\int d 3 d 4 d 5 d 6 \chi_{\mathrm{KS}}^{-1}(1,3) G_{\mathrm{KS}}(3,6) G_{\mathrm{KS}}(6,4) \\
& \times\left[\Sigma_{x}^{\mathrm{KS}}(4,5)-\delta(4,5) v_{\mathrm{KS}}(4)\right] G_{\mathrm{KS}}(5,3) \chi_{\mathrm{KS}}^{-1}(6,2) \\
& +\int d 3 d 4 d 5 d 6 \chi_{\mathrm{KS}}^{-1}(1,3) G_{\mathrm{KS}}(6,3) G_{\mathrm{KS}}(3,4) \\
& \times\left[\Sigma_{x}^{\mathrm{KS}}(4,5)-\delta(4,5) v_{\mathrm{KS}}(4)\right] G_{\mathrm{KS}}(5,6) \chi_{K S}^{-1}(6,2) .
\end{aligned}
$$

TD-EXX will be discussed more in detail in the next subsection.

\subsection{The time-dependent optimised-potential method from an action formalism}

The TD-OEP potential can also be obtained from the action formalism. In this case, the time-dependent optimised-potential method [49] treats the Coulomb interaction as a perturbation that is switched on adiabatically in the interval $\left(-\infty, t_{0}\right)$, while a compensating local potential ensures that the density remains constant and equal to the static groundstate density $n_{\mathrm{GS}}(\mathbf{r})$ throughout the entire switching-on process. In this way it provides an adiabatic connection between the stationary ground state of the non-interacting Kohn-Sham system at $t \rightarrow-\infty$ and the wave function $\Psi_{0}$ of the true interacting electrons at $t=t_{0}$ that enters in the definition of the action (2.21). In order to incorporate the switching-on process in the theoretical description of the evolution of the system, the beginning and end of the pseudotime contour $C$ must be extended to $-\infty$. The combination of the adiabatic connection with the time-contour method makes it possible to apply standard perturbation techniques and expand $A_{\mathrm{xc}}[n]$ in terms of the Kohn-Sham orbitals and the Coulomb interaction [128]. 
For an orbital-dependent action, two equivalent expressions for $\delta A_{\mathrm{xc}}[n] / \delta v_{\mathrm{KS}}(\mathbf{r}, \tau)$ can be derived by applying the chain rule with different intermediate quantities:

$$
\begin{aligned}
& \int_{C} d \tau^{\prime} \int d^{3} r^{\prime} \frac{\delta A_{\mathrm{xc}}[n]}{\delta n\left(\mathbf{r}^{\prime}, \tau^{\prime}\right)} \frac{\delta n\left(\mathbf{r}^{\prime}, \tau^{\prime}\right)}{\delta v_{\mathrm{KS}}(\mathbf{r}, \tau)} \\
&=\int_{C} d \tau^{\prime} \int d^{3} r^{\prime} \sum_{j=1}^{\infty}\left(\frac{\delta A_{\mathrm{xc}}[n]}{\delta \varphi_{j}\left(\mathbf{r}^{\prime}, \tau^{\prime}\right)} \frac{\delta \varphi_{j}\left(\mathbf{r}^{\prime}, \tau^{\prime}\right)}{\delta v_{\mathrm{KS}}(\mathbf{r}, \tau)}+\frac{\delta A_{\mathrm{xc}}\left[\varphi_{j}\right]}{\delta \varphi_{j}^{*}\left(\mathbf{r}^{\prime}, \tau^{\prime}\right)} \frac{\delta \varphi_{j}^{*}\left(\mathbf{r}^{\prime}, \tau^{\prime}\right)}{\delta v_{\mathrm{KS}}(\mathbf{r}, \tau)}\right) .
\end{aligned}
$$

The first functional derivative on the left-hand side gives rise to the exchange-correlation potential $(2.25)$, which can be determined from this identity because all other terms are known: The linear Kohn-Sham density-response function $\delta n\left(\mathbf{r}^{\prime}, \tau^{\prime}\right) / \delta v_{\mathrm{KS}}(\mathbf{r}, \tau)$ is obtained with the help of time-dependent perturbation theory by applying the same techniques as in section 2 in the pseudotime domain, while the derivatives of $A_{\mathrm{xc}}[n]$ on the right-hand side of (5.6) can be calculated analytically for a given orbital-dependent functional. The variations of the orbitals

$$
\frac{\delta \varphi_{j}\left(\mathbf{r}^{\prime}, \tau^{\prime}\right)}{\delta v_{\mathrm{KS}}(\mathbf{r}, \tau)}=-\mathrm{i} \varphi_{j}(\mathbf{r}, \tau) \sum_{k=1}^{\infty} \varphi_{k}^{*}(\mathbf{r}, \tau) \varphi_{k}\left(\mathbf{r}^{\prime}, \tau^{\prime}\right) \Theta\left(\tau^{\prime}-\tau\right)
$$

and the corresponding formulas for the conjugate orbitals $\delta \varphi_{j}^{*}\left(\mathbf{r}^{\prime}, \tau^{\prime}\right) / \delta v_{\mathrm{KS}}(\mathbf{r}, \tau)$ again follow from time-dependent perturbation theory. Finally, an expression for the exchange-correlation kernel is obtained by manipulating in an analogous way the second functional derivative

$$
\begin{aligned}
& \frac{\delta^{2} A_{\mathrm{xc}}[n]}{\delta v_{\mathrm{KS}}(\mathbf{r}, \tau) \delta v_{\mathrm{KS}}\left(\mathbf{r}_{2}, \tau_{2}\right)}=\int C d \tau^{\prime} \mathrm{d} \tau_{1} \int \mathrm{d}^{3} r^{\prime} \mathrm{d}^{3} r_{1} \frac{\delta^{2} A_{\mathrm{xc}}[n]}{\delta n\left(\mathbf{r}^{\prime}, \tau^{\prime}\right) \delta n\left(\mathbf{r}_{1}, \tau_{1}\right)} \frac{\delta n\left(\mathbf{r}^{\prime}, \tau^{\prime}\right)}{\delta v_{\mathrm{KS}}(\mathbf{r}, \tau)} \frac{\delta n\left(\mathbf{r}_{1}, \tau_{1}\right)}{\delta v_{\mathrm{KS}}\left(\mathbf{r}_{2}, \tau_{2}\right)} \\
&+\int C \mathrm{~d} \tau^{\prime} \int \mathrm{d}^{3} r^{\prime} \frac{\delta A_{\mathrm{xc}}[n]}{\delta n\left(\mathbf{r}^{\prime}, \tau^{\prime}\right)} \frac{\delta^{2} n\left(\mathbf{r}^{\prime}, \tau^{\prime}\right)}{\delta v_{\mathrm{KS}}(\mathbf{r}, \tau) \delta v_{\mathrm{KS}}\left(\mathbf{r}_{2}, \tau_{2}\right)} ; \quad \text { (5.8) }
\end{aligned}
$$

here the term on the right side containing the double derivative of $A_{\mathrm{xc}}[n]$ is nothing else but $\chi_{\mathrm{KS}} f_{\mathrm{xc}} \chi_{\mathrm{KS}}$, which allows one to solve for $f_{\mathrm{xc}}$ after evaluation of all other terms.

\subsection{Exact exchange}

The leading term in the expansion of $A_{\mathrm{xc}}[n]$ in powers of the Coulomb interaction is the exchange part, which is of first order and given by

$$
A_{\mathrm{x}}[n]=-\frac{1}{2} \int C d \tau \sum_{j=1}^{\infty} f_{j} \sum_{k=1}^{\infty} f_{k} \int d^{3} r \int d^{3} r^{\prime} \frac{\varphi_{j}^{*}(\mathbf{r}, \tau) \varphi_{k}(\mathbf{r}, \tau) \varphi_{j}\left(\mathbf{r}^{\prime}, \tau\right) \varphi_{k}^{*}\left(\mathbf{r}^{\prime}, \tau\right)}{\left|\mathbf{r}-\mathbf{r}^{\prime}\right|},
$$

while the correlation part $A_{\mathrm{c}}[n]$ includes all higher-order contributions. Inserting this expression into (5.6) and evaluating all quantities with the static density $n_{\mathrm{GS}}(\mathbf{r})$ on the physical time axis yields the "exact exchange potential" [134, 135], (5.3). For the static ground state potential this yields a relatively simple expression

$$
\begin{aligned}
& \int d^{3} r^{\prime} v_{\mathrm{EXX}}\left(\mathbf{r}^{\prime}\right) \chi_{\mathrm{KS}}\left(\mathbf{r}^{\prime}, \mathbf{r} ; \omega=0\right) \\
& =\int d^{3} r^{\prime} \int d^{3} r^{\prime \prime} \Sigma_{\mathbf{x}}\left(\mathbf{r}^{\prime}, \mathbf{r}^{\prime \prime}\right) \sum_{j=1}^{\infty} \sum_{k=1}^{\infty}\left(f_{j}-f_{k}\right) \frac{\varphi_{j}^{*}\left(\mathbf{r}^{\prime}\right) \varphi_{k}\left(\mathbf{r}^{\prime \prime}\right) \varphi_{j}(\mathbf{r}) \varphi_{k}^{*}(\mathbf{r})}{\varepsilon_{j}-\varepsilon_{k}},
\end{aligned}
$$


with the non-local exchange self-energy

$$
\Sigma_{\mathrm{x}}\left(\mathbf{r}^{\prime}, \mathbf{r}^{\prime \prime}\right)=-\sum_{j=1}^{\infty} f_{j} \frac{\varphi_{j}(\mathbf{r}) \varphi_{j}^{*}\left(\mathbf{r}^{\prime}\right)}{\left|\mathbf{r}-\mathbf{r}^{\prime}\right|}
$$

As pointed out above the latter takes the same form as the Hartree-Fock exchange operator but is here constructed from the Kohn-Sham orbitals. Solving (5.10) for the exact exchange potential requires an inversion of the static linear density-response function $\chi_{\mathrm{KS}}\left(\mathbf{r}^{\prime}, \mathbf{r} ; 0\right)$, which is in practise achieved by a matrix inversion after projection on a suitable basis set, such as plane waves for extended systems $[136,137]$. The matrix elements with reciprocal lattice vectors $\mathbf{G}=\mathbf{0}$ or $\mathbf{G}^{\prime}=\mathbf{0}$ and $\mathbf{q}=\mathbf{0}$ must be omitted in this case [138]. The restriction to this submatrix is necessary to guarantee a one-to-one correspondence between variations of the density and the effective potential and hence to obtain an invertible operator; it excludes constant potential shifts that leave the density unchanged as well as density variations that violate particle-number conservation.

Compared to the evaluation of explicit density functionals, the construction of $v_{\operatorname{EXX}}(\mathbf{r})$ is considerably more expensive, because it requires not only the occupied conduction states but also a summation over the unoccupied part of the spectrum. In addition, the linear density-response function must be inverted in each cycle of the self-consistency loop. Practical calculations were therefore initially restricted to atoms and small molecules, but the method is now also routinely applied to bulk semiconductors, insulators and metals [138, 139, 140, 141, 142, 143, 144].

From a theoretical point of view, the exact exchange potential has definite advantages. In particular, it is free of self-interaction and exhibits the correct asymptotic behaviour outside finite systems [135], where it decays like $-1 / r$, while the LDA and GGA fall off exponentially. The exact exchange potential further features a discontinuity with respect to a change in the number of electrons [143]. Such a discontinuity is also contained in the exact functional; it is the difference between the Kohn-Sham eigenvalue gap and the true quasiparticle band gap in semiconductors [145, 131].

The "exact exchange" kernel is similarly obtained, following the outline of (5.8), from the relation

$$
\int d^{3} r^{\prime \prime} \int d^{3} r^{\prime \prime \prime} \chi_{\mathrm{KS}}\left(\mathbf{r}, \mathbf{r}^{\prime \prime} ; \omega\right) f_{\mathrm{x}}\left(\mathbf{r}^{\prime \prime}, \mathbf{r}^{\prime \prime \prime} ; \omega\right) \chi_{\mathrm{KS}}\left(\mathbf{r}^{\prime \prime \prime}, \mathbf{r}^{\prime} ; \omega\right)=P_{1}\left(\mathbf{r}, \mathbf{r}^{\prime} ; \omega\right)
$$

The right-hand side equals the first-order contribution to the irreducible polarizability in an expansion in powers of the Coulomb interaction. It consists of five distinct terms, which can be derived in matrix notation [110] but are more easily summarised in terms of Feynman diagrams [146]. The diagrammatic form of $P_{1}\left(\mathbf{r}, \mathbf{r}^{\prime} ; \omega\right)$ is shown in figure 9 together with the representation of (5.10). The first two terms are the self-energy insertions with the non-local exchange operator for independent electrons and (contributions due to $\Sigma_{\mathrm{x}}$ in (5.5)), while the third arises from the attractive electron-hole interaction (5.4). The last two terms contain the exact local exchange potential (contributions due to $v_{\mathrm{xc}}$ in (5.5)) and must be subtracted in order to avoid double counting.

In contrast to the ALDA, the exact exchange kernel is both non-local and frequencydependent. Furthermore, in the case of semiconductors its Fourier transform diverges in the limit of small wave vectors, as required for the exact functional [110].

As a first practical test of its performance for electronic excitations in extended systems we display the plasmon dispersion for the homogeneous electron gas at $r_{\mathrm{s}}=4$ calculated with the exact exchange kernel in figure 10. The ALDA and the results obtained with the 
(a)

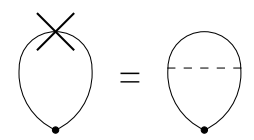

(b)

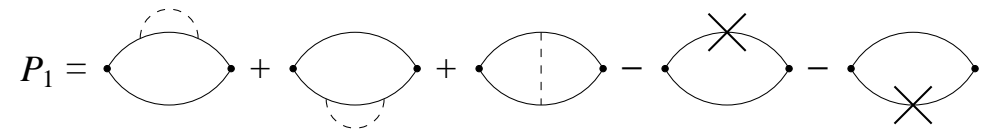

Figure 9. Diagrammatic form of (a) the equation for the exact exchange potential and (b) the first-order irreducible polarizability. A solid line represents the Green function of the non-interacting Kohn-Sham electrons, a broken line represents the Coulomb interaction, and the local exchange potential is indicated by a cross.

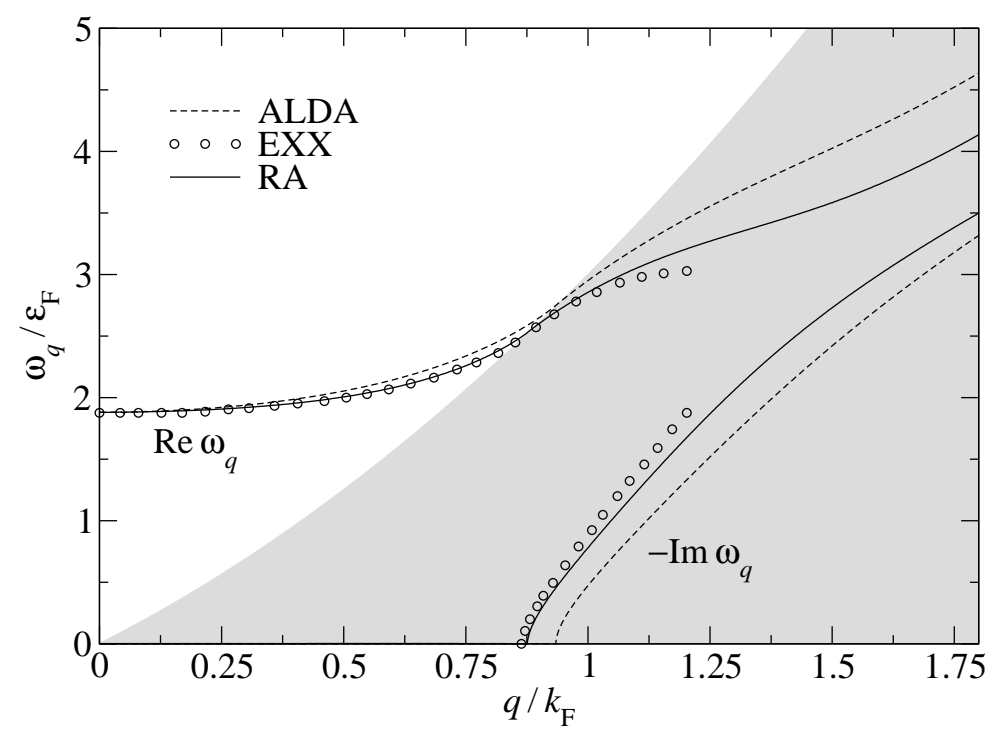

Figure 10. Plasmon dispersion for the homogeneous electron gas at $r_{\mathrm{s}}=4$ with the exact exchange kernel (EXX) compared to the ALDA and the reference values obtained with the parametrisation by Richardson and Ashcroft (RA) [115].

parametrisation by Richardson and Ashcroft [115] are shown for comparison and are the same as in figure 8 above. The exact exchange kernel is evidently in very good agreement with the RA reference values in the entire region outside the electron-hole pair continuum and constitutes a definite improvement over the ALDA. Based on our earlier analysis, we attribute this to a close match with the wave-vector-dependence of the complete functional and conclude that the exact exchange kernel is a suitable starting point for the quantitative investigation of collective excitations in free-electron metals [147].

Early results from the time-dependent exact exchange method for semiconductors indicated a good performance: Kim and Görling [133] calculated the optical absorption spectrum of silicon and found good agreement with experimental data. However, it was later observed that those calculations contained a cutoff of the divergent Coulomb potential at small wave vectors, which actually had a large effect on the results and was in fact responsible for the good quantitative performance [132]. As the singularity reflects the 
long range of the Coulomb interaction in real space, this cutoff means that the kernel was effectively evaluated with a short-range, screened interaction. If the singularity is properly taken into account, TD-EXX should indeed be similar to time-dependent Hartree-Fock. The results change then drastically, which can be understood by considering the effect on the polarizability $P_{1}\left(\mathbf{r}, \mathbf{r}^{\prime} ; \omega\right)$ : The Kohn-Sham eigenvalues obtained from the exact exchange potential are closer to the experimental band structures than LDA ones [138], but the selfenergy insertion in the first two terms in figure 9 increases the eigenvalue gap close to the Hartree-Fock result, leading to a far too high absorption threshold. In addition, the unscreened electron-hole interaction in the third term gives rise to a strongly overbound exciton with an incorrect line shape [132]. If the bare Coulomb potential is replaced by a screened interaction, then the self-energy insertion is comparable to the $G W$ approximation, which yields generally good quasiparticle band gaps (see e.g. [148]), and the exciton line shape is reproduced correctly by the screened electron-hole interaction $W$. (Such a screened kernel will be discussed insection 6). It has also been shown recently [149] that consistent inclusion of screening beyond EXX in the OEP potential reduces the Kohn-Sham eigenvalue gap and brings its value close to the LDA one; the discontinuity yields then the correct quasiparticle gap. A proper treatment of correlation (i.e. here: screening) is hence crucial for semiconductors, and computational schemes based only on exact exchange are not sufficient in this case. In addition, the linearisation of the equations as explained in section 5.1 is problematic for the gap-opening contribution $f(1)$, because the latter has to simulate a discontinuity [32]. This problem can be overcome by using the full, non-linearised term as explained in section 6 .

\section{Kernels from many-body perturbation theory}

In the previous section the Sham-Schlüter equation has been introduced. This equation makes a link between many-body perturbation theory (MBPT) and the density-functional framework. Since recent results indicate that exploiting this link can be very fruitful, the present section is dedicated to comparison and combination of the two approaches. In fact for the calculation of electronic excitations in solids there is a choice to be made. One can use Many-Body Perturbation Theory and solve the Bethe-Salpeter Equation, a precise but computationally demanding method, or use TDDFT, an in principle computationally more efficient approach, but with the limitations of the standard approximations for the exchangecorrelation potential and kernel (i.e. the TDLDA) outlined above. For an extended review comparing the two approaches, see e.g. [14].

The big advantage of TDDFT is that the polarizability is expressed as a variation of the density (where the latter is local in space and time); it can be directly obtained from the two-point equation (2.35). The BSE uses the more intuitive quasi-particle picture, which makes the task to identify efficient approximations easier. However, within MBPT one deals with four-point equations. In fact, a key quantity is the four-point reducible polarizability ${ }^{4} L$, that can be expressed in terms of the two-particle Green's function $G_{2}$ describing the propagation of two particles (for absorption, the relevant part describes the propagation of an electron and a hole):

$$
{ }^{4} L(1,2,3,4)={ }^{4} L_{0}(1,2,3,4)-G_{2}(1,2,3,4) .
$$

As in the previous section, in (6.1) the number "1" represents the set of position, time and spin variables $\left(\mathbf{r}_{1}, t_{1}, \sigma_{1}\right) . L_{0}$ is the disconnected part consisting of two one-particle Green's functions $G$ :

$$
{ }^{4} L_{0}(1,2,3,4)=\mathrm{i} G(1,3) G(4,2) .
$$


The function $L$ and its counterpart $\bar{L}$, defined in analogy to $\bar{\chi}$, satisfy a Dyson-like screening equation, known as the Bethe-Salpeter equation. When its kernel is approximated to first order in the screened Coulomb interaction $W$ it reads:

$$
\begin{aligned}
{ }^{4} \bar{L} & ={ }^{4} L_{0}+{ }^{4} L_{0}\left({ }^{4} \bar{v}-{ }^{4} W\right){ }^{4} \bar{L}, \\
{ }^{4} L & ={ }^{4} L_{0}+{ }^{4} L_{0}\left({ }^{4} v-{ }^{4} W\right){ }^{4} L
\end{aligned}
$$

In (6.3) we have defined the four-point extension of the Coulomb potential ${ }^{4} v(1,2,3,4)=$ $\delta(1,2) \delta(3,4) v(1,3)$, whereas ${ }^{4} W=\delta(1,3) \delta(2,4) W(1,2)$ is the four-point extension of the screened Coulomb potential. Note that the $\delta$-functions connect different indices in the two cases; this is due to the fact that the former stems from variations of the Hartree potential, whereas the latter is due to variations of an exchange-like self-energy contribution. Because of the way the indices are connected in this second case, the BSE can not be written in a two-point form. Instead, the measurable $\chi$ is obtained via a two-point contraction of ${ }^{4} L$, namely

$$
P^{\mathrm{red}}(1,2)=-L(1,1,2,2) .
$$

From the reduced polarizability $P^{\text {red }}$ that is understood to be time (or contour) -ordered, the causal response function $\chi$ can be inferred; the relation between the time-ordered and causal response is in fact $\chi(\omega>0)=P^{\text {red }}(\omega>0)$. Another useful quantity is the independent quasi-particle polarizability, $P_{0}(1,2)=-L_{0}(1,1,2,2)$. Note that this is not equal to $\chi_{\mathrm{KS}}=-\mathrm{i} G_{\mathrm{KS}} G_{\mathrm{KS}}$. The expression looks similar, but the Kohn-Sham states and eigenvalues in $\chi_{\mathrm{KS}}$ are now replaced by their MBPT counterparts, which are, on this level of approximation, usually determined in the quasi-particle GW approximation [129] (to be precise, most realistic calculations use GW eigenvalues and Kohn-Sham wavefunctions to build $G$ ).

As the two sets of equations (2.35), (3.7) and (6.3) have a similar mathematical structure, it is natural to try to extract information about the TDDFT exchange-correlation kernel through their comparison. Different authors reached very similar expressions for the exchange-correlation kernel starting from the BSE but using different approaches $[29,30,31,32,150,146,33,35]$. Tested for real materials, these kernels proved to be successful in reproducing the quality of the BSE spectra via a TDDFT formalism. In present implementations their computational cost still remains comparable to the cost of solving the BSE but memory requirements are significantly reduced, and the expressions can be rewritten leading to algorithms with better scaling. Moreover (see section 8), they are a very convenient starting point for further approximations. In the following we summarise some of these derivations (section 6.2). We will also briefly outline how the combination BSETDDFT can be used to improve upon current approximations of MBPT, by introducing the TDDFT concept into MBPT (section 6.3). Some applications are shown in section 6.4.

\subsection{The exchange-correlation kernel from the Sham-Schlüter equation}

Let us first come back to the non-linearised Sham-Schlüter equation (5.1). As pointed out in section 5 , it is equivalent to the condition $n(\mathbf{r}, t)=-i G\left(\mathbf{r}, \mathbf{r}^{\prime}, t, t^{+}\right)$. In order to obtain an exact expression for $f_{\mathrm{xc}}$, Bruneval et al. [150] started from this equality and took its functional derivative with respect to the density. $\delta G / \delta n=-G\left(\delta G^{-1} / \delta n\right) G$ leads to

$$
\mathrm{i} G(13) G\left(41^{+}\right) \frac{\delta G^{-1}(34)}{\delta n(2)}=\delta(12) .
$$


Since the same exact density, and hence the same Hartree potential, should also be obtained from the Kohn-Sham potential $v_{\mathrm{KS}}=V+v_{\mathrm{xc}}$ one can write

$$
G^{-1}(12)=G_{0}^{-1}(12)-\delta(12)\left(v_{\mathrm{KS}}(1)-v_{\mathrm{xc}}(1)\right)-\Sigma(12) .
$$

As $\delta G_{0}^{-1} / \delta n=0,(6.5)$ becomes

$$
P_{0}(13) \chi_{\mathrm{KS}}^{-1}(32)-\mathrm{i} G(13) G\left(41^{+}\right) \frac{\delta \Sigma(34)}{\delta n(2)}-P_{0}(13) f_{\mathrm{xc}}(32)=\delta(12) .
$$

As in the linearised case section 5, the exact $f_{\mathrm{xc}}$ turns out to consist of two terms $f_{\mathrm{xc}}=f_{\mathrm{xc}}^{(1)}+f_{\mathrm{xc}}^{(2)}[33,150]$. Those read now

$$
f_{\mathrm{xc}}^{(1)}(12)=\chi_{\mathrm{KS}}^{-1}(12)-P_{0}^{-1}(12)
$$

and

$$
f_{\mathrm{xc}}^{(2)}(12)=-\mathrm{i} P_{0}^{-1}\left(1,1^{\prime}\right) G\left(1^{\prime} 3\right) G\left(41^{\prime+}\right) \frac{\delta \Sigma(34)}{\delta n(2)}
$$

$f_{\mathrm{xc}}^{(1)}$ exactly changes the Kohn-Sham response function into the independent QP one, in particular, it solves hence the band gap problem. $f_{\mathrm{xc}}^{(2)}$ accounts for the electron-hole interaction.

Altogether, TDDFT yields then for the irreducible polarizability

$$
\chi=\chi_{\mathrm{KS}}+\chi_{\mathrm{KS}}\left(v+\chi_{\mathrm{KS}}^{-1}-P_{0}^{-1}+f_{\mathrm{xc}}^{(2)}\right) \chi=P_{0}+P_{0}\left(v+f_{\mathrm{xc}}^{(2)}\right) \chi .
$$

To get an explicit approximation for $f_{\mathrm{xc}}$, one has to choose a starting approximation for the self-energy and the Green's functions. A simple choice could be to take $\Sigma, G$ and $P_{0}$ as derived from a local and adiabatic exchange-correlation potential, e.g. the LDA one. This leads of course to the TDLDA and the GWT approach of Ref. [151]. A better choice is to start from the GW approximation for $\Sigma$, taking $W$ as a screened (e.g. static RPA) Coulomb interaction. For the functional derivative, it is then reasonable (i) to neglect the derivative of $W$ as usually done in the BSE: (ii) to approximate $\delta G / \delta n=-G\left(\delta G^{-1} / \delta n\right) G$ by $G P_{0}^{-1} G$. The latter approximation truncates the chain of derivatives $\delta \Sigma / \delta n$ that would appear if one continued to calculate all terms of $\delta G^{-1} / \delta n$ (Note that this is equivalent to supposing $G$ be created by a local potential). Equation (6.9) yields then

$$
f_{\mathrm{xc}}^{(2)}(34)=\int d 5 d 6 d 7 P_{0}^{-1}(36) G(65) G\left(5^{\prime} 6\right) W\left(55^{\prime}\right) G(57) G\left(75^{\prime}\right) P_{0}^{-1}(74) .
$$

Equation (6.11) is the successful electron-hole exchange-correlation kernel of Refs. [29, 30, $31,32,150,146,33,150,35]$. Comparison with (5.4) shows that the two expressions are very similar; however, now the formerly bare interaction is screened, which removes the main problems of the bare exchange approach. Moreover, QP instead of Kohn-Sham Green's functions appear throughout. This reflects the fact that (6.8) has replaced (5.5), which is important since, as pointed out above, the linearisation of this contribution is in fact problematic.

\subsection{Comparison of TDDFT and MBPT}

It is instructive to have a look also at the alternative derivations of this kernel. In fact, instead of starting from the density it may be more straightforward to start from the observation 
that MBPT and TDDFT should yield the same two-point response function. Since $\chi$ can in principle be obtained via $P^{\text {red }}$ from (6.4), one could invert the screening equation and determine $f_{\mathrm{xc}}$ from $f_{\mathrm{xc}}=\chi_{\mathrm{KS}}^{-1}-\chi^{-1}-v[152,153]$. The latter relation is of course not of practical interest, but can give insight about general features of the kernel, like its overall frequency behaviour, an idea that has been exploited in [154].

Reining et al $[29,30]$ instead used the equality of the response functions in order to obtain an approximate expression for the exchange-correlation kernel of TDDFT by mapping directly the matrix elements of the BSE (6.3) onto the matrix elements of the TDDFT linear response equation (2.35). The derivation starts by rewriting the TDDFT Dyson equation (2.35) in a four-point formalism

$$
{ }^{4} \bar{\chi}={ }^{4} \chi_{\mathrm{KS}}+{ }^{4} \chi_{\mathrm{KS}}\left({ }^{4} \bar{v}+{ }^{4} f_{\mathrm{xc}}\right){ }^{4} \bar{\chi},
$$

where the four-point Coulomb interaction ${ }^{4} \bar{v}(1,2,3,4)=\delta(1,2) \delta(3,4) \bar{v}(1,3)$ and the fourpoint kernel ${ }^{4} f_{\mathrm{xc}}(1,2,3,4)=\delta(1,2) \delta(3,4) f_{\mathrm{xc}}(1,3)$ are defined [30].

On the other hand, the BSE has the same structure as (6.12) (cf. (6.3a)), but with ${ }^{4} \chi_{\mathrm{KS}}$ replaced by its four-point MBPT counterpart ${ }^{4} L_{0}$, and the TDDFT kernel replaced by

$$
{ }^{4} K=\delta(1,2) \delta(3,4) \bar{v}(1,3)-\delta(1,3) \delta(2,4) W(1,2) .
$$

In $[29,30]$, an approximation for both $f_{\mathrm{xc}}^{(1)}$ and $f_{\mathrm{xc}}^{(2)}$ were derived; since in practise it is convenient to use the exact expression (6.8) for the former contribution, only the derivation of $f_{\mathrm{xc}}^{(2)}$ will be discussed in the following.

We thus replace $\chi_{\mathrm{KS}}$ by $P_{0}$ and focus on the second part of the exchange-correlation kernel. The first term of the total kernel in both TDDFT and BSE, i.e., the bare Coulomb interaction $\bar{v}$, is identical. One might hence try to identify the remaining part of the kernel $f_{\mathrm{xc}}^{(2)}$ with the screened Coulomb interaction of the BSE kernel (6.13):

$$
\delta(1,2) \delta(3,4) f_{\mathrm{xc}}^{(2)}(1,3) \leftrightarrow-\delta(1,3) \delta(2,4) W(1,2)
$$

However, as the $\delta$ - functions do not contract the same indices expression (6.14) cannot be an equality. Different approaches have been proposed to overcome this difficulty and nevertheless use the similarity of the equations:

i) the assumption that kernels could be similar for a limited number of transitions:

For this approach, it is useful to write (6.14) on a basis of pairs of Kohn-Sham LDA states (transition space) [155]

$$
F_{(v c \mathbf{k})\left(v^{\prime} c^{\prime} \mathbf{k}^{\prime}\right)}^{\mathrm{TDDFT}} \leftrightarrow F_{(v c \mathbf{k})\left(v^{\prime} c^{\prime} \mathbf{k}^{\prime}\right)}^{\mathrm{BSE}},
$$

with the matrix elements

$$
\begin{aligned}
& F_{(v c \mathbf{k})\left(v^{\prime} c^{\prime} \mathbf{k}^{\prime}\right)}^{\mathrm{TDDFT}}=2 \int d^{3} r d^{3} r^{\prime} \Phi(v \mathbf{k} c \mathbf{k}, \mathbf{r}) f_{\mathrm{xc}}^{(2)}\left(\mathbf{r}, \mathbf{r}^{\prime}, \omega\right) \Phi^{*}\left(v^{\prime} \mathbf{k}^{\prime} c^{\prime} \mathbf{k}^{\prime}, \mathbf{r}^{\prime}\right) \\
& F_{(v c \mathbf{k})\left(v^{\prime} c^{\prime} \mathbf{k}^{\prime}\right)}^{\mathrm{BSE}}=-\int d^{3} r d^{3} r^{\prime} \Phi\left(v \mathbf{k} v^{\prime} \mathbf{k}^{\prime}, \mathbf{r}\right) W\left(\mathbf{r}, \mathbf{r}^{\prime}, 0\right) \Phi^{*}\left(c \mathbf{k} c^{\prime} \mathbf{k}^{\prime}, \mathbf{r}^{\prime}\right),
\end{aligned}
$$

where $\Phi\left(i \mathbf{k} j \mathbf{k}^{\prime}, \mathbf{r}\right)$ is the product of a pair of Kohn-Sham wavefunctions; The indices $\{i, \mathbf{k}\}$ stand for the band-index and momentum of the Kohn-Sham state. Only the resonant contribution is shown (transitions from occupied to empty states) and the momentum transfer is supposed to be vanishing since the approach has been derived for optical spectra; 
both conditions can however easily be generalised. As usual in calculations based on the Bethe-Salpeter equation, $W$ is moreover supposed to be static.

Note that (i) In practise, the equality in (6.15) is then imposed for transitions belonging to a certain frequency range. The TDDFT spectra will match the BSE spectra only in this region. (ii) $F_{(v c \mathbf{k})\left(v^{\prime} c^{\prime} \mathbf{k}^{\prime}\right)}^{\mathrm{TDDT}}$ is now a static quantity over that energy range, as a consequence of the static approximation for $W$. (However, since because of (i) a different kernel will be obtained for a different group of transitions, there is an overall effective frequency dependence).

Reining et al. [29] have formally inverted (6.15). A Fourier transform to reciprocal space leads then to

$$
f_{\mathrm{xc}}^{(2)}\left(\mathbf{q} \rightarrow 0, \mathbf{G}, \mathbf{G}^{\prime}\right)=\sum_{v c \mathbf{k} v^{\prime} c^{\prime} \mathbf{k}^{\prime}} \Phi_{\mathbf{G}}^{-1}(v \mathbf{k} c \mathbf{k} ; \mathbf{q} \rightarrow 0) F_{(v c \mathbf{k})\left(v^{\prime} c^{\prime} \mathbf{k}^{\prime}\right)}^{\mathrm{BSE}}\left(\Phi^{*}\right)_{\mathbf{G}^{\prime}}^{-1}\left(v^{\prime} \mathbf{k}^{\prime} c^{\prime} \mathbf{k}^{\prime} ; \mathbf{q} \rightarrow 0\right) .
$$

The TDDFT exchange-correlation kernel derived in this way has correct asymptotic behaviour (see the discussion in section 8); it stems from the asymptotic behaviour of the $\Phi$, not from the Coulomb interaction $W$ as one might have suspected. The inversion of the matrices $\Phi$ is purely formal; the kernel (6.17) itself was in fact not evaluated in Ref. [29], but used to derive a TDDFT-like screening equation. A more direct way to arrive at the same screening equation along similar lines has been proposed by Sottile et al. [30]. One starts by writing the TDDFT-like response equation (3.7) in a symmetrised form (the same can be done with (2.35)):

$$
\bar{\chi}=P_{0}\left(P_{0}-P_{0} \bar{v} P_{0}-P_{0} f_{\mathrm{xc}}^{(2)} P_{0}\right)^{-1} P_{0} .
$$

It appears that in order to calculate $\bar{\chi}$ one only needs $P_{0} f_{\mathrm{xc}}^{(2)} P_{0}$, and not $f_{\mathrm{xc}}^{(2)}$ explicitly. This integral contains sums over transitions involving $F_{(v c \mathbf{k})\left(v^{\prime} c^{\prime} \mathbf{k}^{\prime}\right)}^{\mathrm{TDDFT}}$. Enforcing hence the equality in (6.15) yields

$$
P_{0} f_{\mathrm{xc}}^{(2)} P_{0}=T_{2}
$$

where $T_{2}$ is calculated as :

$$
T_{2}\left(\mathbf{G}, \mathbf{G}^{\prime}, \omega\right)=\frac{2}{N_{k}^{2}} \sum_{v c \mathbf{k} v^{\prime} c^{\prime} \mathbf{k}^{\prime}} \frac{\Phi^{*}(v \mathbf{k} c \mathbf{k} ; \mathbf{G})}{\varepsilon_{c \mathbf{k}}^{\mathrm{QP}}-\varepsilon_{v \mathbf{k}}^{\mathrm{QP}}-\omega} F_{(v c \mathbf{k})\left(v^{\prime} c^{\prime} \mathbf{k}^{\prime}\right)}^{\mathrm{BSE}} \frac{\Phi\left(v^{\prime} \mathbf{k}^{\prime} c^{\prime} \mathbf{k}^{\prime} ; \mathbf{G}^{\prime}\right)}{\varepsilon_{c^{\prime} \mathbf{k}^{\prime}}^{\mathrm{QP}}-\varepsilon_{v^{\prime} \mathbf{k}^{\prime}}^{\mathrm{QP}}-\omega} ;
$$

(here we give again only the expression for the resonant contribution, which is the dominant term in absorption spectra).

This is a result that can be used in practise. The first calculation has been done for bulk silicon; the result shown in figure 11 obtained by Sottile et al. shows almost perfect agreement with a result obtained by solving the BSE.

From a computational point of view, one still has to calculate $W$; in today's implementation moreover the two-particle matrix elements $W_{(v c \mathbf{k})\left(v^{\prime} c^{\prime} \mathbf{k}^{\prime}\right)}$ are calculated, which is often the most expensive part of a BSE calculation. In principle the sum over transitions could however be performed in a different order and the latter calculation avoided. Moreover by studying (6.20), one can derive model kernels, which can decrease drastically the computational cost. The performance of the kernel as well as efficient approximations will be illustrated in section 8 .

ii) A perturbative approach leading to the same exchange-correlation kernel was proposed by Adragna et al . [31]: 


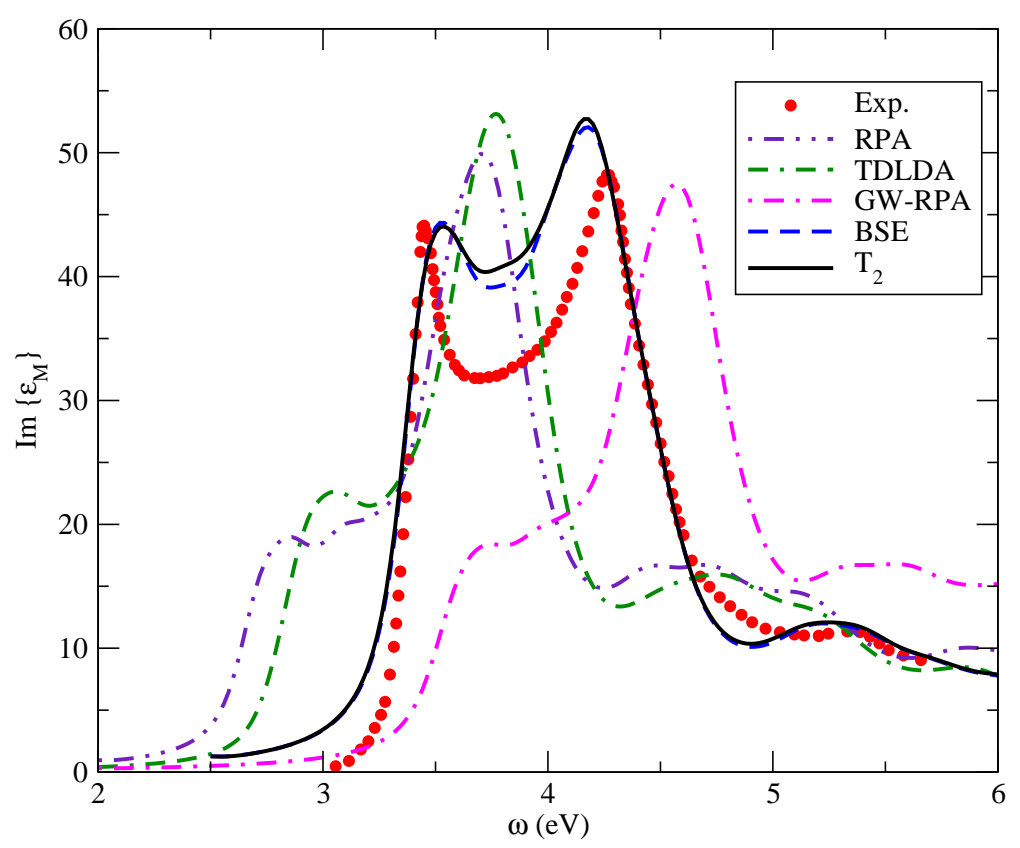

Figure 11. Imaginary part of the macroscopic dielectric function for Si [30]. Dots: experiment [93]; dash-dot-dot line: RPA calculation; dash-dash-dot line: TDLDA calculation; dash-dot line: GW-RPA calculation; dash line: BSE calculation; solid line: $\mathrm{T}_{2}$ kernel.

Their starting point is again the requirement that the TDDFT and the BSE two-point response function should be equal. The polarizabilities are then developed perturbatively to the same order. Truncation to first order leads again to (6.19) with (6.20). Marini et al. used then this kernel for the calculation of optical absorption of insulators, including a bound exciton, and loss spectra at non-vanishing momentum transfer[32]. Both cases were in good agreement with experiments. It was also shown that the inclusion of the second-order term in the perturbative expansion did not affect significantly the calculated spectra. For more details see chapters 10 and 20 of Ref.[38]

iii) The perturbative approach was also explored in terms of Feynman diagrams $[146,156,33,101]$ :

Stubner, Tokatly and Pankratov [33] derived an integral equation which leads to (6.20) when non-locality beyond the first order is neglected. Also the separation of $f_{\mathrm{xc}}^{(1)}$ and $f_{\mathrm{xc}}^{(2)}$ naturally comes out from that derivation.

Finally we mention the work of von Barth et al. [35]: here the kernel is derived from a functional approach. Again, this yields $f_{\mathrm{xc}}^{(1)}$ in its linearised version as well as the above approximation for $f_{\mathrm{xc}}^{(2)}$, when $G W$ diagrams are chosen. The advantage of deriving a kernel as double derivative of a functional is that the symmetry of the expression guarantees that important conservation laws are fulfilled. This kernel is hence the result of the work of several groups, the great majority (although not all) of them being members of a network called "nanoquanta". For convenience we therefore suggest to call it the "nanoquanta kernel". 


\subsection{Combining TDDFT and MBPT}

In order to complete the section about links between MBPT and TDDFT, it is useful to remind that improved TDDFT response functions and kernels can in turn be inserted into many-body calculations in order to go beyond existing approximations, in particular the RPA-based GW approximation.

The kernel of linear-response TDDFT stems from the variations of the Hartree and exchange-correlation potentials with respect to the density. Within MBPT, the variations are taken with respect to the one-particle Green's function. However, when one is only interested in the self-energy and the Green's function (and not in the full four-point response function) also in the case of MBPT these variations can be created by a local external potential, for which the Runge-Gross theorem holds [16]. As a consequence, also in the MBPT scheme it is possible to rely on the fact that the density variations determine the physics of excitations.

Starting from this observation, Bruneval et al suggested an alternative way to find approximations for the self-energy $\Sigma$ [150]. This operator, that accounts for all the manybody effects on the one-particle Green's function beyond the Hartree term and is a key quantity of MBPT, is defined as [129, 130]

$$
\Sigma(1,2)=\mathrm{i} \int d 3 d 4 d 5 G(1,4) \Gamma(4,2 ; 5) \frac{\delta V(5)}{\delta U(3)} v\left(3,1^{+}\right)
$$

where $G$ is the one-particle Green's function, $U$ is a local external potential, $V(1)=$ $U(1)+v_{\mathrm{H}}(1)$ is the total classical potential - including also the Hartree contribution $v_{\mathrm{H}}$, and $\delta V(5) / \delta U(3)=\epsilon^{-1}(5,3)$. The irreducible vertex function $\Gamma$ is defined as

$$
\Gamma(1,2,3)=-\frac{\delta G^{-1}(1,2)}{\delta V(3)}=\delta(1,3) \delta(2,3)+\frac{\delta \Sigma(1,2)}{\delta V(3)}
$$

Bruneval et al proposed to replace the chain rule usually employed to obtain $\delta \Sigma / \delta V$, namely $(\delta \Sigma / \delta G)(\delta G / \delta V)[157,130]$, with the alternative chain rule $(\delta \Sigma / \delta \rho)(\delta \rho / \delta V)$. This step is justified by the one-to-one relation between the time-dependent density and external potential, or consequently between the density and the classical potential $V$. Equation (6.22) then becomes

$$
\Gamma(1,2 ; 3)=\delta(1,3) \delta(2,3)+\int d 4 \frac{\delta \Sigma(1,2)}{\delta \rho(4)} P(4,3)
$$

where $P=\delta \rho / \delta V$ is the irreducible polarizability

$$
P(1,2)=-i \int d 3 d 4 G(1,3) G(4,1) \Gamma(3,4,2) .
$$

On the other hand, if one multiplies (6.23) with two Green's functions $G$ and integrates, the result is

$$
P(1,2)=P_{0}(1,2)+\int d 3 d 4 P_{0}(1,3) f_{\mathrm{xc}}^{(2)}(3,4) P(4,2)
$$

with (6.9).

This constitutes hence an alternative derivation of the exact kernel. Moreover, once $P$ is known (6.23) allows one to calculate the three-point vertex, and hence an improved selfenergy, without solving a four-point integral equation. It has been shown [150] that, besides 


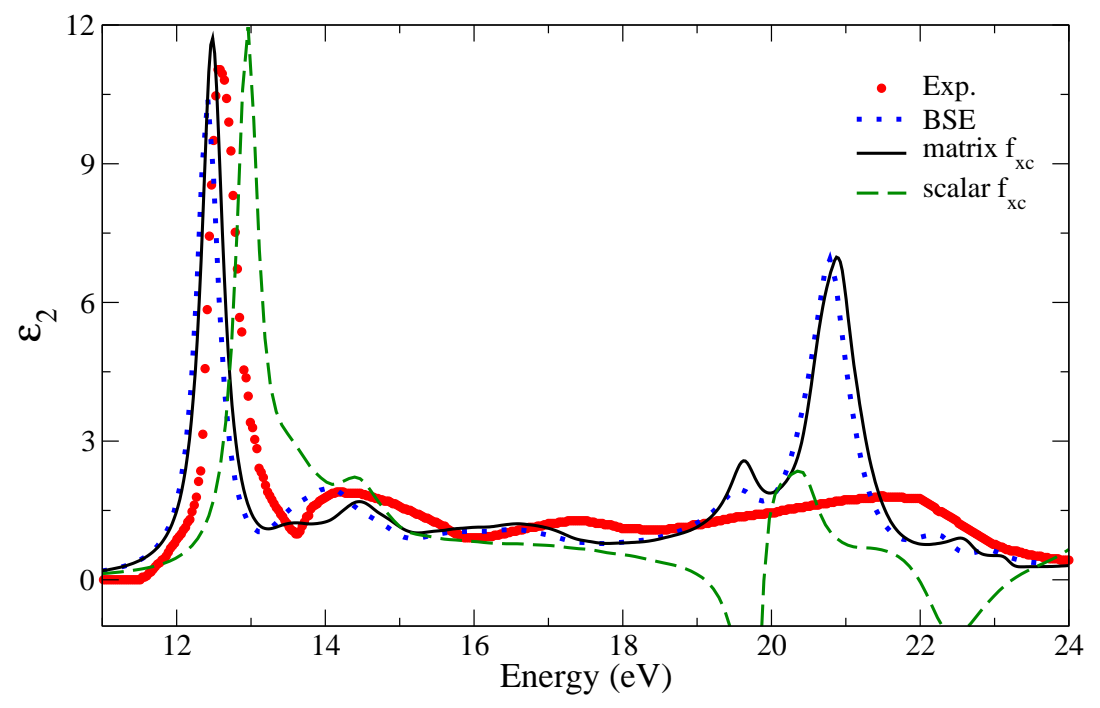

Figure 12. Optical absorption spectra calculated within the BSE (dots), and TDDFT (solid line) compared with experiments (circles) [88]. The results obtained using a scalar $f_{\mathrm{Xc}}$ (dashed line) are shown to stress the importance of the $\mathbf{G}, \mathbf{G}^{\prime} \neq 0$ terms in $f_{\mathrm{xc}}$.

the better description of the test-charge-test-charge screened $W$, a major contribution to the correction beyond GW stems from the induced exchange-correlation potential that acts on an additional particle or hole on top of the induced Hartree potential; only the latter is contained in GW (moreover, it is approximated in RPA).

\subsection{Applications}

When coming to practical calculations it should be noted that in general (i) as mentioned above, only $f_{\mathrm{xc}}^{(2)}$ is explicitly used, whereas the GW correction is included by using $P_{0}$; (ii) most calculations are restricted to the resonant contribution; (iii) the diagonal contribution $F_{(v c \mathbf{k})(v c \mathbf{k})}^{\mathrm{BSE}}$ is extracted from $f_{\mathrm{xc}}^{(2)}$ and included in $P_{0}$. Strictly speaking, this contribution should be zero in a solid, but for a finite k-point sampling it is non-vanishing. These three points are important for the quality of the final results.

An example of the application of the BSE-derived kernel (6.20) to real systems can be found in figure 12 [32], where the optical absorption spectrum of $\mathrm{LiF}$ is shown. One can observe that, as anticipated, the agreement between the BSE calculation and the TDDFT calculation using the BSE-derived kernel is excellent. The importance of the matrix character of $f_{\mathrm{xc}}$ (i.e. the importance of $\mathbf{G}, \mathbf{G}^{\prime} \neq 0$ terms) in wide-gap insulators is demonstrated by looking at the curve obtained with a scalar $\left(\mathbf{G}=\mathbf{G}^{\prime}=0\right) f_{\mathrm{xc}}$. Although the kernel is strongly frequency dependent, only the main peak is reproduced correctly, while some unphysical regions of negative absorption appear at higher energies. In contrast, when dealing with systems with continuum exciton effects ( $\mathrm{Si}$, diamond, etc.) the use of the head of $f_{\mathrm{xc}}$ is often sufficient to recover the BSE spectrum. This is related to the different degree of inhomogeneity of the induced density in simple semiconductors and wide-gap insulators.

The BSE-derived kernel (6.20) has also been successfully applied to the study of lowdimensional systems. An example are conjugated polymers. We have already pointed out in section 3.2 that simple adiabatic local and gradient corrected functionals fail in describing 


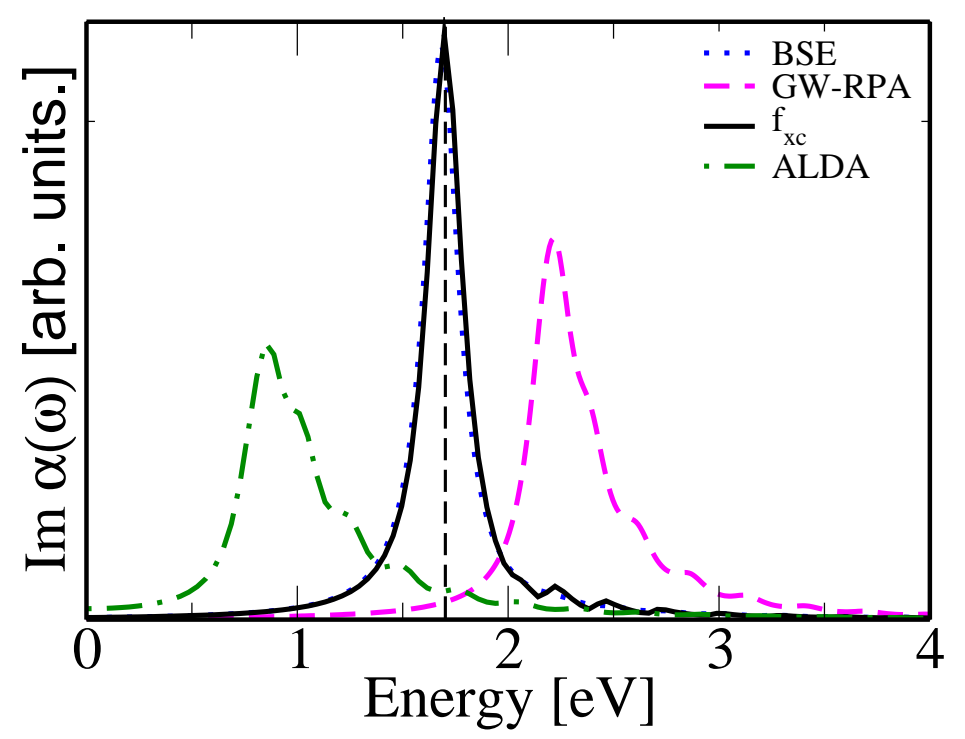

Figure 13. Optical absorption spectra of polyacethylene compared with experiment (vertical dashed-line) [160, 161]. The BSE calculation (dots) and the TDDFT calculation using the many-body $f_{\mathrm{xc}}$ (solid line) are in excellent agreement with the experiment. GW-RPA (dashed-line) and TDLDA calculations (dot-dashed line) are also shown.

the dielectric response of long molecular chains [24]. Current density functional approaches can partially solve this problem $[158,159]$. The non-local frequency dependent BSE-derived kernel restores the good agreement between the calculated and the measured polarizabilities. This can be observed in figure 13, where the calculated optical absorption spectrum of polyacethylene is compared with experimental data [160, 161].

In brief, the BSE derived $f_{\mathrm{xc}}$ kernel is able to reproduce the optical and energy-loss spectra of a large class of materials including semiconductors and large band-gap insulators $[30,32]$, as well as systems of low dimensionality [160]. Although calculations are today still relatively cumbersome, improved algorithms and/or efficient approximations are expected to make this approach clearly competitive with respect to a BSE calculation, when one is interested in an efficient determination of spectra.

\section{Current-density functionals}

Finally, we would like to briefly outline an alternative way to approach the question of going beyond the ALDA. In fact, many problems of the latter, including its dramatic failure for optical absorption spectra of insulators, are related to the fact that it is derived only from the local density. As a consequence, the effective potential inside a bulk solid is insensitive to the accumulation of surface charges, which may have a large influence on the measured material properties in actual experiments. In particular, they give rise to a long range exchange-correlation potential that counteracts an applied external field, thus reducing the observable polarizability. As the density inside the bulk does not change in this case, such a counteracting term cannot be reproduced by a purely local approximation. Instead, the exchange-correlation potential must depend in a non-local manner on the global density distribution. This is difficult to achieve with explicit density functionals, which are, like the 
ALDA, typically derived from the homogeneous electron gas. Implicit density functionals constructed from the Kohn-Sham wave functions do not suffer from the same limitation, but the large computational cost makes it difficult to incorporate high-order correlation terms in practise. An alternative approach to include non-locality is given by time-dependent currentdensity-functional theory, because, in contrast to the density $n(\mathbf{r}, t)$, the current density $\mathbf{j}(\mathbf{r}, t)$ can be used as a local indicator of global changes in the system. For example, its behaviour correctly reflects the charge transport through the bulk between two solid surfaces upon polarisation [162].

Current-density-functional theory was originally developed as a generalisation of TDDFT for time-dependent magnetic fields $[163,56]$. In this case, in addition to the scalar potential $v_{\text {ext }}(\mathbf{r}, t)$, the Hamiltonian

$$
\begin{aligned}
\hat{H}(t)=\sum_{\sigma} \int \hat{\psi}_{\sigma}^{\dagger}(\mathbf{r})\left(\frac{1}{2}[-\mathrm{i} \nabla\right. & \left.\left.+\mathbf{A}_{\text {ext }}(\mathbf{r}, t)\right]^{2}+v_{\text {ext }}(\mathbf{r}, t)\right) \hat{\psi}_{\sigma}(\mathbf{r}) \mathrm{d}^{3} r \\
& +\frac{1}{2} \sum_{\sigma, \sigma^{\prime}} \int \hat{\psi}_{\sigma}^{\dagger}(\mathbf{r}) \hat{\psi}_{\sigma^{\prime}}^{\dagger}\left(\mathbf{r}^{\prime}\right) v\left(\mathbf{r}-\mathbf{r}^{\prime}\right) \hat{\psi}_{\sigma^{\prime}}\left(\mathbf{r}^{\prime}\right) \hat{\psi}_{\sigma}(\mathbf{r}) \mathrm{d}^{3} r \mathrm{~d}^{3} r^{\prime}
\end{aligned}
$$

contains an external vector potential $\mathbf{A}_{\text {ext }}(\mathbf{r}, t)$. The conjugate variable that couples to the latter is the current density, defined in analogy to (2.5) but with $-\mathrm{i} \nabla+\mathbf{A}_{\text {ext }}(\mathbf{r}, t)$ instead of the canonical momentum operator $-i \nabla$. Within this framework the auxiliary Kohn-Sham system is chosen in such a way that it yields not only the same density but also the same current density as the original interacting electron system. As a necessary prerequisite to achieve non-interacting $v$-representability of both quantities, the effective vector potential $\mathbf{A}_{\text {eff }}(\mathbf{r}, t)=\mathbf{A}_{\text {ext }}(\mathbf{r}, t)+\mathbf{A}_{\mathrm{xc}}(\mathbf{r}, t)$ in the Kohn-Sham equations must then feature a non-vanishing exchange-correlation contribution, because changes in the scalar potential alone have no effect on the transverse component of the current [164]. The scalar potential can actually be eliminated at all times through the gauge transformation $\mathbf{A}_{\text {ext }}(\mathbf{r}, t) \rightarrow \mathbf{A}_{\text {ext }}(\mathbf{r}, t)+\nabla \Lambda(\mathbf{r}, t)$ and $V_{\text {ext }}(\mathbf{r}, t) \rightarrow v_{\text {ext }}(\mathbf{r}, t)-\partial \Lambda(\mathbf{r}, t) / \partial t$, if the gauge function satisfies $\partial \Lambda(\mathbf{r}, t) / \partial t=v_{\text {ext }}(\mathbf{r}, t)$ with the initial condition $\Lambda\left(\mathbf{r}, t_{0}\right)=0$. Besides, the evolution of the density is completely determined through the static equilibrium value $n\left(\mathbf{r}, t_{0}\right)=n_{\mathrm{GS}}(\mathbf{r})$ and the continuity equation (2.8). Therefore, current-density-functional theory can be formulated solely in terms of vector potentials and with the current density as the only independent variable. An elegant proof of their one-to-one correspondence, which includes the Runge-Gross theorem as a special case, was given by Vignale [165]; the vector potential is determined uniquely up to a gauge transformation that does not alter the initial state.

For practical purposes the exchange-correlation vector potential must be specified as a functional of the current density. Fortunately, it turned out that a consistent gradient expansion around the limit of the homogeneous electron gas is possible in this approach. By investigating weakly inhomogeneous systems, Vignale and Kohn [166] thus derived an approximation for the induced field that includes dynamic exchange and correlation effects and depends only on the local current density. The resulting expression can be interpreted in terms of viscoelastic stresses in the electron fluid [167, 168]. So far the approximation of Vignale and Kohn has mainly been applied to plasmon line widths in semiconductor quantum wells within the one-band effective-mass model [169, 170, 171] as well as to one-dimensional polymers and other extended macromolecules, where it leads to significant improvements in the polarizabilities over a treatment within the ALDA $[172,159]$. A simplified version has also been used in the context of solid-state physics to calculate optical absorption spectra [158]. 
The results reproduce the essential spectral features for several common semiconductors and insulators, including the excitonic $E_{1}$ resonance in silicon, and are in much better agreement with experimental data than the ALDA. However, the absorption threshold is too low, and it remains unclear whether the residual discrepancies are due to the VignaleKohn approximation itself or to the additional simplifications of the implementation. It should be noted that the simplified formula applied in Ref. [158] is very similar to the long range approximation $[29,34]$ that will be discussed in the next section. This explains both its success in describing the line shape and its insuccess in opening the band gap without the help of an explicit energy shift (a task that is carried out by the GW corrections in the case of the long range kernel of section 8).

\section{Simple models}

In section 6 we introduced a class of parameter-free kernels that are particularly successful for the description of optical absorption in solids - the kernels derived from the BSE. Even though they have a potentially reduced computational effort with respect to the BSE, calculations using these kernels are however still significantly more involved than those within the RPA or the TDLDA. Therefore, the question of finding simple and efficient, but also reliable, models for $f_{\mathrm{xc}}$ is still open. In this quest, there are several important lessons to be learnt from the BSE-derived kernels. In the following we discuss some model kernels, inspired by the BSE, that combine simplicity with a reasonably good description of response properties.

\subsection{Long-range exchange-correlation kernels}

One of the most striking characteristic of the TDLDA kernel is that it is static and local in space. Therefore, one can expect that the inclusion of either dynamical (memory) effects, long-range nonlocal terms $[21,146]$ or both improves, in principle, the results yielded by the simple TDLDA. In this section, we introduce some model kernels, obtained by approximating the BSE-derived kernel of (6.9) and (6.19), that accounts for such further terms.

The exchange-correlation kernel (6.9),(6.19) contains a long-range contribution (LRC) of the form $1 / q^{2}$. This LRC is instead completely absent within the TDLDA, as the TDLDA kernel goes to a constant in the limit $q \rightarrow 0$. The simplest model that exhibits the correct LRC has the form

$$
f_{\mathrm{xc}}^{\text {static }}(\mathbf{q})=-\frac{\alpha^{\text {static }}}{q^{2}},
$$

where $\alpha^{\text {static }}$ is a material dependent parameter. The use of this particular form can be motivated starting from the BSE, following the lines of section 6 [29]. In fact, the function $\Phi_{\mathbf{G}=0}(v \mathbf{k} c \mathbf{k} ; \mathbf{q})$ in $(6.17)$ goes to zero as $\Phi \sim q$ for $q \rightarrow 0$. Since in this limit $F_{(v c \mathbf{k})\left(v^{\prime} c^{\prime} \mathbf{k}^{\prime}\right)}^{\mathrm{BSE}}$ behaves like a constant, this implies that $f_{\mathrm{xc}}\left(\mathbf{q}, \mathbf{G}=0, \mathbf{G}^{\prime}=0\right)$ goes as $1 / q^{2}$ in the optical limit. Moreover, as it can be seen in figure 14 the LRC to the exchange-correlation kernel is inversely proportional to the macroscopic dielectric constant $\epsilon_{\infty}$ [34].

Note that there is also a positive long-range contribution to the exchange-correlation kernel stemming from the QP shift of eigenvalues (as predicted in Ref. [173]), that competes with the negative one resulting from the BSE, i.e. the electron-hole interaction. This contribution is contained in (6.8). In references $[29,34]$ it was shown that the LRC alone is sufficient to reproduce the contribution of the electron-hole interaction $f_{\mathrm{xc}}^{(2)}$ to the optical spectra of simple semiconductors. The same is not true concerning the the self-energy 


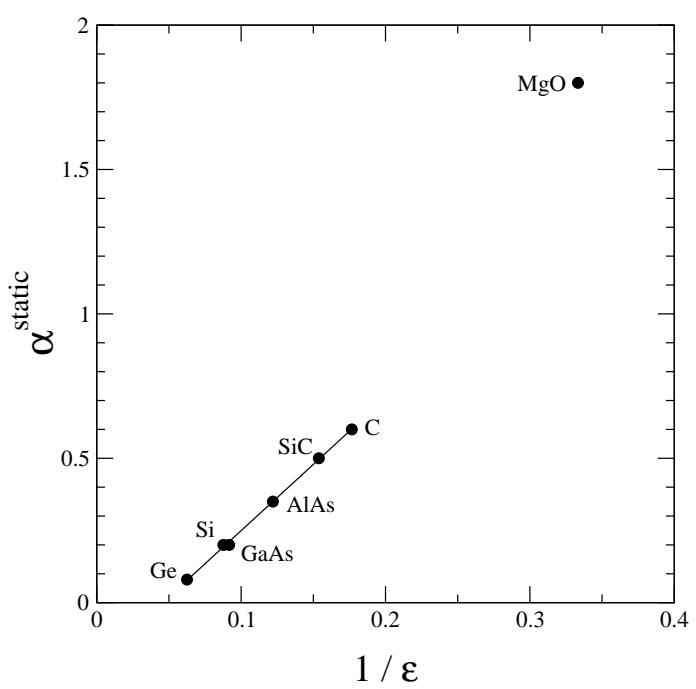

Figure 14. Material dependence of $\alpha^{\text {static }}$ with respect to the inverse of the dielectric constant [34].

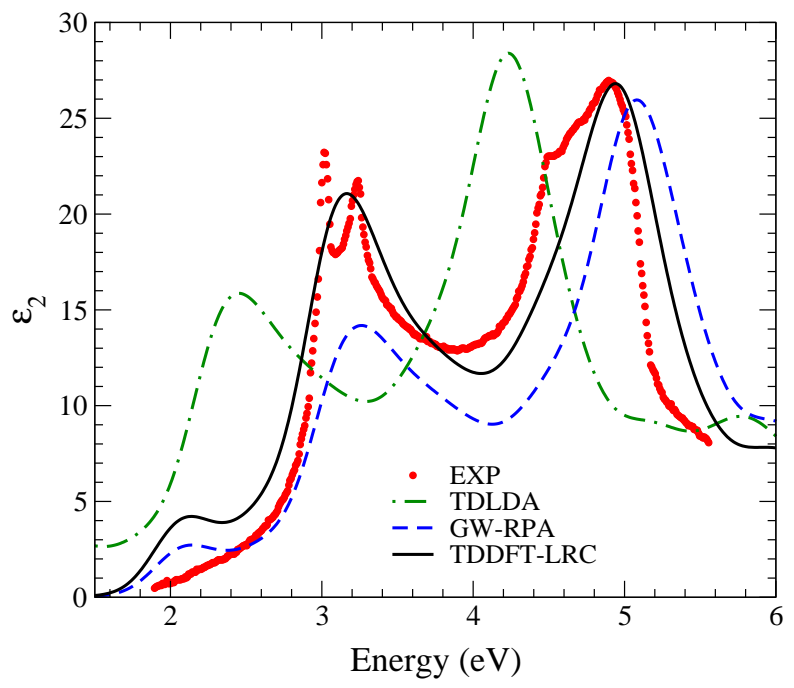

Figure 15. Imaginary part of the macroscopic dielectric function of GaAs [34]. Dots: experiment [174]; dot-dashed curve: TDLDA; dashed curve: GW-RPA; solid line: TDDFT-LRC.

contribution $f_{\mathrm{xc}}^{(1)}$, where a LRC approximation does not work. For this reason in the following discussion we will focus on models for the term $f_{\mathrm{xc}}^{(2)}$.

Figure 15 shows the optical absorption spectra of GaAs [34]. As previously discussed in section 3.3, the TDLDA result is close to the RPA curve, and both show the well known discrepancies with experiment: the peak positions are redshifted, and the intensity of the first main structure is strongly underestimated. The dashed curve (GW-RPA) is obtained by replacing the Kohn-Sham eigenvalues with GW quasiparticle energies in the RPA calculation 


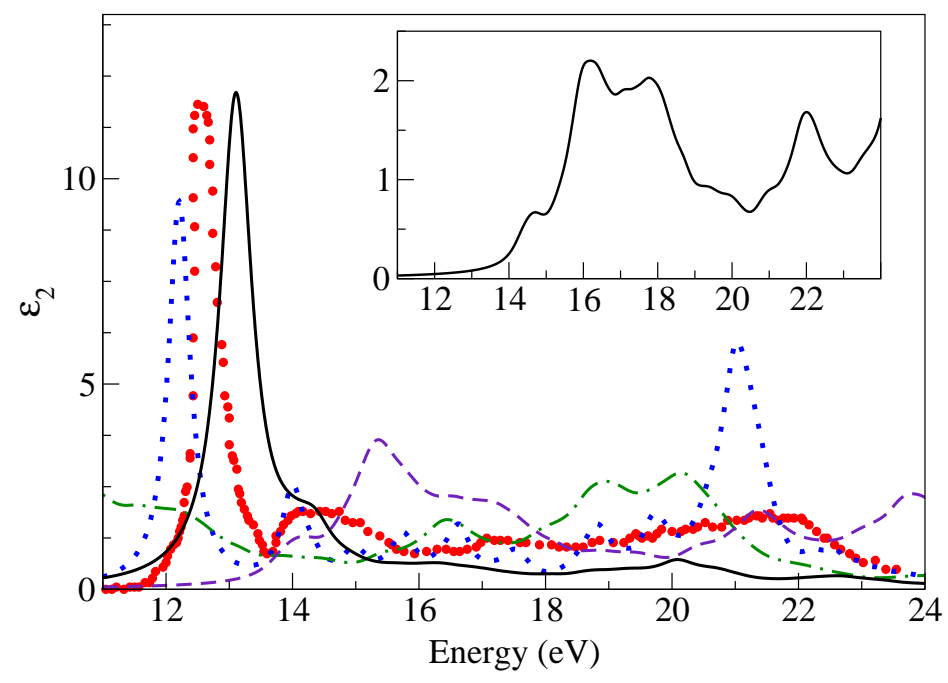

Figure 16. Imaginary part of the macroscopic dielectric function for LiF [87]. Dots: experiment [88]; dotted curve: BSE; dot-dashed curve: TDLDA; dashed curve: TDDFT using the static LRC model kernel; solid line: TDDFT using the dynamical LRC model kernel. In the inset: GW-RPA.

of $\epsilon$. This corresponds to applying the Dyson equation (2.35) for the first part of the exchange-correlation kernel $f_{\mathrm{xc}}^{(1)}$. The resulting spectrum is blueshifted and, moreover, the lineshape has not been corrected. Finally, the curve representing a TDDFT calculation starting from GW quasiparticle energies to calculate the independent-particle response and using the LRC kernel (8.1) gives an excellent fit to the experiment. This curve was obtained using $\alpha^{\text {static }}=0.2$.

The simple static LRC model, together with the linear dependence of $\alpha$ on $1 / \epsilon_{\infty}$, allows one to predict the absorption spectrum from the knowledge of the experimental dielectric constant of the material in question. However, the model $f_{\mathrm{xc}}^{\text {static }}$ of (8.1) has clear limits that become more and more evident as the band gap increases.

For example, already in diamond $[34,154]$ the first shoulder and the main peak in the spectrum cannot be both described with good precision using a single parameter $\alpha^{\text {static }}$. The problem gets even more serious when bound exciton peaks appear in the spectrum, as e.g. for LiF [87] (see figure 16) or solid argon [175]. In fact, the only possible action of the LRC exchange-correlation kernel is to redistribute oscillator strength. In contrast to the full BSE kernel, this remains true also in cases where poles should appear in the bandgap, like, e.g., in $\mathrm{LiF}$ where bound excitons occur in the experimental spectrum (see figure 16). Furthermore, approximations as outlined above are based on a limited range of transitions. When the spectral range gets larger, e.g. when response beyond the absorption region is considered, as a consequence of the change of the limited range of transitions also $f_{\mathrm{xc}}$ has to change. In the case of the model kernel (8.1) this introduces an effective $\omega$-dependence of the parameter $\alpha^{\text {static }}$. In order to describe the plasmon of silicon one could for example still use (8.1), but with an $\alpha^{\text {static }}$ that is an order of magnitude larger than the one that yields a good optical spectrum [34]. This will be further discussed below, after a brief summary of other approaches leading to a LRC.

The simple static LRC model (8.1) does not in fact represent the first attempt to account 
for long-range effects in semiconductors. Already in 1994 Godby and Sham [176] pointed out that long-range density variations give rise to an effective exchange-correlation field. It was then proved [23] that the origin of this exchange-correlation field lies in the macroscopic polarisation. Alternatively, one could avoid the use of the macroscopic polarisation by introducing a scissor-operator quasiparticle correction to the Kohn-Sham gap. The LRC stemming from this discussion has hence the task of increasing the gap (like $f_{\mathrm{xc}}^{(1)}$ ), and has, consequently, a positive sign.

Aulbur, Jönsson and Wilkins [177] studied the problem of determining static dielectric constants. They related an effective exchange-correlation field to the difference between the true and the Kohn-Sham static susceptibilities, and, by using calculated Kohn-Sham and measured (i.e., "true") values, fixed the prefactor of a LRC to the kernel for a series of materials. It resulted a contribution $\Delta f_{x c}=\gamma / q^{2}$, where $\gamma$ is of the order of 0.25 for several small-, and medium-gap semiconductors. According to this work, the LRC correction should account for both $f_{\mathrm{xc}}^{(1)}$ and $f_{\mathrm{xc}}^{(2)}$, which is the reason why $\gamma$ turns out to be small and positive. However, this model can not be extended to the description of spectra, as the quasiparticle corrections simulated by $f_{\mathrm{xc}}^{(1)}$ are too complex to be written as a simple LRC model.

Another approach to the description of the dynamical susceptibility was proposed by Boeij et al [158] who obtained a polarisation-dependent functional derived from current density functional theory [178] (see section 7). This functional involves two parameters: one (material-dependent) accounting for a positive shift of transition energies (in fact, a scissor operator), and a second (constant) one, chosen to be 0.4 , that multiplies a tensor $\boldsymbol{Y}$ containing the polarisation effects. The tensor $\boldsymbol{Y}$ is in principle frequency-dependent, but its static value is used. This approach yields identical results to the static LRC model described here, if one identifies $0.4 Y=-\alpha^{\text {static }}$, and if the scissors are replaced by the quasiparticle corrections.

In consideration of the limits of the static LRC model, it is natural trying to guess how to further improve the kernel. One can either work along the lines of [29] and add a more complicated spatial behaviour, or one can keep the simple $1 / q^{2}$ form of the LRC, but introduce a frequency dependent $\alpha$ [154]. Clearly, this latter choice allows for each structure of the spectrum to have its own effective correction. Botti et al [87] proposed a frequency dependent LRC kernel of the form

$$
f_{\mathrm{xc}}^{\mathrm{dyn}}(\mathbf{q}, \omega)=-\frac{1}{q^{2}}\left(\alpha+\beta \omega^{2}\right) .
$$

This choice was guided by recent calculations [154] for bulk silicon and diamond, that yielded the frequency dependence of the LRC term of the exchange-correlation kernel from the inversion of the BSE. Figure 17 shows, for $f_{\mathrm{xc}}$ of silicon, that the dynamical model $f_{\mathrm{xc}}^{\mathrm{dyn}}$ is indeed a good approximation in a large energy range, even including the plasmon region (see inset figure 17).

Starting from (6.20) it is possible to prove that the two parameters $\alpha$ and $\beta$ of (8.2) can be related to physical quantities, namely the dielectric constant and the plasma frequency. For simple semiconductors, this model yields the same result as the static LRC model [87]. In the case of wide-gap insulators, like diamond or cadmium selenide, or for the EELS of silicon, the dynamical model heals the shortcomings of the static LRC kernel [87]. The improvement is significant even in the presence of bound excitons. We consider again as an example $\mathrm{LiF}$ (see figure 16). Setting $\alpha^{\text {static }}$ to the value 2.0 gives a reasonable compromise (dashed line), enhancing slightly the low energy structures without provoking the collapse of the spectrum. The worst disagreement concerns the absence of the large excitonic peak at about 


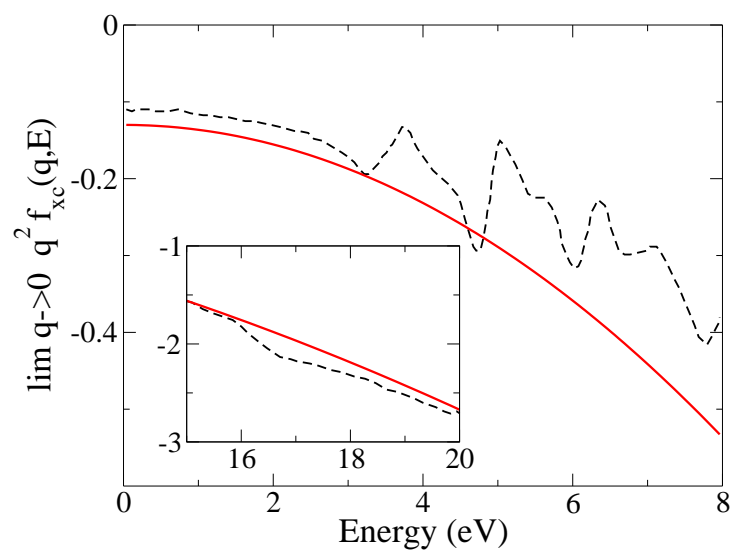

Figure 17. Real part of the LRC component of the exchange-correlation kernel needed to reproduce the BSE optical spectrum of silicon. The inset shows the same function in an energy region close to the plasma frequency. Dashed lines: calculation from [154]; solid line: dynamical LRC model.

$12.5 \mathrm{eV}$. On the other hand, the dynamical model (solid line) is able to describe the strong bound exciton peak. A better agreement can only be found using the BSE approach (dotted line) or the full kernel derived by the BSE (see figure 12), both of which involve a much larger computational effort $[89,179,91,92]$ than the application of the simple dynamical LRC model. Note that the objective of these model kernels is not to provide an ab initio approximation to the exact TDDFT kernel, but to offer a numerically efficient framework for the calculation of response properties of complex solids and nanostructures. A key feature is that the model kernels depend on a reduced number of parameters, and that these can be related in a straightforward manner to known physical quantities. The drawback of these models is, of course, the reduced range of validity of the underlying approximation, which implies that one should carefully check the applicability of a model to a specific system.

\subsection{Contact exciton}

The contact exciton model was successfully used in the 70 s for the description of continuum exciton effects in a wide range of systems [180, 181, 182, 183]. Model calculations could also show that the contact exciton is able to produce one bound state $[184,183]$. This simple model gives rise to the equation

$$
\epsilon_{\mathrm{M}}(\omega)-1=\frac{\epsilon_{\mathrm{M}}^{\mathrm{RPA}}(\omega)-1}{1+g\left(\epsilon_{\mathrm{M}}^{\mathrm{RPA}}(\omega)-1\right)} .
$$

Inspection reveals that this is the LRC kernel approximation derived in the previous section $[154,175]$, provided that $f_{\mathrm{xc}}=-g 4 \pi / q^{2}$. Moreover, in [180] the parameter $g$ is dependent on $\omega^{2}$, similarly to the parameter $\beta$ of the dynamical LRC model of section 8.1.

A different exchange-correlation kernel can be derived in the same spirit by replacing the screened electron-hole interaction in the BSE by a local contact potential [175]. When $W$ in (6.14) is approximated by $\delta(12) A$, the equality is possible in (6.15) and one obtains

$$
f_{\mathrm{xc}}^{\text {contact }}\left(\mathbf{r}_{1}, \mathbf{r}_{3}\right)=-\frac{1}{2} \delta\left(\mathbf{r}_{1}-\mathbf{r}_{3}\right) A \text {. }
$$




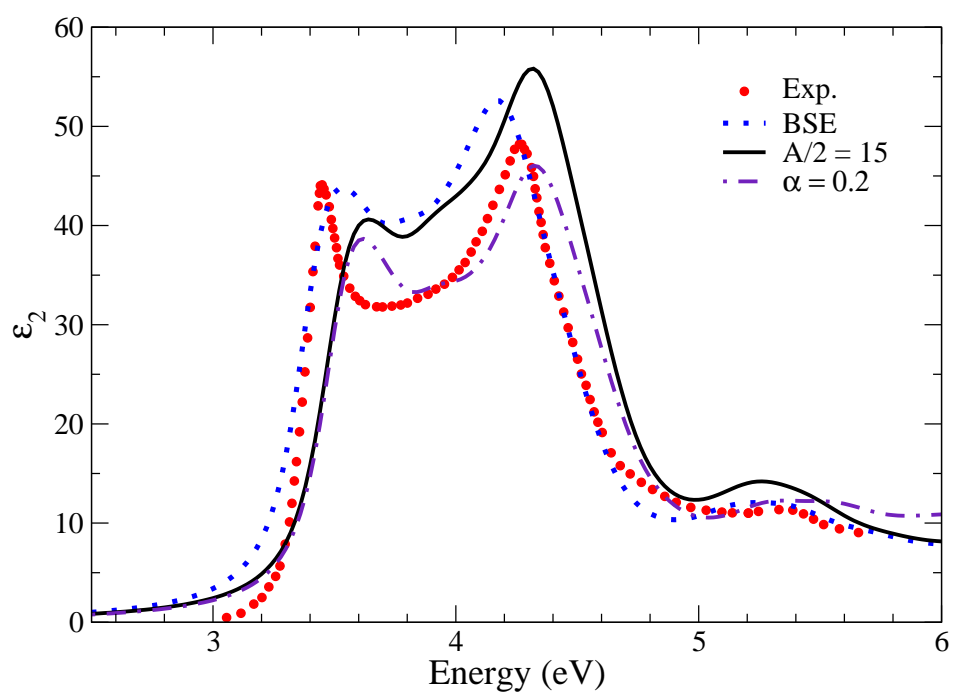

Figure 18. Imaginary part of the macroscopic dielectric function for bulk silicon. Dotted curve: BSE; Dot-dashed curve: TDDFT with static LRC $\left(\alpha^{\text {static }}=0.2\right.$; Continuous curve: TDDFT contact kernel $(A / 2=15)$; Dots: experiment [93].

This is obviously a ultra-short range kernel.

In figure 18 we compare optical absorption calculations performend within the different approximations with the experimental optical spectrum of silicon. The curve obtained for the ultra-short ranged $f_{x c}^{\text {contact }}$ using $A / 2=15$ (continuous curve) turns out to be in very good agreement with the result of a full BSE calculation (dotted curve) and the long-range kernel $f_{\mathrm{xc}}^{\text {static }}=-\alpha / q^{2}$ with $\alpha=0.2$ (dot-dashed curve). All calculations are also in good agreement with experiment (circles).

In order to understand how a short-range and a long-range $f_{\mathrm{xc}}$ can yield similar spectra for simple semiconductors, one has to realise that we are talking about effective exchangecorrelation kernels, and not the real one. These kernels are chosen to reproduce a certain number of properties. It is clear that more than one kernel (even with very different analytical forms and physical interpretation) can lead to the same optical spectrum. The contact exciton is a very good illustration of this.

To a certain extent, a short-ranged but strong electron-hole Coulomb interaction can simulate the effect of the true, screened long-ranged one: continuum exciton effects can be well reproduced, and even one bound exciton can be created. However, in the latter case the continuum is not well described, and no higher-order peaks of the series are obtained.

The choice of which model to use should finally depend both on computational convenience and on the possibility to determine the parameters of the model without fitting to experiment. The stronger are the requirements on the precision of the results, the closer the chosen model should resemble to the exact $f_{\mathrm{xc}}$ and, for example, have a long range component when the system is a semiconductor or insulator. We remark that all these methods discussed above are the result of quite recent investigations, so it is very reasonable to expect further developments in the near future. 


\section{Conclusions}

After the pioneering work of Zangwill and Soven [15], time dependent density functional theory (TDDFT) found a rigorous foundation in the work by Runge and Gross [16]. The time-dependent Kohn Sham equations are obtained as a generalisation of the static case and, from these, the response functions describing the neutral excitations of the system. The main ingredient is the time dependent exchange-correlation potential $v_{\mathrm{xc}}[n](\mathbf{r}, t)$, that depends on the density at all points in space, and at all past times.

Staying within linear response theory, one needs to know, in addition to the static exchange-correlation (xc) potential $v_{\mathrm{xc}}\left[n_{\mathrm{GS}}\right](\mathbf{r})$, the so called xc kernel $f_{\mathrm{xc}}[n]\left(\mathbf{r}, \mathbf{r}^{\prime}, t, t^{\prime}\right)=$ $\delta v_{\mathrm{xc}}[n](\mathbf{r}, t) / \delta n\left(\mathbf{r}^{\prime}, t^{\prime}\right)$. If these two quantities are known, TDDFT is an exact theory, yielding the exact linear response. The problem is, therefore, how to generate suitable approximations for the potential and the kernel. For finite systems, it is sometimes crucial to have a good $v_{\mathrm{xc}}$, whereras in extended systems in general the main problem is to find a good approximation for the kernel $f_{\mathrm{xc}}$.

Neutral excitations can also be calculated in the framework of Many-Body Perturbation Theory, via the solution of the Bethe-Salpeter equation (BSE). However, in that case one has to deal with two-particle Green's functions, that are four-point quantities, whereas TDDFT is based on two-point linear response functions. Therefore TDDFT promises to be computationally much more efficient, which motivates the search for good approximations.

The simplest approximation is the adiabatic LDA (TDLDA), which often yields good results for finite systems. Its main shortcoming is to miss the long range part, proportional to $1 /\left|\mathbf{r}-\mathbf{r}^{\prime}\right|$, which may be important in extended systems. Nevertheless, the TDLDA describes well also some properties of extended systems, in particular the plasmon structures in the loss function or the dynamical structure factor. In these cases, indeed, the long range part of the kernel is not important, either because of cancellations or because of non-vanishing momentum transfer. However, TDLDA does not describe well the optical properties of extended systems, where the long range part is essential.

Therefore, one of the main challenges of recent years has been to find suitable approximations to the long range part of $f_{\mathrm{xc}}$. Promising solutions came from a comparison of TDDFT and the many-body perturbation theory (MBPT) equations for the polarisation. Different groups have suggested different approaches; all approaches have led to one and the same kernel, the "nanoquanta" kernel, that is linear in the screened Coulomb interaction and based on quasi-particle ingredients. This kernel allows one to obtain a good description of the optical properties of extended systems, including bound excitons. It also describes well spectra for non-vanishing momentum transfer, and it has been demonstrated that it yields very good results for low-dimensional systens, including clusters, wires and surfaces.

At present, although the TDDFT linear response equation is relatively quick to solve because of its two-point nature, calculations involving the "nanoquanta" kernel are still computer-time intensive. In particular, for the calculation of the kernel itself current implementations use ingredients of the BSE approach, so that the performance of the two methods is comparable. However, the current optimization of algorithms and computer codes should lead to considerable speedup. Moreover it has been shown that the new kernel is a convenient starting point for additional approximations, such as the $\alpha / q^{2}$ method described in section 8.1. Hence, we believe that TDDFT using the many-body kernel will be one of the main methods of the future for calculating the optical properties of extended systems. 


\section{Acknowledgments}

This work was funded in part by the EU's 6th Framework Programme through the Nanoquanta Network of Excellence (NMP-4-CT-2004-500198). We acknowledge collaborations with S. Kurth, A. Marini, V. Olevano, A. Rubio, M. Scheffler, F. Sottile, K. Tatarczyk and U. von Barth. We thank I. G. Gurtubay, A. Marini, G. Mulas, D. Varsano and N. Vast for providing their figures included in this paper. We thank M. Marques for the critical reading of the manuscript.

\section{References}

[1] Kohn W 1999 Rev. Mod. Phys. 71 1253-66

2] Hohenberg P and Kohn W 1964 Phys. Rev. 136 B864-71

[3] Kohn W and Sham L J 1965 Phys. Rev. 140 A1133-8

[4] Perdew J P, Burke K and Ernzerhof M 1996 Phys. Rev. Lett. 77 3865-8

[5] Perdew J P, Burke K and Ernzerhof M 1997 Phys. Rev. Lett. 78 1396(E)

[6] Jones R O and Gunnarsson O 1989 Rev. Mod. Phys. 61 689-746

[7] Baroni S and Resta R 1986 Phys. Rev. B 33 7017-21

[8] Kotliar G, Savrasov S Y, Haule K, Oudovenko V S, Parcollet O and Marianetti C A 2006 Rev. Mod. Phys. 78 865-951

[9] Sim F, St-Amand A, Papai I and Salahub D R 1992 J. Am. Chem. Soc. 114 4391-400

[10] Godby R W, Schlüter M and Sham L J 1987 Phys. Rev. B 36 6497-500

[11] Fetter A L and Walecka J D 1971 Quantum Theory of Many-Particle Systems (McGraw-Hill, New York)

[12] Sham L J and Rice T M 1966 Phys. Rev. 144 708-14

[13] Hanke W 1978 Adv. Phys. 27 287-341

[14] Onida G, Reining L and Rubio A 2002 Rev. Mod. Phys. 74 601-59 and refereces therein

[15] Zangwill A and Soven P 1980 Phys. Rev. Lett. 45 204-7

[16] Runge E and Gross E K U 1984 Phys. Rev. Lett. 52 997-1000

[17] Gross E K U and Kohn W 1985 Phys. Rev. Lett. 55 2850-2

[18] Gross E K U and Kohn W 1986 Phys. Rev. Lett. 57 923(E)

[19] van Leeuwen R 2001 Int. J. Mod. Phys. B 15 1969-2023

[20] Petersilka M, Gossmann U J and Gross E K U 1996 Phys. Rev. Lett. 76 1212-5

[21] Marques M A L and Gross E K U 2004 Annu. Rev. Phys. Chem. 55 427-55

[22] Petersilka M, Gross E K U and Burke K 2000 Int. J. Quantum Chem. 80 534-54

[23] Gonze X, Ghosez P, and Godby R W 1995 Phys. Rev. Lett. 74 4035-8

[24] van Gisbergen S J A, Kootstra F, Schipper P R T, Gritsenko O V, Snijders J G and Baerends E J 1999 Phys. Rev. Lett. 83 694-7

[25] Maddocks N E, Godby R W and Needs R J 1994 Europhys. Lett. 27681

[26] Gurtubay I G, Ku W, Pitarke J M, Eguiluz A G, Larson B C, Tischler J and Zschack P 2005 Phys. Rev. B $\mathbf{7 2} 125117$

[27] Waidmann S, Knupfer M, Arnold B, Fink J, Fleszar A and Hanke W 2000 Phys. Rev. B 61 10149-53

[28] Weissker H C, Serrano J, Huotari S, Bruneval F, Sottile F, Monaco G, Krisch M, Olevano V and Reining L Phys. Rev. Lett., to be published

[29] Reining L, Olevano V, Rubio A and Onida G 2002 Phys. Rev. Lett. 88066404

[30] Sottile F, Olevano V and Reining L 2003 Phys. Rev. Lett. 91056402

[31] Adragna G, Del Sole R and Marini A 2003 Phys. Rev. B 68165108

[32] Marini A, Sole R D and Rubio A 2003 Phys. Rev. Lett. 91256402

[33] Stubner R, Tokatly I V and Pankratov O 2004 Phys. Rev. B 70245119

[34] Botti S, Sottile F, Vast N, Olevano V, Weissker H C, Reining L, Onida G, Rubio A, Sole R D and Godby R W 2004 Phys. Rev. B 69155112

[35] von Barth U, Dahlen N E, van Leeuwen R and Stefanucci G 2005 Phys. Rev. B 72235109

[36] Ankudinov A L, Nesvizhskii A I and Rehr J J 2003 Phys. Rev. B 67115120

[37] Kootstra F, de Boeji P L, Leeuwen R and Snijders J G 2002 (Singapore: World Scientific) pp 1155-85

[38] Marques M A L, Ullrich C A, Nogueira F, Rubio A, Burke K and Gross E K U, eds 2006 TimeDependent Density Functional Theory Lecture Notes in Physics (Heidelberg: Springer)

[39] Nunes R W and Vanderbilt D 1994 Phys. Rev. Lett. 73 712-5

[40] Maitra N T and Burke K 2001 Phys. Rev. A 63042501

[41] Maitra N T and Burke K 2001 Phys. Rev. A $64039901(\mathrm{E})$ 
[42] van Leeuwen R 1999 Phys. Rev. Lett. 82 3863-6

[43] Levy M 1982 Phys. Rev. A 26 1200-8

[44] Lieb E H 1983 Int. J. Quantum Chem. 24 243-77

[45] van Leeuwen R 1998 Phys. Rev. Lett. 80 1280-3

[46] Gross E K U, Ullrich C A and Grossman U J 1995 Density Functional Theory vol 337 of NATO ASI Ser. $B$ (New York: Plenum Press) pp 149-71

[47] Gross E K U, Dobson F J and Petersilka M 1996 Topics in Current Chemistry vol 181 (Heidelberg: Springer-Verlag) p 81 r.f. nalewajski ed

[48] Keldysh L V 1965 Sov. Phys. JETP $201018-26$

[49] Ullrich C A, Gossmann U and Gross E K U 1995 Phys. Rev. Lett. 74 872-5

[50] Görling A 1997 Phys. Rev. A 55 2630-9

[51] http://theory.polytechnique.fr/codes/dp.

[52] http://www.fisica.uniroma2.it/ self.

[53] http://exciting.sourceforge.net/.

[54] Bertsch G F, Iwata J I, Rubio A and Yabana K 2000 Phys. Rev. B 62 7998-8002

[55] Castro A, Marques M A L and Rubio A 2004 J. Chem. Phys. 121 3425-3433

[56] Ghosh S K and Dhara A K 1988 Phys. Rev. A 38 1149-58

[57] Kootstra F, de Boeji P L and Snijders J G 2000 J. Chem. Phys. 112 6517-6531

[58] Walker B, Saitta A M, Gebauer R and Baroni S 2006 Phys. Rev. Lett. 96113001

[59] Gunnarsson O and Lundqvist B I 1976 Phys. Rev. B 13 4274-98

[60] Gunnarsson O and Lundqvist B I 1977 Phys. Rev. B 15 6006(E)

[61] Perdew J P and Levy M 1985 Phys. Rev. B 31 6264-72

[62] Ehrenreich H and Cohen M 1959 Phys. Rev. 115 786-90

[63] Adler S L 1962 Phys. Rev. 126 413-20

[64] Wiser N 1963 Phys. Rev. 129 62-9

[65] Del Sole R and Fiorino E 1984 Phys. Rev. B 29 4631-45

[66] Sottile F, Bruneval F, Marinopoulos A G, Dash L K, Botti S, Olevano V, Vast N, Rubio A and Reining L 2005 Int. J. Quantum Chem. 102 684-701

[67] Marques M A L, Castro A and Rubio A 2001 J. Chem. Phys. 115 3006-14

[68] Perdew J P and Kurth S 2003 (Springer-Verlag) pp 1-51

[69] Hybertsen M S and Louie S G 1986 Phys. Rev. B 34 5390-413

[70] Lindhard J 1954 K. Dan. Vidensk. Selsk. Mat. Fys. Medd. 28(8) 57

[71] Perdew J P and Burke K 1996 Int. J. Quantum Chem. 57309

[72] Perdew J P, Kurth S, Zupan A and Blaha P 1999 Phys. Rev. Lett. 82 2544-7

[73] Perdew J P, Kurth S, Zupan A and Blaha P 1999 Phys. Rev. Lett. 82 (E) 5179

[74] Castro A, Marques M A L, Alonso J A and Rubio A 2004 J. Comp. Theoret. Nanoscience 1231-55

[75] Gurtubay I G, Pitarke J M, Ku W, Eguiluz A G, Larson B C, Tischler J, Zschack P and Finkelstein K D 2004 Phys. Rev. B $\mathbf{7 0} 201201(\mathrm{R})$

[76] Ando T 1977 Z. Phys. B 26 263-334

[77] Yabana K and Bertsch G F 1999 Int. J. Quantum Chem. 75 55-66

[78] Onida G, Schmidt W G, Pulci O, Palummo M, Marini A, Hogan C and Del Sole R 2001 Phys. Status Solidi (a) $\mathbf{1 8 8} 1261-6$

[79] Marques M A L, López X, Varsano D, Castro A and Rubio A 2003 Phys. Rev. Lett. 90258101

[80] Marques M A L and Botti S 2005 J. Chem. Phys. 123014310

[81] Rappoport D and Furchte F 2006 vol 706 of Lecture Notes in Physics (Berlin, Heidelberg: Springer) chap 23, pp 337-54

[82] Wang C R C, Pollack S, Cameron D and Kappes M M 1990 J. Chem. Phys. 93 3787-801

[83] Malloci G, Mulas G and Joblin C 2004 A \& A 426 105-117

[84] Joblin C, Léger A and Martin P 1992 ApJL 393 L79

[85] Champagne B, Perpète E A, van Gisbergen S J A, Baerends E J, Snijders J G, Soubra-Ghaoui C, Robins K A and Kirtman B 1998 J. Chem. Phys. 109 10489-98

[86] Gritsenko O V and Baerends E J 2001 Phys. Rev. A 64042506

[87] Botti S, Fourreau A, Nguyen F, Renault Y O, Sottile F and Reining L 2005 Phys. Rev. B 72125203

[88] Roessler D M and Walker W C 1967 J. Opt. Soc. Am. 57 835-6

[89] Albrecht S, Reining L, Del Sole R and Onida G 1998 Phys. Rev. Lett. 80 4510-3

[90] Benedict L X, Shirley E L and Bohn R B 1998 Phys. Rev. Lett. 80 4514-7

[91] Rohlfing M and Louie S G 1998 Phys. Rev. Lett. 81 2312-2315

[92] Rohlfing M and Louie S G 2000 Phys. Rev. B 62 4927-44

[93] Lautenschlager P, Garriga M, Viña L and Cardona M 1987 Phys. Rev. B 36 4821-30

[94] Stiebling J 1978 Z. Phys. B 31 355-7

[95] Olevano V and Reining L 2001 Phys. Rev. Lett. 86 5962-5 
[96] Marinopoulos A, Reining L, Olevano V, Rubio A, Pichler T, Liu X, Knupfer M and Fink J 2002 Phys. Rev. Lett. 8976402

[97] Vast N, Reining L, Olevano V, Schattschneider P and Jouffrey B 2002 Phys. Rev. Lett. 88037601

[98] Dash L K, Vast N, Baranek P, Cheynet M C and Reining L 2004 Phys. Rev. B 70245116

[99] van Leeuwen R and Baerends E J 1994 Phys. Rev. A 49 2421-31

[100] Dobson J F, Bünner M J and Gross E K U 1997 Phys. Rev. Lett. 79 1905-8

101] Tokatly I V 2006 vol 706 of Lecures Notes in Physics (Berlin, Heidelberg: Springer) chap 8, pp 123-36

[102] Maitra N T 2005 J. Chem. Phys. 122 234104-9

[103] Maitra N T, Zhang F, Cave R J and Burke K 2004 J. Chem. Phys. 120 5932-7

[104] Cave R J, Zhang F, Maitra N T and Burke K 2004 Chem. Phys. Lett. 389 39-42

[105] Casida M E 2005 J. Chem. Phys. 122 054111-9

106] Tozer D J and Handy N C 2000 Phys. Chem. Chem. Phys. $22117-2121$

[107] Hirata S and Head-Gordon M 1999 Chem. Phys. Lett. 302 375-82

[108] Tatarczyk K, Schindlmayr A and Scheffler M 2001 Phys. Rev. B 63235106

[109] Krieger J B, Li Y and Iafrate G J 1992 Phys. Rev. A 45 101-26

[110] Kim Y and Görling A 2002 Phys. Rev. B 6635114

[111] Lein M, Gross E K U and Perdew J P 2000 Phys. Rev. B 61 13431-7

[112] Burke K, Petersilka M and Gross E K U 2002 Recent Advances in Density Functional Methods 3 67-79

[113] Corradini M, Del Sole R, Onida G and Palummo M 1998 Phys. Rev. B 57 14569-71

[114] Moroni S, Ceperley D M and Senatore G 1995 Phys. Rev. Lett. 75 689-92

115] Richardson C F and Ashcroft N W 1994 Phys. Rev. B 50 8170-81

[116] Hubbard J 1957 Proc. R. Soc. London A 243 336-52

[117] Vashishta P and Singwi K S 1972 Phys. Rev. B 6 875-87

[118] Vashishta P and Singwi K S 1972 Phys. Rev. B 6 4883(E)

[119] Utsumi K and Ichimaru S 1980 Phys. Rev. B 22 5203-12

[120] Dobson J F 1994 Phys. Rev. Lett. 732244

[121] Dreizler R M and Gross E K U 1990 Density Functional Theory (Springer Verlag)

[122] Lipparini E, Stringari S and Takayanagi K 1994 J. Phys.: Condens. Matter 6 2025-30

[123] Mermin N D 1970 Phys. Rev. B 1 2362-3

124] Kohn W 1996 Phys. Rev. Lett. 76 3168-71

[125] Palummo M, Onida G, Del Sole R, Corradini M and Reining L 1999 Phys. Rev. B 60 11329-35

[126] Gunnarsson O, Jonson M and Lundqvist B I 1979 Phys. Rev. B 20 3136-64

[127] Olevano V, Palummo M, Onida G and Del Sole R 1999 Phys. Rev. B 60 14224-33

[128] van Leeuwen R 1996 Phys. Rev. Lett. 76 3610-3

[129] Hedin L 1965 Phys. Rev. 139 A796-823

[130] Strinati G 1988 Rivista del Nuovo Cimento 111

[131] Sham L J and Schlüter M 1983 Phys. Rev. Lett. 51 1888-91

[132] Bruneval F, Sottile F, Olevano V and Reining L 2006 J. Chem. Phys. 124144113

133] Kim Y and Görling A 2002 Phys. Rev. Lett. 8996402

[134] Sharp R T and Horton G K 1953 Phys. Rev. 90317

[135] Talman J D and Shadwick W F 1976 Phys. Rev. A 14 36-40

[136] Görling A 1996 Phys. Rev. B 56 7024-9

[137] Görling A 1999 Phys. Rev. B 59 10370(E)

138] Städele M, Moukara M, Majewski J A, Vogl P and Görling A 1999 Phys. Rev. B 59 10031-43

[139] Kotani T 1994 Phys. Rev. B 50 14816-21

[140] Kotani T 1995 Phys. Rev. B 51 13903-4(E)

[141] Kotani T 1995 Phys. Rev. Lett. 74 2989-92

[142] Kotani T and Akai H 1996 Phys. Rev. B 54 16502-14

[143] Städele M, Majewski J A, Vogl P and Görling A 1997 Phys. Rev. Lett. 79 2089-92

[144] Magyar R J, Fleszar A and Gross E K U 2004 Phys. Rev. B 69045111

[145] Perdew J and Levy M 1983 Phys. Rev. Lett. 51 1884-7

[146] Tokatly I V and Pankratov O 2001 Phys. Rev. Lett. 86 2078-81

[147] Brosens F, Devrese J T and Lemmens L F 1980 Phys. Rev. B 21 1363-79

[148] Aulbur W G, Städele M and Görling A 2000 Phys. Rev. B 62 7121-32

149] Grüning M, Marini A and Rubio A 2006 J. Chem. Phys. 124154108

[150] Bruneval F, Sottile F, Olevano V, Del Sole R and Reining L 2005 Phys. Rev. Lett. 94186402

[151] Del Sole R, Reining L and Godby R W 1994 Phys. Rev. B 49 8024-8

[152] Streitenberger P 1884 Phys. Stat. Sol.(B) 125(22) 681-92

[153] Streitenberger P 1884 Phys. Lett. A 106 57-60

[154] Del Sole R, Adragna G, Olevano V and Reining L 2003 Phys. Rev. B 67045207

[155] Casida M E 1995 Recent Advances in Density Functional Methods (Singapore: World Scientific) p 155 
ed. by D. P. Chong

[156] Tokatly I V, Stubner R and Pankratov O 2002 Phys. Rev. B 65113107

[157] Hedin L and Lundqvist S 1969 Solid State Physics vol 23 (Academic, New York) pp 1-181

[158] de Boeij P L, Kootstra F, Berger J A, van Leeuwen R and Snijders G 2001 J. Chem. Phys. 115 1995-9

[159] van Faassen M, de Boeij P L, van Leeuwen R, Berger J A and Snijders J G 2003 J. Chem. Phys. 118 $1044-53$

[160] Marini A, Sole R D and Rubio A 2006 vol 706 of Lecture Notes in Physics (Berlin, Heidelberg: Springer) chap 20, pp 301-16

[161] Varsano D, Marini A and Rubio A 2006 To be published

[162] Maitra N T, Sousa I and Burke K 2003 Phys. Rev. B 68045109

[163] Dhara A K and Ghosh S K 1987 Phys. Rev. A 35 442-4

[164] D'Agosta R and Vignale G 2005 Phys. Rev. B 71245103

[165] Vignale G 2004 Phys. Rev. B 70 201102(R)

[166] Vignale G and Kohn W 1996 Phys. Rev. Lett. 77 2037-40

[167] Vignale G, Ullrich C A and Conti S 1997 Phys. Rev. Lett. 79 4878-81

168] Vignale G 2001 Int. J. Mod. Phys. 15 1714-23

[169] Ullrich C A and Vignale G 2001 Phys. Rev. Lett. 87037402

[170] Ullrich C A and Vignale G 2002 Phys. Rev. B 65245102

[171] Ullrich C A and Vignale G 2004 Phys. Rev. B 70 239903(E)

[172] van Faassen M, de Boeij P L, van Leeuwen R, Berger J A and Snijders J G 2002 Phys. Rev. Lett. 88 186401

[173] Ghosez P, Gonze X and Godby R W 1997 Phys. Rev. B 5612811

[174] Lautenschlager P, Garriga M, Logothetidis S and Cardona M 1987 Phys. Rev. B 35 9174-89

[175] Sottile F, Karlsson K, Reining L and Aryasetiawan F 2003 Phys. Rev. B 68205112

[176] Godby R W and Sham L J 1994 Phys. Rev. B 49 1849-57

[177] Aulbur W G, Jönsson L and Wilkins J W 1996 Phys. Rev. B 54 8540-50

[178] Vignale G and Kohn W 1998 Electronic Density Functional Theory: Recent Progress and New Directions (New York: Plenum) ed. by J. Dobson et al.

[179] Benedict L X, Shirley E L and Bohn R B 1998 Phys. Rev. B 57 R9385-R9387

180] Rowe J and Aspnes D 1970 Phys. Rev. Lett. 25 162-5

[181] Rowe J and Aspnes D 1970 Phys. Rev. Lett. 25979 (E)

[182] Martin R M, van Vechten J A, Rowe J E and Aspnes D E 1972 Phys. Rev. B 6 2500-2

[183] Schwabe N F and Elliott R J 1996 Phys. Rev. B 53 5318-29

[184] Egri I 1985 Physics Report 119 363-402 and references therein. 